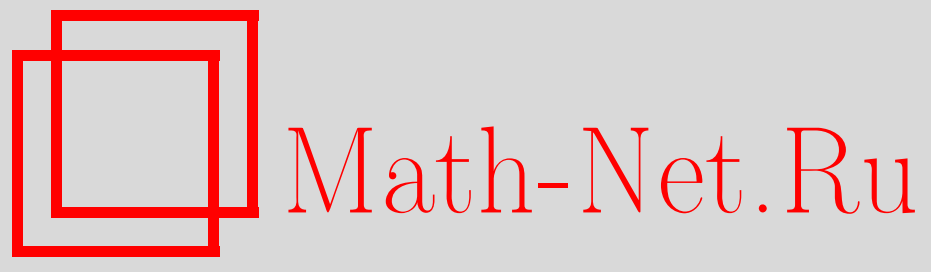

В. Г. Кановей, В. А. Любецкий, Проблемы теоретикомножественного нестандартного анализа, УМH, 2007, том 62, выпуск 1, 51-122

DOI: https://doi.org/10.4213/rm5588

Использование Общероссийского математического портала Math-Net.Ru подразумевает, что вы прочитали и согласны с пользовательским соглашением http://www . mathnet.ru/rus/agreement

Параметры загрузки:

IP : 54.157 .27 .8

26 апреля 2023 г., 14:34:20

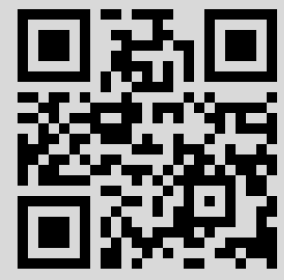


УДК 510.223

\section{Проблемы теоретико-множественного нестандартного анализа}

\section{В. Г. Кановей, В. А. Любецкий}

В статье излагаются главные результаты последних лет в области теоретико-множественного нестандартного анализа (нестандартные теории множеств и классов). Показано, каким образом универсумы более простых теорий (начиная с обычной теории множеств Цермело-Френкеля ZFC) могут быть расширены до универсумов более сложных нестандартных теорий множеств и классов. Заключительный параграф излагает основные положения булевозначного анализа как части теоретико-множественного нестандартного анализа.

Библиография: 66 названий.

\section{СОДЕРЖАНИЕ}

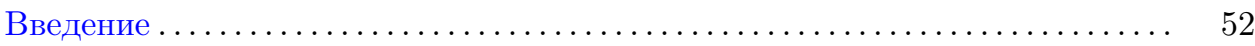

$\S 1$. От стандартного универсума множеств к нестандартному ......... 56

1а. Множества, классы, реляционные структуры .............. 57

1b. Элементарные расширения и прямые пределы .............. 58

1с. Насыщенные структуры и расширения .................. 59

1d. "Эффективные” насыщенные расширения................. 62

1е. Абсолютно насыщенные расширения .................... 64

1f. Теория ограниченных множеств .................... 66

1g. Абсолютно насыщенное расширение стандартного универсума .. 68

1h. Теория ограниченных множеств: приведение формул к $\Sigma_{2}^{\mathrm{st}}$-виду 70

$\S 2$. Присоединение классов к нестандартному универсуму ........... 74

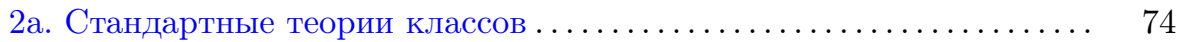

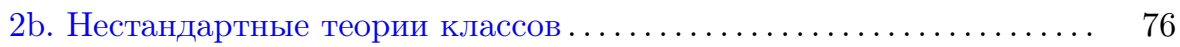

2c. Аксиома непредикативного Переноса в теории Келли-Морса ... . 79

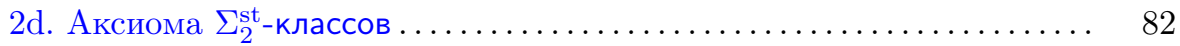

2е. Полумножества и аксиома $\Sigma_{2}^{\text {st }}$-полумножеств .................... 84

2f. Интерпретация нестандартных теорий классов в стандартных ... 86

$2 \mathrm{~g}$. Метаматематические следствия ...................... 90

Первый автор поддержан грантами РФФИ 03-01-00757 и 06-01-00608 и DFG 436 RUS 17/68/05; второй автор поддержан грантами Минпромнауки РФ 37-053-11-0061 и МНТЦ 2766.

(C) В. Г. КАновЕй, В. А. ЛюБЕцКий, 2007 
$\S 3$. Присоединение внешних множеств ........................ 92

3а. Структура "внешнего" универсума теории Хрбачека ........... 92

3b. Нестандартные теории классов на базе теории Хрбачека ........ 94

3с. Кумулятивное построение множеств и классов ............... 96

3d. Кумулятивное расширение нестандартного универсума классов . 101

3е. Свойства кумулятивного расширения .................. 103

3f. Метаматематические следствия . . . . . . . . . . . . . . . . . 106

$3 \mathrm{~g}$. Как избавиться от предположения о существовании оболочки из классов ..................................... 107

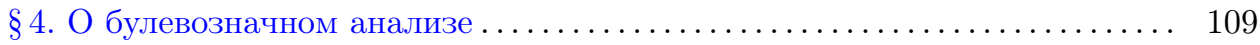

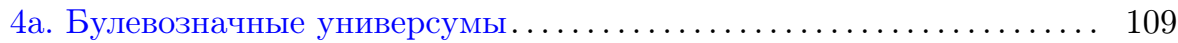

4b. Вложения стандартного универсума в булевозначный . . . . . . . 110

4c. Теоремы переноса для булевозначного универсума............ 111

4d. Нестандартные представления полных метрических пространств 112

4е. Пример: кольца функций . . . . . . . . . . . . . . . . . 115

4f. Нестандартное представление стандартного гомоморфизма групп 117

Список литературы .......................................... 119

\section{Введение}

Современный нестандартный анализ возник в начале 1960-х годов, когда А. Робинсон [1; гл. IX] показал, как можно использовать нестандартные модели алгебраических структур и поля вещественных чисел для получения математических результатов (см. книги В. А. Успенского [2], [3] или книгу Е. И. Гордона, А. Г. Кусраева и С. С. Кутателадзе [4] о предшествующей нестандартному анализу истории бесконечно больших и бесконечно малых величин, восходящей к работам Ньютона, Лейбница и более ранних математиков).

Одна из исходных идей нестандартного анализа состоит в таком представлении (стандартных) математических структур $\mathfrak{A}$ с помощью их (нестандартных) расширений $* \mathfrak{A}$, которое позволяет свести (редуцировать) сложные вопросы о исходной стандартной структуре $\mathfrak{A}$ к простым вопросам о ее нестандартном расширении *A. Один из первых примеров такого сведе́ния был предложен самим Робинсоном практически одновременно с формулировкой нестандартного анализа. Это было решение довольно старой и открытой на тот момент проблемы о существовании инвариантного подпространства у каждого нормального оператора в бесконечномерном пространстве [5; с. 183-222]. Сведе́ние состояло в замене этого сложного вопроса на вопрос, имеющий заранее очевидный ответ: имеется ли инвариантное подпространство у матрицы, действующей в конечномерном пространстве? Это было сделано с помощью понятия о (нестандартном) натуральном числе $h$, превосходящем все стандартные натуральные числа - тогда исходное бесконечномерное пространство представлялось (нестандартным) $h$-мерным векторным пространством. Другие примеры такого рода применений нестандартного анализа можно найти, в частности, в [5], [6], и в более современных публикациях [4], [7].

Уже к концу 1960-х годов стало ясно, что теоретико-модельные методы, использованные Робинсоном, применимы к любой математической структуре $\mathfrak{A}$, и в особенности там, где алгебраические понятия и также расстояние, близость, 
мера играют существенную роль. Одна из форм нестандартного анализа состоит в построении нестандартного расширения ${ }^{*} \mathfrak{A}$ исходной математической структуры $\mathfrak{A}$ вместе с соответствующим вложением $\mathfrak{A} \rightarrow * \mathfrak{A}$, обозначаемым $a{ }^{*} a$ (или $a \mapsto a^{\vee}$ в булевозначном случае). Известно несколько методов построения таких расширений $* \mathfrak{A}$, например, с помощью улътрастепени исходной структуры $\mathfrak{A}$, которое зависит от выбора ультрафильтра $U$ на множестве индексов.

Быстро выяснилось, что применимость нестандартных методов связана не с деталями построения нестандартного расширения ${ }^{*} \mathfrak{A}$, а с небольшим числом общих принципов вроде элементарности и насыщенности. Свойство элементарности состоит в том, что объект $a \in \mathfrak{A}$ имеет в $\mathfrak{A}$ те же свойства, что и объект ${ }^{*} a$ в * $A$. Свойство насыщенности, которое имеет в качестве параметра несчетный кардинал $\kappa$ (зависящий, например, от выбора ультрафильтра $U)$, состоит в том, что любая система $S$ из каких-то условий $P_{\alpha}(x)$ на искомое $x$ в количестве строго меньшем $\kappa$, для которой каждая конечная подсистема $S^{\prime} \subseteq S$ этих условий имеет решение в * $\mathfrak{A}$, имеет общее решение в ${ }^{*} \mathfrak{A}$. Например, $\aleph_{1}$-насыщенность нестандартного расширения $* \mathbb{N}$ натурального ряда $\mathbb{N}$ приводит к существованию бесконечно большого натурального числа $h$ в *N. В результате уже в 1970-е годы возникла аксиоматическая форма нестандартного анализа, т.е. такая, в которой нестандартное расширение *V класса всех множеств V описывается аксиоматически. Началось изучение нестандартных теоретико-множественных аксиоматик типа теории внутренних множеств Нельсона IST [8], [9], нестандартных теорий Хрбачека [10], [11] и Каваи [12], [13]. Это направление, связанное с наиболее фундаментальными проблемами нестандартной математики, называется теоретико-множественнъмм нестандартным анализом в отличие от более частного прикладного (или робинсоновского) нестандартного анализа, занятого изучением нестандартных расширений * $\mathfrak{A}$ отдельных математических структур $\mathfrak{A}$.

Открытые в [14] свойства ограниченных множеств - элементов стандартных множеств, в частности, теорема о приведении формул к $\Sigma_{2}^{\text {st }}$-виду (теорема 1.19 ниже), легли в основу серии глубоких исследований в области теоретико-множественного нестандартного анализа [15]-[24], включая книгу [25].

Уже после выхода монографии [25], в 2004-05 гг. среди специалистов в этой области значительно возрос интерес к нестандартным теориям классов, взаимоотношения которых с соответствующими нестандартными теориями множеств оказались не столь простыми, как в случае отсутствия всяких нестандартностей. В результате удалось получить решения нескольких фундаментальных проблем теоретико-множественного нестандартного анализа, которым, в основном, посвящена эта статья.

ПроБЛЕмА 1. (а) Можно ли построить “эффективно", т.е. с помощью явной конструкции, насыщенные расширения математических структур? ${ }^{1}$

(б) Существуют ли абсолютно насыщенные (т.е. к-насыщенные для каждого кардинала $\kappa$ ) “эффективные” элементарные расширения?

\footnotetext{
1Эта проблема была сообщена одному из авторов настоящей статьи (В. Г. Кановею) В. А. Успенским в начале 1990-х годов.
} 
Интерес к вопросу (а) связан с тем, что традиционная техника построения нестандартного расширения * $\mathfrak{A}$ требует предварительного выбора ультрафильтра $U$ (для ультрапроизведений) или, взамен, например, полного упорядочения области исходной структуры $\mathfrak{A}$ - короче, объектов, существование которых основано на полной аксиоме выбора и которые не могут быть представлены в виде конкретной, "эффективной” конструкции. Аналогия с другими подобными вопросами (например, с вопросом о существовании неизмеримых по Лебегу множеств вещественных чисел [26], [27]) приводила к мысли об отрицательном ответе на этот вопрос. Но ответ оказался положительным (В. Г. Кановей, В. А. Успенский, С. Шелах, [24], [28]).

Даже в отсутствие требования эффективности вопрос (б) представляет большие трудности, связанные с тем, что такие абсолютно насыщенные элементарные расширения должны быть собственными классами и их наиболее естественное построение предполагает предварительный выбор цепочки ультрафильтров длины Ord - для чего нужна аксиома глобального выбора, выходящая за рамки даже всей обычной теории множеств Цермело-Френкеля ZFC. В 11 мы покажем, что и этот вопрос решается положительно: теория ZFC достаточно сильна для построения абсолютно насыщенного расширения всего "стандартного" универсума множеств, и такое расширение удовлетворяет аксиомам известной нестандартной теории множеств BST.

ПроБлемА 2. Как присоединять классы к нестандартному универсуму множеств для построения структур, удовлетворяющих той или иной нестандартной теории классов, предполагая, что стандартная область множеств уже расширена стандартными классами?

Здесь задача состоит в том, чтобы превратить оболочку стандартных классов над стандартной областью всех множеств в оболочку нестандартных классов над нестандартной областью всех множеств. В относительно простом случае предикативных теорий классов это было сделано в [15]. Теперь получено решение и для непредикативных теорий классов. Оно излагается в $\S 2$.

ПроблемА 3. Как присоединять “внешние" множества к нестандартному универсуму множеств "внутреннего" типа, с тем чтобы полученный в результате расширенный универсум удовлетворял разумной “внешней” нестандартной теории множеств?

Нестандартные теоретико-множественные универсумы отличаются от стандартных тем, что кроме отношения принадлежности $\in$ в них рассматривается еще и отношение стандартности st $(\cdot)$. Универсумами "внутреннего" типа называются элементарные (в $\in$-языке) расширения стандартного универсума. Обычно они не удовлетворяют аксиоме выделения в расширенном st- $\in$-языке, т.e. st- $\in$-определимые части множеств не обязательно являются снова множествами. Напротив, нестандартные универсумы "внешнего" типа удовлетворяют аксиоме выделения в st- $\in$-языке - что, конечно, значительно удобнее для приложений, но зато они не являются элементарными расширениями своей стандартной части. (Известны нестандартные теории множеств, описывающие универсумы обоих типов, - о них скажем ниже.) Естественный способ превращения "внутреннего" универсума во "внешний" состоит в присоединении всех 
таких st- $\in$-определимых "не-множеств", затем всех подходящих совокупностей из множеств и "не-множеств" и т. д. В 33 показано, как придать этому процессу расширения точную форму, которая решает проблему 3 на уровне множеств и на уровне классов.

В заключительном параграфе мы вкратце коснемся вопросов, хотя и не относящихся к нестандартному анализу в узком смысле, но связанных с ним методологией и общим местом в математике. В известном смысле речь идет об обобщении подхода и метода нестандартного анализа. Действительно, сущность нестандартных теоретико-множественных универсумов и вообще нестандартных структур состоит в изучении своеобразного инструмента вычисления истинности суждений. Например, в простейшем случае ультрастепени $\mathfrak{A}^{I} / U$, где $\mathfrak{A}$ - некоторая исходная структура и $U$ - некоторый ультрафильтр над индексным множеством $I$, функции из $I$ в $\mathfrak{A}$ составляют универсум нестандартного расширения * $\mathfrak{A}$ структуры $\mathfrak{A}$. При этом исходные элементы из $\mathfrak{A}$ представляются функциями-константами. Для вычисления истинности в * формулы $\varphi\left(f_{1}, \ldots, f_{n}\right)$ (где все $f_{i}$ принадлежат декартовой степени $\mathfrak{A}^{I}$ ) определяется ощенка

$$
\llbracket \varphi \rrbracket=\left\{i \in I: \mathfrak{A} \models \varphi\left(f_{1}(i), \ldots, f_{n}(i)\right)\right\}
$$

со значением в булевой алгебре $\mathscr{P}(I)=\{X: X \subseteq I\}$. Здесь $\mathfrak{A} \models \cdot$ означает "выполняется", "истинно" в $\mathfrak{A}$. По теореме Лося суждение $\varphi$ истинно в * $A$, если и только если его оценка $\llbracket \rrbracket$ принадлежит $U$. Поэтому выяснение вопроса об истинности $* \mathfrak{A} \models \varphi$ сводится к вопросу, чему равна оценка $\llbracket \varphi \rrbracket$, т.е. к более простому понятию $\mathfrak{A}=\varphi$.

Такого рода расширения с оценками можно строить для любой булевой, гейтинговой и даже еще более общих алгебр и решеток В. Этот общий подход включает вместе с нестандартным анализом метод форсинга в современной теории множеств, булевозначный и гейтинговозначный анализы. Вместо факторизации по ультрафильтру истинность свойства $\varphi$ в расширенной структуре

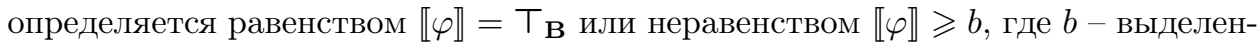
ный элемент из алгебры В. Ценность этого метода состоит в таком представлении математических структур, которое позволяет вместо изучения проблем, непосредственно относящихся к ним, вычислять оценки суждений, связанных с этими проблемами. Примером является упомянутая выше теорема Робинсона.

ПроБЛЕмА 4. Как систематически определять такого рода “упрощающие представления" - переход от обычных структур и отношений к более простым (но связанным с исходными) нестандартным структурам и отношениям? Как строить и классифицировать соответствующие им оценки?

Эта программа действий успешно выполняется начиная с 1960-х годов в рамках нестандартного анализа и с 1970-х годов в рамках булевозначного и гейтинговозначного анализов. Не претендуя на полноту охвата этой проблемы (ей посвящены, например, публикации [5], [29]-[33] на русском языке), мы приведем в $\S 4$ некоторые интересные результаты в этом направлении. 


\section{Общие обозначения.}

$$
\begin{aligned}
& \operatorname{dom} f \quad \text { - область определения функции } f \text {, } \\
& \operatorname{dom} X=\{x: \exists y(\langle x, y\rangle \in X)\} \text { для любого бинарного отношения } X \text {; } \\
& \operatorname{ran} f \quad \text { - область значений функции } f \text {; } \\
& \text { вложение - (или инъекция) } X \text { в } Y \text { - это взаимно однозначное } \\
& \text { отображение между } X \text { и частью } Y \text {; } \\
& f " X=\{f(x): x \in \operatorname{dom} f \cap X\}, f \text {-образ множества } X \text {; } \\
& \operatorname{card} X-\text { мощность множества } X \text {; } \\
& \text { Card - класс всех кардиналов; } \\
& \text { Ord - класс всех ординалов; } \\
& \mathscr{P}(X) \quad=\{Y: Y \subseteq X\}, \text { совокупность всех подмножеств множества } X \text {; } \\
& \mathbf{V}_{\alpha} \quad-\alpha \text {-й уровень кумулятивной иерархии фон Неймана; } \\
& \text { напомним, что } \mathbf{V}_{0}=\varnothing \text { и далее по трансфинитной индукции } \\
& \mathbf{V}_{\alpha}=\mathscr{P}\left(\bigcup_{\gamma<\alpha} \mathbf{V}_{\gamma}\right) \text {, a } \mathbf{V}=\bigcup_{\alpha \in \text { Ord }} \mathbf{V}_{\alpha}- \\
& \text { универсум всех множеств в ZFC; } \\
& \operatorname{rk} x=\min \left\{\alpha \in \text { Ord }: x \in \mathbf{V}_{\alpha}\right\} \text {, ранг множества } x \text { по фон Нейману. }
\end{aligned}
$$

Нашей базовой теорией является теория множеств Цермело-Френкеля ZFC (с аксиомой выбора). Все случаи отклонения от этого соглашения (например, использование теории классов или нестандартной теории) будут оговариваться. Ссылки по теории множеств - это [34]-[38]. Общая ссылка по теории моделей это [39]. Ссылки по "робинсоновскому", или теоретико-модельному, нестандартному анализу - это [40]-[46] и на русском языке [5], [47], [29], [32], [2], [3]. Общей ссылкой по теоретико-множественному нестандартному анализу является книга [25].

Изложение в настоящей статье строится так, чтобы быть понятным любому математику, знакомому с основами теории множеств и нестандартного анализа.

\section{$\S 1$. От стандартного универсума множеств к нестандартному}

Этот параграф посвящен проблеме 1 из введения: эффективное построение насыщенных элементарных расширений исходных, "стандартных" математических структур (включая и наиболее универсальную из них - теоретико-множественный универсум), а также, на этой основе, построение абсолютно насыщенных элементарных расширений. Мы начинаем (четыре первых пункта) с компактного изложения некоторых понятий и результатов теории моделей, необходимых для понимания более существенных результатов из последующих разделов.

Ключевое построение "эффективного" насыщения данной $\in$-структуры по [24], [28] изложено в п. 1d. Оно используется для построения эффективных и абсолютно насыщенных расширений в п. 1е. Затем мы доказываем в п. 1g, 
что такое расширение всего универсума ZFC удовлетворяет аксиомам теории внутренних множеств BST - одной из нестандартных теорий множеств, которая вкратце рассматривается в п. 1f. Мы заканчиваем в п. 1h важной теоремой о приведении формул к $\Sigma_{2}^{\text {st }}$-виду в BST, на которой в значительной степени основывается последующее изложение.

1а. Множества, классы, реляционные структуры. Специфика рассматриваемых в этой статье вопросов такова, что в них фигурируют не только множества, но и классы. Рассуждая в ZFC, под классом понимают любую совокупность множеств, определенную формулой в виде $\left\{x: \varphi\left(x, p_{1}, \ldots, p_{n}\right)\right\}$, где $p_{1}, \ldots, p_{n}$ - множества, называемые параметрами определения. Некоторые совокупности такого вида будут множествами, например, $\{x: \varphi(x) \wedge x \in y\}-$ по аксиоме Выделения. Те, которые не являются множествами, например класс $\mathbf{V}=\{x: x=x\}$ всех множеств, называются собственными классами.

Это, конечно, неформальное понимание, однако оказывается, что достаточно простые и разумные утверждения о классах в этом смысле допускают естественную трансформацию к “законным" утверждениям о множествах. Например, утверждение $\forall X \exists Y \Phi(X, Y)$ ( $X, Y$ - классы) адекватно понимается как тот факт, что по любой формуле $\varphi$ можно указать формулу $\psi$ таким образом, что имеет место $\Phi(X, Y)$, где $X, Y$ - классы, определенные формулами $\varphi, \psi$ соответственно.

С другой стороны, для работы с классами можно использовать теорию классов Гёделя-Бернайса GB (см. п. 2а), где классы являются формальными объектами (а множества - это те классы, которые являются элементами других классов). Здесь важно то, что теория $\mathbf{G B}$ - консервативное расширение ZFC в том смысле, что любая теорема GB, говорящая только о множествах, является и теоремой $\mathbf{Z F C .}$

Вообще, все, что делается ниже с классами в универсуме множеств ZFC, совместимо как с первым (неформальным), так и со вторым (GB) подходом.

Мы будем рассматривать реляционнъе структуръь ${ }^{2}$ вида $\mathfrak{A}=\left\langle A ; R_{1}, \ldots, R_{n}\right\rangle$, где $A=|\mathfrak{A}|$ - множество или класс, называемое универсумом структуры и часто отождествляемое с самой $\mathfrak{A}$ (через запись типа $A=\left\langle A ; R_{1}, \ldots, R_{n}\right\rangle$ ), a $R_{1}, \ldots, R_{n}$ - конечный список финитарных отношений на $A$. Например, структура натуральных чисел может быть представлена в виде $\mathbb{N}=\langle\mathbb{N} ;+, \times, 0,1\rangle$, где $\mathbb{N}=$ $\{0,1,2, \ldots\}$ - само множество натуральных чисел, + обозначает тернарное отношение $x+y=z, \times$ понимается аналогично, а 0 и 1 - константы, естественно представляемые как унарные отношения (на $\mathbb{N}$ ) с одноэлементными областями истинности.

Структуры вида $\mathfrak{A}=\langle A ; \varepsilon\rangle$ с единственным бинарным отношением $\varepsilon$, понимаемым как отношение принадлежности, называются $\in$-структурами. Также будут рассматриваться структуры вида $\mathfrak{A}=\langle A ; \varepsilon, \ldots\rangle$, которые могут содержать, кроме $\varepsilon$, другие отношения, составляющие (возможно, пустой) списоK .... .

\footnotetext{
2Мы употребляем слово "(реляционная) структура" в том смысле, в каком обычно (скажем, в [39]) употребляется слово “модель", с той, однако, разницей, что универсумом структуры может быть не только множество, но и собственный класс - наиболее важные структуры ниже будут как раз иметь собственные классы в качестве универсумов.
} 
Более общо, если $\mathscr{L}$ - язык, содержащий реляционные символы $r_{1}, \ldots, r_{n}$, и арность каждого $r_{i}$ равна арности отношения $R_{i}$, то $\mathfrak{A}=\left\langle A ; R_{1}, \ldots, R_{n}\right\rangle$ называется $\mathscr{L}$-структурой. В этом случае для каждой $\mathscr{L}$-формулы $\varphi$ определяется ее релятивизация (или интерпретация) $\varphi^{\mathfrak{A}}$ посредством замены всякого вхождения любого реляционного символа $r_{i}, i=1, \ldots, n$, на $R_{i}$ и ограничения каждого квантора $\varphi$ к $A$. (Таким образом, $\exists x$ меняется на $\exists x \in A$, а $\forall x$ на $\forall x \in A$.) Обозначение $\varphi^{\mathfrak{A}}$ применимо также, когда некоторые или все свободные переменные $\varphi$ заменены элементами $A$ (которые в этом случае называются параметрами).

Если универсум $A$, а тогда, вообще говоря, и все отношения $R_{i}$ структуры $\mathfrak{A}=\left\langle A ; R_{1}, \ldots, R_{n}\right\rangle$ - собственные классы, определенные (в ZFC) некоторыми $\in$-формулами, то $\varphi^{\mathfrak{A}}$ также представляет собой $\in$-формулу, полученную из $\varphi$ соответственно определению, т.е. например $\exists x \ldots$ меняется на $\exists x(\alpha(x) \wedge \ldots)$, где $\alpha(x)$ есть $\in$-формула, определяющая $A$, а $R_{i}\left(x_{1}, \ldots, x_{m}\right)$ меняется на $\rho_{i}\left(x_{1}, \ldots, x_{m}\right)$, где $\rho_{i}$ есть $\in$-формула, определяющая $R_{i}$, a $m=m(i)$ обозначает арность $R_{i}$.

Понятно, что если $\varphi-$ замкнутая $\mathscr{L}$-формула с параметрами из $A$, то $\varphi^{\mathfrak{A}}$ выражает истинность $\varphi$ в $\mathfrak{A}$. Для структур, чьи универсумы являются множествами, имеется другое определение истинности, или выполнимости, записываемое в виде $\mathfrak{A} \models \varphi$ (см. [39; 1.3]), в котором сама формула $\varphi$ (понимаемая как строка символов определенного вида, т.е. как множество - после некоторых уточнений) становится переменной. $\mathrm{K}$ счастью, в этом случае $\varphi^{\mathfrak{A}}$ равносильно $\mathfrak{A}=\varphi$. (В этом утверждении есть не очевидные сразу детали, которых мы, однако, не будем касаться.)

Более широкий класс реляционных структур образуют инвариантные структуры, т.е. те, которые имеют вид $\mathfrak{A}=\left\langle A ; R_{1}, \ldots, R_{n} ; \mathrm{E}\right\rangle$, где $\mathrm{E}$ - отношение эквивалентности на $A$, а отношения $R_{i}$ являются Е-инвариантными независимо по каждому из аргументов. В этом случае равенство, в формулах соответствующего языка, интерпретируется в $\mathfrak{A}$ через отношение Е. Обычные реляционные структуры, как выше, соответствуют случаю, когда $\mathrm{E}$ - равенство.

Любая инвариантная структура $\mathfrak{A}=\left\langle A ; R_{1}, \ldots, R_{n} ; \mathrm{E}\right\rangle$ естественным образом превращается в обычную посредством факторизации по $\mathrm{E}$ с переходом к классам эквивалентности $[a]_{\mathrm{E}}, a \in A$, и с соответствующей факторизацией отношений $R_{i}$. Это, однако, плохо тем, что, для структур $\mathfrak{A}$, чьими универсумами $A$ являются собственные классы, классы эквивалентности $[a]_{\mathrm{E}}$ также могут быть собственными классами, что во многих отношениях неудобно. В ряде случаев (см., например, замечание 1.6) эту трудность можно обойти, выбрав в каждом классе эквивалентности $[a]_{\mathrm{E}}$ (собственном классе) один элемент, или хотя бы непустое множество элементов. В других важных случаях такая редукция к множествам невозможна. Но с точки зрения приложений к таким метаматематическим вопросам, как непротиворечивость или консервативность, инвариантные структуры применимы в равной мере с обычными.

1b. Элементарные расширения и прямые пределы. Теперь предположим, что $\mathfrak{A}=\left\langle A ; R_{1}, \ldots, R_{n}\right\rangle$ и $\mathfrak{A}^{\prime}=\left\langle A^{\prime} ; R_{1}^{\prime}, \ldots, R_{n}^{\prime}\right\rangle$ являются $\mathscr{L}$-структурами для одного и того же языка $\mathscr{L}$. Вложение $\pi: A \rightarrow A^{\prime}$ называется элементарным, когда мы имеем $\varphi^{\mathfrak{A}} \Leftrightarrow(\pi \varphi)^{\mathfrak{A}^{\prime}}$ для любой замкнутой $\mathscr{L}$-формулы 
с параметрами из $A$, где формула $\pi \varphi$ получается из $\varphi$ заменой каждого $x \in A$, встречающегося в $\varphi$ в роли параметра, на $\pi(x)$. Также говорят, что $\mathfrak{A}^{\prime}$ называется элементарным расширением $\mathfrak{A}$ (посредством $\pi$ ) в этом случае.

ОПРЕДЕЛЕНИЕ 1.1 (прямые пределы). Пусть $\lambda$ - предельный ординал. Цепъю элементарных структур или просто элементарной цепъю длины $\lambda$ называется последовательность структур $\mathfrak{A}_{\xi}=\left\langle A_{\xi} ; R_{1}^{\xi}, \ldots, R_{n}^{\xi}\right\rangle, \xi<\lambda(n$ не зависит от $\xi$ ), вместе с системой элементарных вложений $\pi_{\xi \eta}: A_{\xi} \rightarrow A_{\eta}$ $\left(\xi \leqslant \eta<\lambda\right.$, каждое $\pi_{\xi \xi}$ является тождественным отображением), удовлетворяющей условию $\pi_{\xi \zeta}=\pi_{\eta \zeta} \circ \pi_{\xi \eta}$.

Прямым пределом такой цепи является структура $\mathfrak{A}=\left\langle A_{\lambda} ; R_{1}^{\lambda}, \ldots, R_{n}^{\lambda}\right\rangle$, определяемая следующим образом. Положим

$$
A_{\xi}^{\prime}=A_{\xi} \backslash \bigcup_{\eta<\xi} \pi_{\eta \xi} " A_{\eta} \quad \text { и } \quad A_{\lambda}=\left\{\langle\xi, a\rangle: \xi<\lambda \wedge a \in A_{\xi}^{\prime}\right\} .
$$

Пусть $1 \leqslant k \leqslant n$. Все $R_{k}^{\xi}, \xi<\lambda$, имеют, очевидно, одну и ту же арность $s=s(k) \in \mathbb{N}$. Если $\left\langle\xi_{1}, a_{1}\right\rangle, \ldots,\left\langle\xi_{s}, a_{s}\right\rangle$ принадлежат $A$ и $\xi=\sup \left\{\xi_{1}, \ldots, \xi_{s}\right\}$, то мы определим $R_{k}^{\lambda}\left(\left\langle\xi_{1}, a_{1}\right\rangle, \ldots,\left\langle\xi_{s}, a_{s}\right\rangle\right)$, когда $R_{k}^{\xi}\left(\pi_{\xi_{1}, \xi}\left(a_{1}\right), \ldots, \pi_{\xi_{s}, \xi}\left(a_{s}\right)\right)$. Этим определение предельной структуры $\mathfrak{A}$ закончено. Дополнительно определяем, для каждого $\xi<\lambda$, вложение $\pi_{\xi \lambda}: A_{\xi} \rightarrow A_{\lambda}$ так. Пусть $a \in A_{\xi}$. Существует наименьший ординал $\eta \leqslant \xi$ такой, что $a=\pi_{\eta \xi}\left(a^{\prime}\right)$ для некоторого $a^{\prime} \in A_{\eta}$. (Например, $\eta=\xi$ при $a \in A_{\xi}^{\prime}$.) Понятно, что тогда $a^{\prime} \in A_{\eta}^{\prime}$. Положим $\pi_{\xi \lambda}(a)=$ $\left\langle\eta, a^{\prime}\right\rangle$.

Построение прямого предела может быть произведено и в случае элементарных цепей длины Ord (т.е. $\mathfrak{A}_{\xi}$ задано для каждого ординала $\xi$ ). В этом случае универсум $A_{\infty}=\left\{\langle\xi, a\rangle: \xi \in\right.$ Ord $\left.\wedge a \in A_{\xi}^{\prime}\right\}$ предельной структуры $\mathfrak{A}_{\infty}$ может быть собственным классом, даже если все $A_{\xi}$ - множества, но предельное вложение $e_{0 \infty}: A_{0} \rightarrow A_{\infty}$ все равно определяется аналогично случаю $\lambda \in$ Ord.

Мы опускаем доказательство следующей хорошо известной теоремы, в сущности довольно простое, хотя и достаточно трудоемкое, если все делать аккуратно. Доказательство см., например, в [39]: теорема 3.1.13 для случая, когда каждое $\pi_{\xi \eta}$ - тождественное отображение и, соответственно, $A=\bigcup_{\xi} A_{\xi}$, к которому общий случай легко сводится.

ТЕОрема 1.2. В ситуации, описанной в определении 1.1, структура $\mathfrak{A}_{\lambda}$ является элементарным расширением каждой структуры $\mathfrak{A}_{\xi}$ при отображении $\pi_{\xi \lambda}$. При $\xi<\eta \leqslant \lambda$ выполнено $\pi_{\xi \lambda}=\pi_{\eta \lambda} \circ \pi_{\xi \eta}$. То же для $\mathfrak{A}_{\infty}$ и иепей длины Ord.

1с. Насыщенные структуры и расширения. Наиболее важны для нестандартного анализа элементарные расширения, присоединяющие к исходной структуре такие элементы, существование которых в определенном смысле совместимо со свойствами данной структуры. Поскольку мы рассматриваем в основном $\in$-структуры, следующее центральное определение выглядит несколько проще, чем в общем случае (см., например, [39; гл. 5]).

ОПРЕДЕЛЕНИЕ 1.3. Пусть $\kappa$ - произвольный бесконечный кардинал. 
(i) Структура $\mathfrak{A}=\langle A ; \varepsilon, \ldots\rangle$ называется $\kappa$-насыщенной, если для любого множества $X \subseteq A$ мощности сard $X<\kappa$ из непустоты $\varepsilon$-пересечения каждого конечного $X^{\prime} \subseteq X$ в $\mathfrak{A}^{3}$ следует и непустота $\varepsilon$-пересечения самого $X$ в $\mathfrak{A}$.

(ii) Структура $\mathfrak{A}^{\prime}=\left\langle A^{\prime} ; \varepsilon^{\prime}, \ldots\right\rangle$ называется $\kappa$-насыщенной над структурой $\mathfrak{A}=\langle A ; \varepsilon, \ldots\rangle$ при вложении $\pi: A \rightarrow A^{\prime}$, если для любого $X \subseteq A$ мощности card $X<\kappa$ из непустоты $\varepsilon$-пересечения каждого конечного $X^{\prime} \subseteq X$ в $\mathfrak{A}$ следует непустота $\varepsilon^{\prime}$-пересечения множества $\pi X=\{\pi(x): x \in X\}$ в $\mathfrak{A}^{\prime}$.

Таким образом, $\kappa$-насыщенная структура - это та, которая $\kappa$-насыщена над самой собой. Мы увидим, что насыщенные структуры могут быть получены в виде пределов возрастающих цепей последовательных насыщений.

Пример 1.4. Рассмотрим $\in$-структуру $\mathfrak{P}=\langle P ; \in\rangle$, где $P=\mathscr{P}(\mathbb{N})$ (множество всех подмножеств натурального ряда $\mathbb{N}$ ). Каждое $n \in \mathbb{N}$ отождествляется с множеством $\{0,1, \ldots, n-1\}$, а потому $\mathbb{N} \subseteq P$.

Допустим, что $\in$-структура $\mathfrak{A}=\langle A ; \varepsilon\rangle \aleph_{1}$-насыщена над структурой $\mathfrak{P}$ при элементарном вложении $\pi: P \rightarrow A$. (Например, $\mathfrak{A}$ может быть $\aleph_{1}$-насыщенным элементарным расширением $\mathfrak{P}$.) В частности, $A$ содержит элемент $* \mathbb{N}=\pi(\mathbb{N})$ и элементы ${ }^{*} n=\pi(n), n \in{ }^{*} \mathbb{N}$, причем мы имеем ${ }^{*} k \varepsilon^{*} n \varepsilon{ }^{*} \mathbb{N}$ при $k \in n \in$ $\mathbb{N}$. Множество $X \subseteq P$ всех множеств вида $x_{n}=\{k \in \mathbb{N}: k \geqslant n\}, n \in \mathbb{N}$, счетно, и каждое конечное $X^{\prime} \subseteq X$ имеет, очевидно, непустое $\in$-пересечение (т.е. обычное пересечение), а потому по выбору $\mathfrak{A}$ найдется элемент $a \in A, \varepsilon$ принадлежащий всем множествам $\pi\left(x_{n}\right)$. Отсюда легко вывести $a \in{ }^{*} \mathbb{N}$ и $n \varepsilon a$ для всех $n \in \mathbb{N}$, т.е., в обычной интерпретации, $a$ - бесконечно большой элемент $* \mathbb{N}$ в $\mathfrak{A}$.

ТеОремА 1.5. Пусть $\kappa-$ бесконечный кардинал. Каждая структура вида $\mathfrak{A}=\left\langle A ; \varepsilon, R_{1}, \ldots, R_{n}\right\rangle$ ( $A$ - множество или класс) допускает элементарное вложение:

(i) в структуру, $\kappa^{+}$-насыщенную над $\mathfrak{A}$;

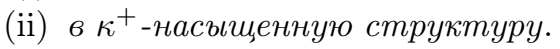

Если универсум $A=|\mathfrak{A}|$ - бесконечное множество мощности $\lambda$, то элементарные расширения в (i) $и$ (ii) могут быть выбраны с универсумами мощно$c m u \leqslant \lambda^{\kappa}$.

ДокАЗАТЕЛЬство. Понятно, что (ii) следует из (i) посредством построения цепи последовательных $\kappa$-насыщающих элементарных расширений, которая начинается с $\mathfrak{A}$, имеет длину $\kappa^{+}$(следующий за $\kappa$ кардинал) и использует (i) на непредельных шагах и теорему 1.2 на предельных шагах. ${ }^{4}$ (Надо также помнить, что $\lambda^{\kappa} \kappa^{+} \leqslant \lambda^{\kappa} 2^{\kappa}=(2 \lambda)^{\kappa}=\lambda^{\kappa}$.) Ниже следует доказательство (i).

Возьмем $I=[\kappa]^{<\omega}$ (множество всех конечных подмножеств $\kappa$ ) в качестве множества индексов для ультрапроизведения. Семейство всех множеств вида $I_{\alpha}=\{i \in I: \alpha \in i\}, \alpha<\kappa$, удовлетворяет условию конечных пересечений, т.е.

\footnotetext{
3Это означает существование $a \in A$, являющегося общим $\varepsilon$-элементом каждого $x \in X^{\prime}$.

${ }^{4}$ На самом деле, насыщенное расширение можно получить и в форме обычной однократной ультрастепени исходной структуры, но при помощи довольно сложного типа ультрафильтров, называемых хорошими ультрафильтрами. См. об этом [39; 6.1], особенно теорему 6.1.8.
} 
каждое конечное семейство множеств указанного вида имеет непустое пересечение. Поэтому найдется ультрафильтр $U \subseteq \mathscr{P}(I)$, содержащий все множества вида $I_{\alpha}, \alpha<\kappa$. Такие ультрафильтры названы в [25] адекватными.

Для построения ультрастепени $\mathfrak{A}^{I} / U={ }^{*} \mathfrak{A}=\left\langle{ }^{*} A ;{ }^{*} \varepsilon,{ }^{*} R_{1}, \ldots,{ }^{*} R_{n}\right\rangle$ структуры $\mathfrak{A}$ по ультрафильтру $U$ рассматривается совокупность $A^{I}$ всех функций $f: I \rightarrow A$. Положим

$$
f={ }_{U} g, \quad \text { когда } \quad\{i: f(i)=g(i)\} \in U,
$$

для любых $f, g \in A^{I}$. Ясно, что $={ }_{U}$ является отношением эквивалентности на $A^{I}$, а потому можно рассмотреть классы эквивалентности $[f]_{U}=\left\{g \in A^{I}\right.$ : $\left.f={ }_{U} g\right\}$. Наконец, положим ${ }^{*} A=\left\{[f]_{U}: f \in A^{I}\right\}$. Понятно, что card ${ }^{*} A \leqslant \lambda^{\kappa}$.

ЗАмЕчание 1.6. Если $A$ - собственный класс, то, согласно данному определению, каждый класс эквивалентности $[f]_{U}$ может быть собственным классом, а $* \mathfrak{A}-$ классом классов. По формальным соображениям, совокупности такого рода трудно рассматривать в ZFC. Однако имеется удовлетворительный выход: урезать каждый собственный класс $[f]_{U}$ до множества всех функций $g \in[f]_{U}$, имеющих наименьший ранг по фон Нейману $\operatorname{rk} g$ среди всех $g \in[f]_{U}$. С таким изменением все еще $f={ }_{U} g \Leftrightarrow[f]_{U}=[g]_{U}$, однако сама $f$ не обязательно принадлежит $[f]_{U}$.

Далее, мы определяем $[f]_{U}{ }^{*} \varepsilon[g]_{U}$, когда $\{i: f(i) \varepsilon g(i)\} \in U$, и вообще

$$
{ }^{*} R_{k}\left(\left[f_{1}\right]_{U}, \ldots,\left[f_{s}\right]_{U}\right), \quad \text { когда } \quad\left\{i: R_{k}\left(f_{1}(i), \ldots, f_{s}(i)\right)\right\} \in U \quad(1 \leqslant k \leqslant n),
$$

где $s$ есть арность $R_{k}$ и $f_{1}, \ldots, f_{s} \in A^{I}$. Корректность определений ${ }^{*} \varepsilon$ и ${ }^{*} R_{k}$, т.е. независимость правых частей от выбора представителей в классах эквивалентности, легко следует из того факта, что $U$ - ультрафильтр. Наконец, для определения элементарного вложения $\mathfrak{A}$ в $* \mathfrak{A}$, положим ${ }^{*} x=\left[\mathbf{f}_{x}\right]_{U}$ для всякого $x \in A$, где $\mathbf{f}_{x} \in A^{I}$ - функция-константа, определенная условием $\mathbf{f}_{x}(i)=x$ для Bcex $i \in I$.

Доказательство следующего хорошо известного результата (см. [39; 4.1.9]) мы опускаем. Для удобства обозначим через $\mathscr{L}$ язык, содержащий реляционные символы соответствующей арности для $\varepsilon$ и всех $R_{i}$. Для каждой $\mathscr{L}$ формулы $\varphi$ ее релятивизации $\varphi^{\mathfrak{A}}$ и $\varphi^{*} \mathfrak{A}$ определяются как указано в п. 1а.

ПреДЛОЖЕНИЕ 1.7 (теорема Лося). $B$ наших обозначениях для любой $\mathscr{L}$-формулы $\varphi\left(x_{1}, \ldots, x_{n}\right)$ и любого набора $f_{1}, \ldots, f_{n} \in A^{I}$ выполнено

$$
\varphi^{*} \mathfrak{A}\left(\left[f_{1}\right]_{U}, \ldots,\left[f_{n}\right]_{U}\right) \Leftrightarrow\left\{i \in I: \varphi^{\mathfrak{A}}\left(f_{1}(i), \ldots, f_{n}(i)\right)\right\} \in U .
$$

СлеДСтвиЕ 1.8. Отображение $x \mapsto{ }^{*} x-$ элементарное вложение $\mathfrak{A}$ в * $\mathfrak{A}$.

Для доказательства теоремы остается проверить, что структура $* \mathfrak{A} \quad \kappa^{+}$-насыщена над $\mathfrak{A}$. Рассмотрим произвольное множество $X=\left\{x_{\alpha}: \alpha<\kappa\right\} \subseteq A$ мощности $<\kappa^{+}$, т.е. $\leqslant \kappa$. Предположим, что для каждого конечного $Y \subseteq X$ найдется элемент $z_{Y} \in A$, удовлетворяющий $z_{Y} \varepsilon y$ для всех $y \in Y$. Если $i=\left\{\alpha_{1}, \ldots, \alpha_{m}\right\} \in I$, то положим $Y(i)=\left\{x_{\alpha_{1}}, \ldots, x_{\alpha_{m}}\right\}$ и $f(i)=z_{Y(i)}$. Покажем, что $[f]^{*} \varepsilon^{*} x$ выполнено для любого $x \in X$. Согласно предложению 1.7 , достаточно показать, что множество $u_{\alpha}=\left\{i \in I: f(i) \in x_{\alpha}\right\}$ принадлежит $U$ 
при любом $\alpha<\kappa$. Здесь мы используем специфику выбора ультрафильтра $U$. Именно, из построения легко следует, что $I_{\alpha}=\{i: \alpha \in i\} \subseteq u_{\alpha}$. Но $I_{\alpha} \in U$ по выбору $U$, стало быть, и $u_{\alpha}$ принадлежит $U$. Теорема 1.5 доказана.

1d. “Эффективные” насыщенные расширения. Нетрудно проверить, что любая бесконечная $\kappa$-насыщенная $\in$-структура, удовлетворяющая подходящему минимальному набору требований (например, аксиомам ZFC без Подстановки), имеет мощность строго больше $\kappa$, а потому, в указанных условиях, абсолютно насыщенная, т.е. $\kappa$-насыщенная для каждого кардинала $\kappa$, структура должна иметь своим универсумом собственный класс. На первый взгляд, построение абсолютно насыщенных расширений при помощи результатов пп. 1b, 1с не составляет труда: строим элементарную цепь $\left\{\mathfrak{A}_{\xi}\right\}_{\xi \in \mathrm{Ord}}$, используя прямой предел на предельных шагах (теорема 1.2) и насыщающие расширения (теорема 1.5) с неограниченно возрастающим кардинальным параметром на непредельных шагах. Однако при ближайшем рассмотрении задача становится более сложной.

В самом деле, теорема 1.5 неэффективна в том смысле, что данное ее доказательством расширение зависит от выбора ультрафильтра, так что для построения приходится использовать аксиому глобального выбора в случае, когда выбирающая “функция" имеет областью определения класс всех ординалов, для выбора подходящего ультрафильтра на каждом шагу $\xi \in$ Ord. Однако имеется и альтернативный метод, лишенный этого недостатка. Его суть состоит в том, чтобы сделать построение насыщающего расширения "эффективным", т.е. не зависящим от произвольного выбора ультрафильтра или чего-либо еще. При этом удается обойтись без аксиомы глобального выбора и, таким образом, остаться в ZFC.

Построєние 1.9 (“эффективное" насыщение). Зафиксируем бесконечный кардинал $\kappa$ и структуру $\mathfrak{A}=\langle A ; \varepsilon, \ldots\rangle$, универсум $A$ которой является множеством или собственным классом. Следующее построение итерированного ультрапроизведения дано в [39; 6.5] (в несколько иных обозначениях), и все относящиеся к нему приведенные ниже факты взяты оттуда.

1) Упорядочим множество $\mathscr{P}(\kappa)$ (всех множеств $X \subseteq \kappa$ ) так, что $X<Y$, когда наименьший элемент симметрической разности $X \Delta Y=(X \backslash Y) \cup(Y \backslash X)$ принадлежит $Y$. Определим кардинал $\delta=2^{\kappa}$ и упорядочим множество $\mathscr{P}(\kappa)^{\delta}$ (всех $\delta$-последовательностей множеств $X \subseteq \kappa$ ) лексикографически: $s \prec t$, когда $s(\xi)<t(\xi)$ (в смысле предшествующего определения), где $\xi=\min \{\xi<\delta$ : $s(\xi) \neq t(\xi)\}$.

2) Рассмотрим множество $\mathscr{S}$ всех последовательностей $s=\left\{X_{\xi}\right\}_{\xi<\delta} \in \mathscr{P}(\kappa)^{\delta}$ таких, что множество $U_{s}=\operatorname{ran} s=\left\{X_{\xi}: \xi<\delta\right\}$ является ультрафильтром $($ над $\kappa)$. Множество $\mathscr{S}\left(\right.$ как и $\left.\mathscr{P}(\kappa)^{\delta}\right)$ линейно упорядочено порядком $\prec$. Итак, $\left\{U_{s}\right\}_{s \in \mathscr{S}}$ представляет собой линейное (не полное) упорядочение, с повторениями, множества всех ультрафильтров над $\kappa$. Мы используем его, чтобы построить такое элементарное расширение $\mathfrak{A}$, которое включает в себя все ультрастепени вида $\mathfrak{A}^{\kappa} / U$, где $U-$ ультрафильтр над $\kappa$. K чему это приведет см. ниже. 
3) Пусть $S=\left\{s_{1}<s_{2}<\cdots<s_{n}\right\} \subseteq \mathscr{S}-$ конечное множество. Через $U_{S}$ обозначим совокупность всех множеств $X \subseteq \kappa^{S}$, удовлетворяющих

$$
U_{s_{n}} \xi_{n} \ldots U_{s_{2}} \xi_{2} U_{s_{1}} \xi_{1} \quad\left(\left\langle\xi_{1}, \xi_{2}, \ldots, \xi_{n}\right\rangle \in X\right),
$$

где $U_{s} \xi \Phi(\xi)$ означает, что множество $\{\xi: \varphi(\xi)\}$ принадлежит $U_{s}$. Известно, что $U_{S}$ - ультрафильтр над $\kappa^{S}$, причем ультрастепень по $U_{S}$ тождественна последовательному расширению ультрастепенями по ультрафильтрам $U_{s_{1}}, U_{s_{2}}, \ldots, U_{s_{n}}$. Рассмотрим $U_{S}^{\prime}$ всех множеств $X^{\prime} \subseteq \kappa^{\mathscr{S}}$ вида $X^{\prime}=X \uparrow \mathscr{S}=\left\{i \in \kappa^{\mathscr{S}}\right.$ : $i \uparrow S \in X\}$, где $X \in U_{S}$. Тогда объединение $U=\bigcup_{S \subseteq \mathscr{S} \text { конечно }} U_{S}^{\prime}$ является ультрафильтром в алгебре В всех множеств $X^{\prime} \subseteq \kappa^{\mathscr{S}}$ вида $X^{\prime}=X \uparrow \mathscr{S}$, где $X \subseteq \kappa^{S}$ произвольно, а множество $S \subseteq \mathscr{S}$ конечно. Следуя терминологии [39], множества из алгебры В можно назвать конечно определенными в полной степени $\mathscr{P}\left(\kappa^{\mathscr{S}}\right)$.

4) Продолжая построение, положим $F=\bigcup_{S \subseteq \mathscr{S} \text { конечно }} F_{S}$, где $F_{S}$ есть совокупность всех функций $f: \kappa^{\mathscr{S}} \rightarrow A$ таких, что $i \uparrow S=j \uparrow S \Rightarrow f(i)=f(j)$. ( $F$ является множеством или собственным классом вместе с универсумом $A$ данной структуры $\mathfrak{A}$.) Опять следуя терминологии [39], функции из $F$ будем называть конечно определенными. Само итерированное ультрапроизведение ${ }^{*} \mathfrak{A}=F / U=\left\langle{ }^{*} A ;{ }^{*} \varepsilon, \ldots\right\rangle$ имеет своим универсумом ${ }^{*} A=\left\{[f]_{U}: f \in F\right\}$, где $[f]_{U}=\left\{g \in F: f={ }_{D} g\right\}$ (с поправкой, указанной в замечании 1.6), а $f={ }_{U} g$ означает, что множество $I_{f g}=\left\{i \in \kappa^{\mathscr{S}}: f(i)=g(i)\right\}$ принадлежит $U$. (Заметим, что $I_{f g}$ принадлежит В для любых $f, g \in F$.) Остается определить отношение $[f]_{U}{ }^{*} \varepsilon[g]_{U}$, когда $\left\{i \in \kappa^{\mathscr{S}}: f(i) \in g(i)\right\} \in U$ (см. доказательство теоремы 1.5$)$, и аналогично определить $*$-продолжения ${ }^{*} R$ всех отношений $R$, входящих в список ... отношений данной структуры $\mathfrak{A}$.

5) Положим ${ }^{*} x=\left[\mathbf{f}_{x}\right]_{U}$ для всякого $x \in A$, где $\mathbf{f}_{x} \in F$ - функция-константа, $\mathbf{f}_{x}(i)=x$ для всех $i \in \kappa^{\mathscr{S}}$. Хотя совокупность (множество или класс вместе с $A) F$ и не содержит всех функций из $\kappa^{\mathscr{S}}$ в $A$, но $F$ и алгебра В согласованы между собой так, что теорема Лося 1.7 и следствие 1.8 сохраняют силу (см., например, 6.5.5 и 6.5.6 в [39]), так что $x \mapsto{ }^{*} x$ - элементарное вложение $\mathfrak{A}$ в * $\mathfrak{A}$.

6) Для доказательства того, что структура * $\mathfrak{A} \kappa^{+}$-насыщена над $\mathfrak{A}$ при указанном отображении, рассмотрим произвольное множество $X \subseteq A$ мощности $\leqslant \kappa$, удовлетворяющее условию непустоты $\varepsilon$-пересечения любого конечного $X^{\prime} \subseteq X$. Мы видели (см. доказательство теоремы 1.5), что существует ультрафильтр над множеством $I=[\kappa]^{<\omega}$ такой, что соответствующая ультрастепень структуры $\mathfrak{A} \kappa^{+}$-насыщена над $\mathfrak{A}$. Ультрафильтр с таким свойством существует и над $\kappa$, поскольку $\operatorname{card} I=\kappa,-$ а тогда он имеет вид $U_{s}$ для подходящего $s \in \mathscr{S}$. Итак, ультрастепень $\mathfrak{A}^{\kappa} / U_{s} \quad \kappa^{+}$-насыщена над $\mathfrak{A}$ при естественном вложении. Иными словами, имеется функция $h: \kappa \rightarrow A$, удовлетворяющая $[h]_{U_{s}}{ }^{*} \varepsilon^{*} x$ для всех $x \in X$, т.е., по теореме Лося, $E_{x}=\{\xi<\kappa: h(\xi) \in x\} \in U_{s}$ для всех $x \in X$.

Определим функцию $f: \kappa^{\mathscr{S}} \rightarrow A$ равенством $f(i)=h(i(s))$ для всех $i \in \kappa^{\mathscr{S}}$. Понятно, что $f \in F$, фактически $f \in F_{\{s\}}$. При любом $x \in A$ множество $E_{x}^{\prime}=\left\{i \in \kappa^{\mathscr{S}}: f(i) \in x\right\}$ совпадает с $E_{x} \uparrow \mathscr{S}=\left\{i \in \kappa^{\mathscr{S}}: i(s) \in E_{x}\right\}$, а потому, по определению $U$, мы имеем $E_{x}^{\prime} \in U \Leftrightarrow E_{x} \in U_{s}$. Значит, по предыдущему, $E_{x}^{\prime} \in U$ для любого $x \in X$. Отсюда, по теореме Лося в обратном направлении 
для $U$ (см. ссылку выше), мы имеем $[f]_{U}{ }^{*} \varepsilon^{*} x$ в * $\mathfrak{A}$ для любого $x \in X$, что и требовалось.

СлЕДСТвиЕ 1.10. В обозначениях построения 1.9 структура ${ }^{*} \mathfrak{A}=\left\langle{ }^{*} A\right.$; $\left.{ }^{*} \varepsilon, \ldots\right\rangle$ есть "эффективное", однозначно и недвусмысленно определенное элементарное расширение структуры $\mathfrak{A}=\langle A ; \varepsilon, \ldots\rangle, \kappa^{+}$-насыщенное над $\mathfrak{A}$, при отображении $x \mapsto{ }^{*} x$.

ЗАмЕчАниЕ 1.11 (ограниченные вложения). Вложение $x \mapsto{ }^{*} x$ структуры $\mathfrak{A}=\langle A ; \varepsilon, \ldots\rangle$ в структуру ${ }^{*} \mathfrak{A}=\left\langle{ }^{*} A ;{ }^{*} \varepsilon, \ldots\right\rangle$ называется ограниченным, если для любого $y \in{ }^{*} A$ существует $a \in A$, удовлетворяющее $y^{*} \varepsilon^{*} a$. Это очень важное свойство расширений $\in$-структур. Расширения типа 1.9-1.10 не обязательно ограничены, однако имеется удобное достаточное условие. Структура вида $\mathfrak{A}=\langle A ; \varepsilon, \ldots\rangle$ называется почти универсальной [34], если ко всякому множеству $X \subseteq A$ найдется элемент $a \in A$, удовлетворяющий $x \varepsilon a$ для всех $x \in X$. В этом случае $A$ - собственный класс (или, иначе, $a \varepsilon a$ для некоторого $a$ ).

Мы утверждаем, что, в условиях 1.9 , если $\mathfrak{A}$ - почти универсальная структура, то $x \mapsto{ }^{*} x$ - ограниченное вложение $\mathfrak{A}$ в ${ }^{*} \mathfrak{A} u^{*} \mathfrak{A}-$ также почти универсальная структура. Предположим, что (в обозначениях 1.9$) y=[f]_{U}$, где $f: \kappa^{\mathscr{S}} \rightarrow A$ - конечно определенная (в смысле пункта 4) выше) функция. Положим $a=\operatorname{ran} f$. Тогда $f(i) \in \mathbf{f}_{a}(i)=a$ для всех $i \in \kappa^{\mathscr{S}}$, откуда следует $[f]_{U}=y^{*} \varepsilon^{*} a=\left[\mathbf{f}_{a}\right]_{U}$, что и требовалось. Теперь мы сразу имеем и почти универсальность $* \mathfrak{A}$.

Для построения ограниченных элементарных расширений тех $\in$-структур, которые не являются почти универсальными, например, структур вида $\langle X ; \in \mid X\rangle$, где $X$ - любое множество, достаточно изменить конструкцию ультрастепени в 1.9, оставив лишь такие (конечно определенные) функции $f: \kappa^{\mathscr{S}} \rightarrow A$, что для некоторого $a \in A$ выполнено $\forall x \in \operatorname{ran} f(x \varepsilon a)$. См. детали в [25; гл. 4].

1е. Абсолютно насыщенные расширения. Мы рассматривали структуры, насыщенность которых была ограничена некоторым кардиналом. Следующее определение вводит “абсолютную” версию этого понятия. Как указано выше, универсум абсолютно насыщенной структуры либо конечен, либо является собственным классом, а не множеством.

ОПРЕДЕЛЕНИЕ 1.12. Абсолютно насыщенной называется любая $\in$-структура, являющаяся $\kappa$-насыщенной для каждого кардинала $\kappa$.

ТЕОрема 1.13. Каждая почти универсальная в смысле замечания 1.11 структура $\mathfrak{A}=\langle A ; \varepsilon, \ldots\rangle \quad(A-$ множество или класс $)$ допускает элементарное вложсене в абсолютно насъщенную структуру ${ }^{*} \mathfrak{A}=\left\langle{ }^{*} A ;{ }^{*} \varepsilon, \ldots\right\rangle$, ограниченное в смысле замечания 1.11.

Такое расширение * $\mathfrak{A}$ может быть получено из $\mathfrak{A}$ “эффективным", однозначным и вполне недвусмысленным построением.

ДоказАтельство. Случай 1: $A$ (а тогда и $\mathfrak{A})$ - множество. Здесь все просто: строим по трансфинитной индукции $\in$-структуры $\mathfrak{A}_{\xi}=\left\langle A_{\xi} ; \varepsilon_{\xi}, \ldots\right\rangle, \xi \in$ Ord (все $A_{\xi}$ - множества) и элементарные вложения $e_{\eta \xi}: \mathfrak{A}_{\eta} \rightarrow \mathfrak{A}_{\xi}, \eta<\xi \in$ Ord, 
так, что $\mathfrak{A}_{0}=\mathfrak{A}$, каждая $\mathfrak{A}_{\xi+1}$ является элементарным и $(\operatorname{card}(\omega+\xi))^{+}$-насыщенным ${ }^{5}$ над $\mathfrak{A}_{\xi}$ расширением $\mathfrak{A}_{\xi}$, даваемым следствием 1.10 , а $e_{\xi, \xi+1}$ совпадает с соответствующим элементарным вложением $\mathfrak{A}_{\xi}$ в $\mathfrak{A}_{\xi+1}, e_{\eta, \xi+1}=e_{\xi, \xi+1}$ о $e_{\eta \xi}$ для всех $\eta<\xi$, наконец, каждое $e_{\xi, \xi+1}$ является ограниченным вложением в смысле следствия 1.10 и, кроме того, $\mathfrak{A}_{\lambda}-$ прямой предел последовательности $\left\{\mathfrak{A}_{\xi}\right\}_{\xi<\lambda}$ для каждого предельного ординала $\lambda$.

Возьмем в качестве ${ }^{*} \mathfrak{A}=\left\langle{ }^{*} A ;{ }^{*} \varepsilon, \ldots\right\rangle$ прямой предел $\mathfrak{A}_{\infty}$ последовательности $\left\{\mathfrak{A}_{\xi}\right\}_{\xi \in \text { Ord }}$ при указанной системе элементарных вложений, как в определении 1.1 , с соответствующим элементарным вложением $x \mapsto{ }^{*} x: A_{0} \rightarrow A_{\infty}={ }^{*} A$, т.е. $e_{0 \infty}$ в определении 1.1. Чтобы увидеть, что структура $* \mathfrak{A} \kappa$-насыщенна для любого кардинала $\kappa$, достаточно заметить, что каждая структура $\mathfrak{A}_{\xi+1}$ по построению является $\kappa$-насыщенной над $\mathfrak{A}_{\xi}$ при отображении $e_{\xi, \xi+1}$.

Наконец, мы утверждаем, что каждое $e_{0 \xi}$ есть ограниченное (элементарное) вложение $\mathfrak{A}_{0}$ в $\mathfrak{A}_{\xi}$, а $\mathfrak{A}_{\xi}$ - почти универсальная структура. При $\xi=\infty$ (т.е. предельный шаг после всех ординалов) имеем искомую ограниченность вложения $x \mapsto^{*} x$. Предельный шаг (включая и заключительный шаг $\infty$ ) здесь достаточно элементарен, а шаг $\xi \rightarrow \xi+1$ обеспечивается результатом замечания 1.11 (выделено там курсивом), согласно которому $e_{\xi, \xi+1}$ обеспечивает ограниченное вложение $\mathfrak{A}_{\xi}$ в $\mathfrak{A}_{\xi+1}$.

Случай 2: $A$ (а тогда и $\mathfrak{A})$ - собственный класс. Предыдущее рассуждение прямо не проходит, поскольку ни ZFC (с неформальным пониманием классов), ни даже теория классов GB не поддерживают индуктивное трансфинитное построение собственных классов. Однако задача может быть решена сведе́нием к случаю 1.

Положим $A^{\alpha}=A \cap \mathbf{V}_{\alpha}$ (где $\mathbf{V}_{\alpha}$ есть $\alpha$-й уровень иерархии фон Неймана) и $\varepsilon^{\alpha}=\varepsilon \uparrow A^{\alpha}$. Каждая структура $\mathfrak{A}^{\alpha}=\left\langle A^{\alpha} ; \varepsilon^{\alpha}, \ldots\right\rangle$ имеет множсество $A^{\alpha}$ своим универсумом, так что можно определить последовательность ее элементарных расширений $\mathfrak{A}_{\xi}^{\alpha}=\left\langle A_{\xi}^{\alpha} ; \varepsilon_{\xi}^{\alpha}, \ldots\right\rangle, \xi \in$ Ord, так, как указано в случае 1.

Здесь будет технически удобно принять поправку типа замечания 1.6 на всех шагах построения, даже при том, что у нас области $A_{\xi}^{\alpha}-$ множества, а не собственные классы. Положим $\kappa_{\xi}=\operatorname{card}(\omega+\xi)$ (см. случай 1 ) для каждого $\xi \in$ Ord. Соответствующие этому выбору $\kappa=\kappa_{\xi}$ множества $\mathscr{S}$ и $U$ (см. 1.9) условимся обозначать через $\mathscr{S}_{\xi}$ и $U_{\xi}$, в частности, $U_{\xi}$ - ультрафильтр в алгебре $\mathbf{B}_{\xi}$ всех конечно определенных множеств $X \subseteq I_{\xi}=\kappa_{\xi} \mathscr{S}_{\xi}$. Наконец, через $F_{\xi}^{\alpha}$ будем обозначать множество всех конечно определенных функций $f: I_{\xi} \rightarrow A_{\xi}^{\alpha}$. В этой символике построение 1.9 (пункт 4)) определяет $A_{\xi+1}^{\alpha}=\left\{[f]_{U_{\xi}}^{\alpha}: f \in F_{\xi}^{\alpha}\right\}$, где $[f]_{U_{\xi}}^{\alpha}=\left\{g \in F_{\xi}^{\alpha}: f={ }_{U_{\xi}} g\right\}$.

Модифицируя это определение, положим $A_{\xi+1}^{\alpha}=\left\{[f]_{U_{\xi}}^{\mathrm{abs}}: f \in F_{\xi}^{\alpha}\right\}$, где $[f]_{U_{\xi}}^{\mathrm{abs}}$ есть множество всех конечно определенных функций $g$ с областью определения $\operatorname{dom} g=I_{\xi}$ и любыми значениями, удовлетворяющих $f={ }_{U_{\xi}} g$ и имеющих наименьший ранг rkg среди всех таких функций $g$. (Подчеркнем, что для $g \in$ $[f]_{U_{\xi}}^{\mathrm{abs}}$ не обязательно выполнено $\operatorname{ran} g \subseteq A_{\xi}^{\alpha}$ и вообще $[f]_{U_{\xi}}^{\mathrm{abs}}$ зависит только от $f$ и ультрафильтра $U_{\xi}$.) Этим определяется индуктивный переход $\mathfrak{A}_{\xi}^{\alpha} \rightarrow \mathfrak{A}_{\xi+1}^{\alpha}$. Предельный шаг остается без изменений (прямой предел). Наконец, через $e_{\xi \eta}^{\alpha}$,

\footnotetext{
${ }^{5} \omega$ в выражении $\omega+\xi$ добавлено, чтобы сделать кардинал бесконечным при конечном $\xi$.
} 
$\alpha \in \operatorname{Ord}, \xi<\eta \in$ Ord, обозначается система элементарных вложений $\mathfrak{A}_{\xi}^{\alpha} \rightarrow \mathfrak{A}_{\eta}^{\alpha}$, возникающая при этом трансфинитном индуктивном построении.

$\mathrm{C}$ таким уточнением мы имеем для всех $\xi<\eta \in$ Ord и $\alpha<\beta \in$ Ord:

(*) $A_{\xi}^{\alpha} \subseteq A_{\xi}^{\beta}, \mathfrak{A}_{\xi}^{\alpha}=\mathfrak{A}_{\xi}^{\beta} \uparrow A_{\xi}^{\alpha}$ (т.е. $\varepsilon_{\xi}^{\alpha}=\varepsilon_{\xi}^{\beta} \uparrow A_{\xi}^{\alpha}$ и то же для всех отношений из списка ...) и наконец $e_{\xi \eta}^{\alpha}=e_{\xi \eta}^{\beta} \uparrow A_{\xi}^{\alpha}$.

(Простое доказательство по индукции опускается.) Теперь положим $\mathfrak{A}_{\xi}=$ $\bigcup_{\alpha \in \text { Ord }} \mathfrak{A}_{\xi}^{\alpha}$, т.е. $\mathfrak{A}_{\xi}=\left\langle A_{\xi} ; \varepsilon_{\xi}, \ldots\right\rangle$, где $A_{\xi}=\bigcup_{\alpha \in \text { Ord }} A_{\xi}^{\alpha}, \varepsilon_{\xi}=\bigcup_{\alpha \in \text { Ord }} \varepsilon_{\xi}^{\alpha}$, и то же для всех отношение из списка .... Из $(*)$ легко следует, что каждая структура $\mathfrak{A}_{\xi+1}$ является ультрастепенью $\mathfrak{A}_{\xi}$ типа 1.9 по ультрафильтру $U_{\xi}$, а на предельных шагах сохраняется прямой предел. Этим обходится трудность, связанная с индуктивным построением классов (см. начало случая 2), а дальше все аналогично случаю 1.

“Эффективность" построения * $\mathfrak{A}$ в обоих случаях следует из “эффективности" главного элемента конструкции - построения "эффективного" насыщения в 1.9. Теорема 1.13 доказана.

1f. Теория ограниченных множеств. Взяв универсум $\langle\mathbf{V} ; \in\rangle$ в роли структуры $\mathfrak{A}$ в теореме 1.13, мы получаем абсолютно насыщенное элементарное расширение всего универсума множеств V. Исследование таких расширений удобно проводить аксиоматическим методом, т.е. сначала доказать несколько общих, определяющих свойств - аксиом, а затем выводить остальные свойства из этих аксиом, уже не обращаясь к деталям построения.

В силу элементарности расширения, такая аксиоматизация абсолютно насыщенных элементарных расширений $\mathbf{V}$ должна включать ZFC. Новый аспект сообщает стандартное ядро расширения, т.е. образ универсума $\mathbf{V}$ при рассматриваемом вложении. Для адекватного отображения структуры такого расширенного универсума к обычному $\in$-языку добавляется еще один атомарный (неопределяемый) предикат стандартности st. B этом расширенном st- $\in$-языке можно определить разные нестандартные теории множеств, из которых для описания абсолютно насыщенных элементарных расширений универсума ZFC пригодна теория ограниченных множеств BST. Теория BST содержит следующие аксиомы:

$\mathbf{Z F C}^{\text {st }}$ : все формулы вида $\Phi^{\mathrm{st}}$, где $\Phi$ - аксиома $\mathbf{Z F C}$ в $\in$-языке.

Ограниченность: $\forall x \exists^{\text {st }} y \quad(x \in y)$.

Перенос: все формулы вида $\Phi^{\text {st }} \Leftrightarrow \Phi$, где $\Phi$ - замкнутая $\in$-формула со стандартными параметрами.

Базовая Идеализация: $\forall^{\text {st }} A_{0} \forall^{\text {st }} X \forall \psi$ (если $\psi$ - функция из $A_{0}$ в $\mathscr{P}(X)$, то $\left.\left[\forall^{\text {stfin }} A \subseteq A_{0} \exists x \in X \forall a \in A(x \in \psi(a)) \Leftrightarrow \exists x \in X \forall^{\text {st }} a \in A_{0}(x \in \psi(a))\right]\right)$.

Стандартизация: все формулы вида $\forall^{\mathrm{st}} X \exists^{\mathrm{st}} Y \forall^{\mathrm{st}} x(x \in Y \Leftrightarrow x \in X \wedge \Phi(x))$, где $\Phi(x)$ - любая st- $\in$-формула с любыми параметрами.

К обозначениям. Кванторы $\exists^{\text {st }} x \ldots, \forall^{\text {st }} x \ldots$ суть сокращения соответственно для $\exists x($ st $x \wedge \ldots)$ и $\forall x($ st $x \Rightarrow \ldots)$. Через $\Phi^{\text {st }}$ обозначается релятивизация $\in$-формулы $\Phi$ к структуре $\langle\mathbb{S} ; \in\rangle$, где $\mathbb{S}=\{x:$ st $x\}-$ класс всех стандартных множеств - т.е. все кванторы $\exists x, \forall x$ меняются на $\exists^{\text {st }} x, \forall^{\text {st }} x$. $\forall^{\text {stfin }} A$ означает: для каждого стандартного конечного $A$; конечность понимается в обычном смысле, принятом в ZFC. Здесь следует отметить, что ZF $\mathbf{Z C}^{\text {st }}$ и 
Перенос влекут $\mathbf{Z F C}$ в $\in$-языке ${ }^{6}$, а потому все обычные понятия на $\mathbf{Z F C - о с н о в е ~}$ сохраняют свой смысл и свойства. Подчеркнем, что схемы Выделения и Подстановки из ZFC выводятся в BST только в $\in$-языке и не распространяются, вообще говоря, на st- $\in$-формулы.

Мы будем использовать II для обозначения универсума всех множеств BST. Перенос означает, что $\mathfrak{S}=\langle\mathbb{S} ; \in\rangle$ является элементарной подструктурой универсума $\langle\mathbb{I} ; \in\rangle$. Ограниченность говорит, что класс $\mathbb{S} \in$-конфинален в универсуме. Базовая Идеализация - это, очевидно, аксиома типа насыщенности: в самом деле, вторую строку можно переписать в виде

$$
\left.\forall^{\text {stfin }} A \subseteq A_{0}\left(\bigcap_{a \in A} \psi(a) \neq \varnothing\right) \Leftrightarrow \bigcap_{a \in A_{0} \cap \mathbb{S}} \psi(a) \neq \varnothing\right) .
$$

При этом в BST даже без Базовой Идеализации можно доказать, что каждое стандартное конечное множество содержит только стандартные элементы, см. лемму 1.14(iv). Наконец, Стандартизация означает определенную полноту класса $\mathbb{S}$ всех стандартных множеств.

Чтобы показать, как работают аксиомы BST, приведем доказательства нескольких простых утверждений, собранных в следующую лемму. Некоторые из них будут использоваться ниже, в том числе без явных ссылок.

ЛЕмМА 1.14 (BST).

(i) Совокупность $\mathbb{N} \cap \mathbb{S}=\{n \in \mathbb{N}:$ st $n\}$ всех стандартных натуральных чисел - собственный начальный сегмент множества $\mathbb{N}$, не являющийся множеством.

(ii) Принцип индукции выполнен в $\in$-языке в области $\mathbb{N}$, а в st- $\in$-языке только в области $\mathbb{N} \cap \mathbb{S}$.

(iii) Всякая стандартно-конечная (т.е. ее длина $n$ принадлежит $\mathbb{N} \cap \mathbb{S}$ ) последовательность $s=\left\langle x_{1}, \ldots, x_{n}\right\rangle$ стандартных множеств $x_{n}$ сама стандартна.

(iv) Если $x$ - стандартное конечное множество, то $x \subseteq \mathbb{S}$.

(v) Eсли $x \in \mathbb{S}$ бесконечно, то $x \nsubseteq \mathbb{S}$.

(vi) Если $x \notin \mathbb{S}$, то объединение $\widehat{x}=\bigcup_{y \in \mathbb{S}, y \subseteq x} y$ всех стандартных подмножеств $x$ есть собственное подмножество $x$.

ДоказАтельство. (i) Пусть, напротив, $Y=\mathbb{N} \cap \mathbb{S}$ является множеством. Определим $\psi(n)=\mathbb{N} \backslash\{0,1, \ldots, n\}$ для каждого $n \in \mathbb{N}$. Левая часть эквивалентности [...] в Базовой Идеализации верна при $A_{0}=X=\mathbb{N}$ (каждое конечное множество в $\mathbb{N}$ ограничено сверху). Значит, верна и правая часть, т.е. существует $x \in \mathbb{N}$ такое, что $n<x$ для всех стандартных $n$. Итак, множество $Y$ ограничено в $\mathbb{N}$. Поэтому найдется наименьшее натуральное число, не принадлежащее $Y$, т.е. фактически наименьшее нестандартное натуральное $y$. Тогда $x=y-1-$ стандартное число. Значит, $y=x+1$ также стандартно, согласно Переносу, противоречие.

(ii) По доказанному, непустая st- $\in$-определимая совокупность $\{n \in \mathbb{N}: \neg$ st $n\}$ не содержит наименьшего элемента, т.е. принцип индукции в st- $\in$-языке не верен в $\mathbb{N}$. Что касается области $\mathbb{N} \cap \mathbb{S}$, допустим, что $Z=\{x \in \mathbb{N}: \varphi(x) \wedge$ st $x\} \neq \varnothing$.

\footnotetext{
${ }^{6}$ Более того, как показано в [16], BST конечно аксиоматизируется над ZFC в $\in$-языке.
} 
( $Z$ не обязательно множество.) Стандартизация дает нам стандартное множество $X \subseteq \mathbb{N}$ такое, что $X \cap \mathbb{N}=Z \cap \mathbb{N}$. Наименьший элемент $X$ будет наименьшим и в $Z$.

(iii) Если $x, y \in \mathbb{S}$, то $\langle x, y\rangle \in \mathbb{S}$ по Переносу. Однако по определению мы имеем $\left\langle x_{1}, \ldots, x_{n}, x_{n+1}\right\rangle=\left\langle\left\langle x_{1}, \ldots, x_{n}\right\rangle, x_{n+1}\right\rangle$, что позволяет провести доказательство утверждения индукцией по $n \in \mathbb{N} \cap \mathbb{S}$.

(iv) По Переносу, число \# $x$ элементов $x$ принадлежит $\mathbb{N} \cap \mathbb{S}$. Поэтому можно рассуждать индукцией по $n=\# x$. Для $n=0$ все очевидно. Пусть $\# x=n+1$. Перенос приносит элемент $a \in x \cap \mathbb{S}$, и множество $y=x \backslash\{a\}$ снова стандартно. Однако $\# y=n$, так что $y \subseteq \mathbb{S}$ по индуктивному предположению.

(v) Достаточно применить Базовую Идеализацию для $A_{0}=x$ и $\psi(x):=x$.

(vi) По Ограниченности, $x \subseteq s$ для некоторого стандартного $s$. Стандартизация приносит стандартное множество $q \subseteq \mathscr{P}(s)$ такое, что $y \in q \Leftrightarrow y \subseteq x$ для каждого стандартного $y$. Тем самым, $\widehat{x}=\bigcup(q \cap \mathbb{S})$. Положим $\psi(a)=x \backslash a$ для $a \in q$. Если $A \subseteq q$ стандартно и конечно, то $\bigcup A \in \mathbb{S}$ по Переносу, а потому $\bigcup A \in q$, значит, $\bigcup A \varsubsetneqq x$, так как $x$ нестандартно, и, наконец, $\bigcap_{a \in A} \psi(a) \varsubsetneqq x$. Остается применить Базовую Идеализацию для $\psi$ и $A_{0}=q$. Лемма 1.14 доказана.

1g. Абсолютно насыщенное расширение стандартного универсума. Оказывается, что теория BST вполне адекватно описывает абсолютно насыщенные расширения универсума ZFC! Понятно, что универсум $\mathfrak{V}=\langle\mathbf{V} ; \epsilon\rangle$ всех множеств - почти универсальная структура в ZFC, а потому, по теореме 1.13 , существует элементарное и ограниченное вложение $a \mapsto^{*} a$ универсума $\mathfrak{V}$ в некоторую абсолютно насыщенную структуру, скажем, $\left\langle{ }^{*} \mathbf{V} ;{ }^{*} \in\right\rangle$. Последняя удовлетворяет ZFC из-за элементарности. Дополнительно к этому вводится отношение стандартности на ${ }^{*} \mathbf{V}$, т.e. st $x$, когда $\exists a\left(x={ }^{*} a\right)$. Мы получаем структуру ${ }^{*} \mathfrak{V}=\left\langle{ }^{*} \mathbf{V} ;{ }^{*} \in\right.$, st $\rangle$.

ОПРЕДЕЛЕНИЕ 1.15. Нестандартной структурой или st- $\in$-структурой называется всякая структура вида $\mathfrak{X}=\left\langle X ; \epsilon_{X}, \mathrm{st}_{X}\right\rangle$, где $\epsilon_{X}-$ бинарное, a st ${ }_{X}-$ унарное отношение на множестве или классе $X$. Инъекция $\pi: Y \rightarrow X$ называется вложением $\mathbb{S}$-типа $\in$-структуры $\mathfrak{Y}=\left\langle Y ; \epsilon_{Y}\right\rangle$ в st- $\in$-структуру $\mathfrak{X}=$ $\left\langle X ; \in_{X}\right.$, st $\left._{X}\right\rangle$, когда образ $\operatorname{ran} \pi=\{\pi(y): y \in Y\}$ совпадает со стандартной частъю $(\mathbb{S})^{\mathfrak{X}}=\left\{x \in X: \mathrm{st}_{X} x\right\}$ структуры $\mathfrak{X}$, и мы имеем $y \in_{Y} y^{\prime} \Leftrightarrow \pi(y) \in_{X} \pi\left(y^{\prime}\right)$ для всех $y, y^{\prime} \in Y$.

В этом случае st- $\in$-структура $\mathfrak{X}$ будет называться расширением $\mathbb{S}$-тuпa структуры $\mathfrak{Y}$ (посредством отображения $\pi$ ).

TEOPEMA 1.16 .

(i) Доказуемо в ZFC, структура ${ }^{*} \mathfrak{V}=\left\langle{ }^{*} \mathbf{V} ;{ }^{*} \in\right.$, st $\rangle$ удовлетворяет BST и является элементарным расширением $\mathbb{S}$-типа (в смысле определения 1.15) структуры $\mathfrak{V}=\langle\mathbf{V} ; \in\rangle$ в смысле отображения $a \mapsto^{*} a$.

(ii) Теория BST есть st-консервативное расширение ZFC, в том смысле, что любал $\in$-формула ч выводима в ZFC тогда и только тогда, когда $\varphi^{\text {st }}$ выводима в BST. ${ }^{7}$ Следовательно, BST и ZFC равнонепротиворечивы.

\footnotetext{
${ }^{7}$ Отсюда, по Переносу, следует, что любая $\in$-формула $\varphi$ одновременно выводима в BST и в ZFC - это другая форма консервативности. Форма, приведенная в (ii), носит более
} 
ДокАЗАТЕЛЬСтво. (i) Точное содержание этого утверждения состоит в следующем. Доказательство теоремы 1.13 (вместе со всем вспомогательным материалом) может быть представлено как построение трех $\in$-формул, определяющих область ${ }^{*} \mathbf{V}$, отношение ${ }^{*} \in$ и элементарное вложение $a \mapsto{ }^{*} a$ универсума $\mathbf{V}$ в ${ }^{*} \mathbf{V}$ - следовательно, также и предикат стандартности st, вместе с доказательством, в ZFC, всех требуемых утверждений, например, что вложение действительно элементарно. Последнее означает, что выполнено $\varphi\left(x_{1}, \ldots, x_{n}\right) \Leftrightarrow$ $\varphi^{\left.{ }^{*} \mathbf{V} ;{ }^{*} \in\right\rangle}\left({ }^{*} x_{1}, \ldots,{ }^{*} x_{n}\right)$ (т.е. это доказано в $\mathbf{Z F C ~ - ~ в ~ с у щ н о с т и , ~ с л е д с т в и е м ~ 1 . 8 ) ~}$ для любой $\in$-формулы $\varphi$. Правая часть последней эквивалентности представляет собой также $\in$-формулу, с переменными $x_{1}, \ldots, x_{n}$, полученную очевидным образом из $\varphi$ и тех $\in$-формул, которые определяют ${ }^{*} \mathbf{V},{ }^{*} \in, x \mapsto{ }^{*} x$. Содержанием же теоремы 1.16 является доказательство $\varphi^{*} \mathfrak{V}$ (а это тоже определенная $\in$-формула) в ZFC для любой аксиомы $\varphi$ теории BST.

Мы, конечно, не будем рассуждать столь педантично. Схемы ZFC $\mathbf{Z C}^{\text {st }}$ и Переноса в $* \mathfrak{V}$ немедленно вытекают из того факта, что универсум всех множеств элементарно вкладывается в $\left\langle{ }^{*} \mathbf{V} ;{ }^{*} \in\right\rangle$, причем областью значений этого вложения $a{ }^{*} a$ является, по определению, стандартная часть универсума ${ }^{*} \mathfrak{V}$.

Аксиома Ограниченности вытекает из ограниченности этого вложения.

Докажем Стандартизацию. Пусть $X \in{ }^{*} \mathbf{V}$ стандартно, т.е. $X={ }^{*} a$ для некоторого множества $a$ в универсуме $\mathbf{V}$. Положим $b=\left\{c \in a: \Phi\left({ }^{*} c\right)^{*} \mathfrak{V}\right\}$. Тогда $Y={ }^{*} b$ удовлетворяет $\forall^{\text {st }} x(x \in Y \Leftrightarrow x \in X \wedge \Phi(x))$ в * $\mathfrak{V}$.

Наконец, Базовая Идеализация сводится к следующей задаче. Пусть $A_{0}$ и $X-$ множества в универсуме $\mathbf{V}$ и для каждого $a \in A_{0}$ задано $\psi(a) \in{ }^{*} \mathbf{V}$, причем * $\in$-пересечение $\bigcap_{a \in A} \psi(a)$ непусто для каждого конечного $A \subseteq A_{0}$ - а нужно доказать, что $* \in$-пересечение $\bigcap_{a \in A_{0}} \psi(a)$ непусто в $* \mathfrak{V}$. Но это следует из насыщенности. (На самом деле, приведение Базовой Идеализации к этой форме требует некоторых рассуждений, в частности, по выяснению природы стандартной конечности в *V. Мы предоставим это читателю, сославшись на $[25$; гл. 3] или статьи [21], [22].)

(ii) Допустим, что $\varphi^{\text {st }}$ выводима в BST. (Обратное направление очевидно.) Рассмотрим, рассуждая в ZFC, структуру * $\mathfrak{V}$. По теореме 1.16 , она удовлетворяет BST, поэтому $\varphi^{\text {st }}$ истинна в ${ }^{*} \mathfrak{V}$, т.е. $\varphi$ выполнена в стандартной части структуры $* \mathfrak{V}$. Но эта стандартная часть $\in$-изоморфна универсуму $\mathbf{V}$ через отображение $a \mapsto^{*} a$, так что $\varphi$ истинна в V. А это и означает доказательство $\varphi$ в ZFC. Теорема 1.16 доказана.

Применив теорему 1.16(i) в данной модели ZFC, мы выводим следующий результат.

СлеДСТвиЕ 1.17. Каждая модель теории ZFC вкладывается, как класс всех стандартных множеств, в некоторую модель BST.

ЗАмечАниЕ 1.18. Теорема 1.16(i) означает, что структура * $\mathfrak{V}$ представляет собой интерпретацию теории BST в ZFC, при которой (доказуемым в ZFC образом) осуществляется $\in$-изоморфизм между стандартной областью интерпретации и базовым "стандартным" универсумом (в данном случае универсумом ZFC).

общий характер, так как не все нестандартные теории включают соответствующую форму Переноса. 
Такие интерпретации уместно назвать интерпретациями $\mathbb{S}$-типа.

В менее формальных терминах, такую интерпретацию BST в ZFC можно понимать как расширение универсума ZFC до универсума BST, где первый остается классом всех стандартных множеств, - и все это на базе аксиом ZFC.

1h. Теория ограниченных множеств: приведение формул к $\Sigma_{2}^{\text {st }}$ виду. Важной особенностью теории BST является возможность приведения st- $\in$-формул к $\Sigma_{2}^{\text {st }}$-виду, т.е. к виду $\exists^{\text {st }} a \forall^{\text {st }} b$ ( $\in$-формула). Иными словами, сколь бы сложной ни была данная st- $\in$-формула, еe можно привести к эквивалентному, в BST, виду, содержащему ровно два вхождения предиката st через кванторы $\exists^{\mathrm{st}} a, \forall^{\mathrm{st}} b$.

ТЕОРЕмА 1.19 (приведение к $\Sigma_{2}^{\text {st }}$-виду). Пусть $\Phi\left(x_{1}, \ldots, x_{m}\right)$ - бесnараметрическая st- $\in$-ормула. Существует беспараметрическая $\in$-формула $\varphi\left(x_{1}, \ldots, x_{m}, a, b\right)$ такая, что следующая эквивалентность выводится в BST:

$$
\forall x_{1} \ldots \forall x_{m}\left(\Phi\left(x_{1}, \ldots, x_{m}\right) \Leftrightarrow \exists^{\text {st }} a \forall^{\text {st }} b \varphi\left(x_{1}, \ldots, x_{m}, a, b\right)\right) .
$$

ДокАЗАТЕЛЬство. Элементарные упрощения сводят теорему к случаю, когда $\Phi$ имеет предваренную форму ПЧ, где П - кванторный префикс, содержащий кванторы $\exists^{\text {st }} y, \forall^{\text {st }} y$ и $\exists y, \forall y$, а $\Psi$ - произвольная $\in$-формула. В этом предположении теорема доказывается в два шага.

Шаг 1 - это утверждение теоремы в случае, когда префикс П содержит только кванторы вида $\exists^{\text {st }} y, \forall^{\text {st }} y$. Пусть, к примеру, $\Phi(x)$ есть $\forall^{\text {st }} y \exists^{\text {st }} z \Psi(y, z, x)$. Для любого $u$ положим mon $u=\cap(u \cap \mathbb{S})$ (монада $u$ - пересечение всех стандартных элементов $u$ ). Пусть Ult $u$ есть $\in$-формула, говорящая " $u$ - ультрафильтр". Через $\vartheta(u)$ обозначим $\in$-формулу $\forall y \exists z \exists p \in u \forall \xi \in p \Psi(y, z, \xi)$. Эквивалентность

$$
\Phi(x) \Leftrightarrow \exists^{\text {st }} u(\text { Ult } u \wedge x \in \operatorname{mon} u \wedge \vartheta(u))
$$

влечет искомый результат, так как правая часть легко приводится к $\Sigma_{2}^{\text {st }}$-виду. Для доказательства (1) рассмотрим произвольное $x$. Возьмем, по Ограниченности, стандартное множество $d$, содержащее $x$, затем, по Стандартизации, стандартное $u \subseteq \mathscr{P}(d)$, удовлетворяющее $p \in u \Leftrightarrow x \in p$ для всех стандартных $p \in u$. По Переносу, $u$ - стандартный ультрафильтр над $d$ с $x \in$ mon $u$. Остается проверить, что

$$
\Phi(x) \Leftrightarrow \forall y \exists z \exists p \in u \forall \xi \in p \Psi(y, z, \xi)
$$

для каждого стандартного ультрафильтра $u$ с $x \in \operatorname{mon} u$. Пусть $u$ - такой ультрафильтр. Правая часть (2) равносильна $\forall^{\mathrm{st}} y \exists^{\mathrm{st}} z \exists p \in u \forall \xi \in p \Psi(y, z, \xi)$ согласно Переносу. Остается проверить, что, для любых стандартных $y, z$,

$$
\Psi(y, z, x) \Leftrightarrow \exists^{\mathrm{st}} p \in u \forall \xi \in p \Psi(y, z, \xi) .
$$

Однако как $d=\cup u$, так и $p=\{\xi \in d: \Psi(y, z, \xi)\}$ - стандартные множества по Переносу. Если при этом $p \in u$, то $x \in p$ и обе части (3) выполнены, а иначе они обе ложны, что и доказывает эквивалентность (3).

Шаг 2 - это индуктивный шаг $\exists y$ теоремы для предваренных формул вышеуказанного вида. Приведем к $\Sigma_{2}^{\mathrm{st}}$-виду формулу $\Phi(x):=\exists w \exists^{\mathrm{st}} a \forall^{\mathrm{st}} b \varphi(x, w, a, b)$, 
где $\varphi$ есть $\in$-формула. Это основано на следующей двойной эквивалентности:

$$
\begin{aligned}
\Phi(x) & \Leftrightarrow \exists^{\text {st }} a \exists^{\text {st }} W \exists w \in W \forall^{\text {st }} b \varphi(x, w, a, b) \Leftrightarrow \\
& \Leftrightarrow \exists^{\text {st }} a \exists^{\text {st }} W \forall^{\text {stfin }} B \exists w \in W \forall b \in B \varphi(x, w, a, b) .
\end{aligned}
$$

Первый переход здесь обеспечивается Ограниченностью. Для второго перехода нужно вывести, для любых стандартных множеств $a, W$ и любого $x$, что

$$
\forall^{\text {stfin }} B \exists w \in W \forall b \in B \varphi(x, w, a, b) \Leftrightarrow \exists w \in W \forall^{\text {st }} b \varphi(x, w, a, b) .
$$

Сразу применить Базовую Идеализацию нельзя, надо ограничить квантор по $b$. Считая $x$ и стандартные множества $a, W, d(x \in d)$ фиксированными, положим

$$
z_{b}=\{\langle y, w\rangle \in d \times W: \varphi(y, w, a, b)\} \quad \text { для любого } b .
$$

Найдется множество $B_{0}$ такое, что $\forall b^{\prime} \exists b \in B_{0}\left(z_{b}=z_{b^{\prime}}\right)$. Благодаря Переносу, множество $B_{0}$ можно выбрать стандартным, и тогда $\forall^{\text {st }} b^{\prime} \exists^{\text {st }} b \in B_{0}\left(z_{b}=z_{b^{\prime}}\right)$. Но в этом случае правая часть (4) равносильна $\exists w \in W \forall^{\text {st }} b \in B_{0} \varphi(x, w, a, b)$, что позволяет свести (4) к Базовой Идеализации простыми рассуждениями. Теорема 1.19 доказана.

На этой теореме в определяющей степени основаны наиболее фундаментальные свойства универсума множеств BST. Вот одно из приложений.

ТЕОРема 1.20 (BST). (i) Выполнено Собирание, т.е. для любой st-є-формуль $\Phi(x, y)$ (возможно, с параметрами) имеет место следующее:

$$
\forall X \exists Y \forall x \in X(\exists y \Phi(x, y) \Rightarrow \exists y \in Y \Phi(x, y)) .
$$

(ii) Для любой st- $\in$-формулы $\Phi(x, y)$ (возможно, с параметрами) выполнено:

$$
\forall X \exists F\left(F-\text { бункиия } \wedge X=\operatorname{dom} F \wedge \forall^{\text {st }} x \in X[\exists y \Phi(x, y) \Rightarrow \Phi(x, F(x))]\right) .
$$

ДокАЗАТЕльство. Для экономии места мы приведем доказательство (i), но сошлемся на [21], [22], а также книгу [25; гл. 3] в отношении пункта (ii). Аксиома Ограниченности показывает, что вполне достаточно получить результат в виде

$$
\forall^{\text {st }} X \exists^{\text {st }} Y \forall x \in X\left(\exists^{\text {st }} y \Phi(x, y) \Rightarrow \exists^{\text {st }} y \in Y \Phi(x, y)\right) .
$$

Далее, согласно теореме 1.19 , можно считать, что $\Phi$ есть $\Sigma_{2}^{\text {st }}$-формула. В этом случае внешний квантор $\Phi$, скажем, $\exists^{\text {st }} a$, может быть соединен с квантором $\exists^{\text {st }} y$. Значит, мы можем, не ограничивая общности, считать, что $\Phi-$ даже $\Pi_{1}^{\text {st }}$ формула, т.е. формула вида $\forall^{\text {st }} b \varphi(x, y, b)$, где $\psi$ есть $\in$-формула.

И последнее упрощение. Если $\psi$ имеет параметры, скажем, множество $p$, то оно принадлежит некоторому стандартному $P$, и тогда, заменив $X$ на $X \times P$, мы легко сводим задачу к беспараметрическому случаю. Итак, требуется вывести

$$
\forall^{\text {st }} X \exists^{\text {st }} Y \forall x \in X\left(\exists^{\text {st }} y \forall^{\text {st }} b \varphi(x, y, b) \Rightarrow \exists^{\text {st }} y \in Y \forall^{\text {st }} b \varphi(x, y, b)\right),
$$

где $\varphi$ - беспараметрическая $\in$-формула. 
Положим $h_{y b}=\{x \in X: \varphi(x, y, b)\}$ для всех $y, b$ и далее, для каждого $y$, $h_{y}=\left\{h_{y b}: b \quad\right.$-любое множество $\}$. Тогда $h_{y} \subseteq \mathscr{P}(X)$ для всех $y$. Согласно Собиранию из $\mathbf{Z F C}^{\text {st }}$ (в $\in$-языке) и Переносу ${ }^{8}$, имеется стандартное множество $Y$ такое, что $\forall y^{\prime} \exists y \in Y\left(h_{y}=h_{y^{\prime}}\right)$. Чтобы доказать, что $Y$ удовлетворяет (6), возьмем $x \in X$ и любое стандартное $y$ такое, что $\forall^{\text {st }} b \varphi(x, y, b)$. По выбору $Y$ и Переносу, найдется стандартное же $y^{\prime} \in Y$, удовлетворяющее $h_{y}=h_{y^{\prime}}$. Остается доказать $\varphi\left(x, y^{\prime}, b^{\prime}\right)$ для каждого стандартного $b^{\prime}$. Однако, коль скоро $h_{y}=h_{y^{\prime}}$, по Переносу, найдется стандартное $b$ такое, что $h_{y b}=h_{y^{\prime} b^{\prime}}$. В то же время выполнено $\varphi(x, y, b)$ по выбору $y$, другими словами, $x \in h_{y b}$. Значит, $x \in h_{y^{\prime} b^{\prime}}$, т.е. $\varphi\left(x, y^{\prime}, b^{\prime}\right)$, что и требовалось. Теорема 1.20 доказана.

Закончим важным следствием метаматематического характера.

СлЕДСТВИе 1.21. Ко всякой замкнутой st- $\in$-формуле $\Phi$ найдется $\in$-формула $\Psi$ такая, что $\Phi \Leftrightarrow \Psi \Leftrightarrow \Psi^{\text {st }}$ доказуемо в $\mathbf{B S T}$.

ДоказАТЕЛЬство. Теорема 1.19 дает $\Sigma_{2}^{\text {st }}$-формулу $\Theta:=\exists^{\text {st }} a \forall^{\text {st }} b \quad \vartheta(a, b)$ (где $\vartheta$ является $\in$-формулой), эквивалентную $\Phi$ в BST. Тогда, согласно Переносу, $\in$-формула $\Psi:=\exists a \forall b \vartheta(a, b)$ удовлетворяет $\Theta \Leftrightarrow \Psi$ в BST.

Исторические и библиографические замечания к 1 . Материал пп. $1 \mathrm{a}-1$ с относится к классике логики и теории моделей, по которой можно указать исчерпывающую литературу, например, [48] в отношении основных понятий логики и [39] в отношении теории моделей. В частности, о понятии интерпретации (п. 1а) см. [48; 4.7], об инвариантной интерпретации см. [48; 9.10].

О насыщенных структурах (п. 1b) см. книгу [39], а в контексте нестандартного анализа см., например, [41], [44]-[46]. Абсолютно насыщенные (в смысле п. 1е) расширения всего универсума ZFC были впервые построены, несколько разными методами и в разных, но так или иначе выходящих за рамки ZFC предпосылках, в [10], [11] и в [8]. Построение в рамках теории ZF с аксиомой глобального выбора (т.е. в соответствии с наброском в начале п. 1d) дано в [21], [22]. Совместность аксиомы глобального выбора с ZF установлена в [49].

Редукция классов к множествам, описанная в замечании 1.6, приписывается Д. Скотту в [50]; см. также в [47; 9.10] замечание после соотношения (13).

Проблема "эффективного” построения насыщенных расширений (п. 1f) была в свое время предложена В.А. Успенским одному из авторов (В. Г. Кановею); она упоминается как нерешенная задача в [21], [22]. Для случая расширений вещественной прямой ее решение было получено в [24], а для случая насыщенных в любой мощности расширений всего универсума в книге [25; гл. 4]. Эта конструкция представлена выше в 1.9 .

Теория ограниченных множеств BST (п. 1f) была введена в [14], но неявно содержится в более ранних работах [10], [11]. Вообще, аксиоматические основания нестандартного анализа уходят корнями в исследования 1970-х годов, когда были предложены три разные аксиоматические схемы: теория внутренних множеств IST [8], альтернативная теория множеств AST [51], [52] и аксиоматические построения в [10], [11]. Более подробно об этом написано в предисловии к книге [25].

\footnotetext{
${ }^{8}$ Следует заметить, что ссылки на Перенос здесь и ниже в доказательстве возможны только в силу того, что $\varphi$ предполагается беспараметрической формулой.
} 
Теорема 1.16(i) доказана в [25; гл. 4]. Часть (ii) этой теоремы и следствие 1.17, которые здесь выведены как элементарные следствия 1.16(i), были получены другими методами в [14], в сущности, на основе аналогичных результатов из [8] для IST. Центральная теорема BST о приведении всех st- $\in$-формул к $\Sigma_{2}^{\text {st }}$-виду (теорема 1.19) была доказана в [14]. Теорема 1.20, вместе с рядом других теорем BST, также впервые была получена в [14]. См. об этом в [21], [22], а также в наиболее обстоятельной форме в книге [25; гл. 3].

Уместно сказать несколько слов о взаимоотношении теории BST с весьма популярной по историческим причинам теорией внутренних множеств IST Нельсона [8]. Разница между обеими теориями состоит в том, что аксиома Базовой Идеализации из BST усиливается в IST до следующей схемы полной идеализации (или интернализации, как в работах [41], [44]):

Идеализация: $\forall^{\text {stfin }} A \exists x \forall a \in A \Phi(a, x) \Leftrightarrow \exists x \forall^{\text {st }} a \Phi(a, x)$, где $\Phi(a, x)$ - любая $\in$-формула с произвольными параметрами.

Соответственно, аксиома Ограниченности из BST не включается в IST, так как Идеализация влечет существование множества, содержащего все стандартные множества, что противоречит Ограниченности. ${ }^{9}$ Итак, BST и IST несовместимы. В то же время нетрудно проверить, что класс $\left\{x: \exists^{\text {st }} y(x \in y)\right\}$ всех ограниченных множеств образует интерпретацию BST в IST.

Внутри универсума множеств теорий IST и BST можно образовать еще более узкие классы $\mathbb{I}_{\kappa}=\left\{x: \exists^{\mathrm{st}} y(\operatorname{card} y \leqslant \kappa \wedge x \in y)\right\}$, где $\kappa-$ фиксированный стандартный кардинал. В таком частично насыщенном подуниверсуме $\mathbb{I}_{\kappa}$ выполняются аксиомы теории BST после соответствующей модификации, в которой Ограниченность усилена требованием card $y \leqslant \kappa$, а Базовая Идеализация аналогичным образом ослаблена.

Для теории IST теорема 1.19 не имеет места: как установлено в [14], имеется st- $\in$-формула, не эквивалентная в IST никакой $\Sigma_{2}^{\text {st }}$-формуле. В то же время в [8], [9] показано, что в IST к $\Sigma_{2}^{\text {st }}$-виду приводятся формулы, все кванторы которых ограничены стандартными множествами (например, $\exists x \in X, \forall^{\text {st }} y \in Y$, где $X$ и $Y$ стандартны). Подробнее об этом см. книгу [25; гл. 3], где, кроме того, изложены результаты по частично насыщенным подуниверсумам типа $\mathbb{I}_{\kappa}$.

Различия между теориями BST и IST в их аксиоматиках и метаматематических свойствах никак не влияют на прикладные возможности обеих теорий как основы "нестандартных" рассуждений в разных областях математики, но самым серьезным образом сказываются в более глубоких вопросах оснований. Например, теорема 1.16(ii) сохраняет силу для IST, но следствие 1.17 уже не имеет места для нее (опровергается минимальными транзитивными моделями ZFC, как показано в [21]). Поэтому теория IST не допускает интерпретации в ZFC такого рода, как в теореме 1.16(i). Нетрудно понять, почему конструкции типа изложенных выше не могут привести к интерпретации IST в ZFC: учитывая неограниченный характер Идеализации, в ходе построения нам пришлось бы перебирать всевозможные подклассы (а не только подмножества) стандартного универсума, что невозможно выполнить в ZFC. В то же время, добавив к ZFC предикат истинности $\in$-формул, с соответствующими аксиомами, можно получить теорию, в которой IST интерпретируется. Об этом см. в [25; 4.6].

\footnotetext{
${ }^{9}$ Ограничив одну или обе переменные $x, a$ в формулировке Идеализация и стандартными множествами, мы получаем схемы, все еще доказуемые в BST, см. [25; 3.2].
} 


\section{§ 2. Присоединение классов к нестандартному универсуму}

Этот параграф посвящен нестандартным теориям классов. Начнем мы с обзора стандартных и нестандартных теорий классов Гёделя-Бернайса GB и nGB и Келли-Морса KM и nKM. Теории nGB и nKM формулируются соответственно как непредикативная и предикативная теории классов над нестандартным универсумом множеств теории BST. Метаматематические связи между стандартными и нестандартными теориями классов представлены в диаграммах на с. 90.

Главный результат, теорема 2.16, приносит "реалистическую" интерпретацию теорий $\mathbf{n G B}$ и $\mathbf{n K M}$ соответственно в $\mathbf{G B}$ и $\mathbf{K M}$.

Метод доказательства этой теоремы может быть истолкован как присоединение классов к нестандартному универсуму множеств BST и, таким образом, как решение проблемы 2 из введения. Это выглядит достаточно похоже на присоединение нестандартных множеств к стандартному универсуму ZFC с целью построения нестандартного универсума множеств BST в 1 , однако метаматематическая сторона несколько иная. Именно, в то время как средств ZFC было вполне достаточно для построения искомого BST-расширения по теореме 1.16, теперь средств BST уже недостаточно для присоединения классов даже в случае nGB. Причина этого проявляется уже для пары стандартных теорий ZFC, GB: вторая, консервативное и равнонепротиворечивое расширение первой, тем не менее не допускает интерпретацию в первой по теореме 2.2. Таким образом, BST-структуре здесь нужна некоторая помощь. В сущности, достаточно предположить, что стандартная область универсума BST уже расширена классами до универсума GB или $\mathbf{K M}$ - этого достаточно для построения нестандартной классовой оболочки всего универсума BST (см. замечание 2.20).

Однако фактически структура изложения будет состоять в том, что сначала, используя результаты $\S 1$, мы расширяем область множеств универсума GB (или $\mathbf{K M}$ ) до нестандартного универсума множеств, удовлетворяющего BST, а затем, рассуждая все еще в $\mathbf{G B}$ (или $\mathbf{K M}$ ), уже присоединяем классы к последнему.

2а. Стандартные теории классов. Предикативная теория классов Гёделя-Бернайса $\mathbf{G B}$ определяет своего рода "минимальное” расширение универсума множеств ZFC за счет классов - совокупностей множеств, которые сами не обязательно являются множествами. При этом множества - это по определению те классы, которые принадлежат, как элементы, другим классам. Классы, не являющиеся множествами, называются собственными классами. Итак, " $X-$ множество" выражается формулой set $X:=\exists Y(X \in Y)$.

Соответственно, для каждой $\in$-формулы $\varphi$ через $\varphi^{\text {sets }}$ обозначается ее релятивизация к множествам, т.е. все кванторы $\exists Y \ldots$ и $\forall Y \ldots$ в $\varphi$ меняются соответственно на $\exists Y(\operatorname{set} Y \wedge \ldots)$ и $\forall Y(\operatorname{set} Y \Rightarrow \ldots)$. Формулы вида $\varphi^{\text {sets }}$ называются предикативными, а непредикативная означает: не обязательно предикативная. Никаких ограничений на свободные переменные (параметры) здесь не налагается. Обычно соглашаются употреблять малые буквы только для обозначения множеств, а большие - для произвольных классов (множеств и собственных классов). Например, формула $\exists x \varphi(x)$ понимается как сокращение для $\exists X(\operatorname{set} X \wedge \varphi(X))$. 
ОПРЕДЕлЕНиЕ 2.1. Теория классов Гёделя-Бернайса GB включает:

$\mathbf{Z F C}^{\text {sets }}$ : все формулы вида $\varphi^{\text {sets }}$, где $\varphi$ - аксиома ZFC (в $\in$-языке);

Экстенсиональность: $\forall X \forall Y(\forall x(x \in X \Leftrightarrow x \in Y) \Leftrightarrow X=Y)$;

Свертка: $\exists X \forall x(x \in X \Leftrightarrow \Phi(x))$, где $\Phi$ - любая предикативная $\in$-формула ${ }^{10}$;

Пересечение: $\forall X \forall y$ (set $(X \cap y))$ : пересечение класса и множества - множество;

Собирание: $\forall P \forall d \exists r \forall x \in d(\exists y(\langle x, y\rangle \in P) \Rightarrow \exists y \in r(\langle x, y\rangle \in P))$.

Положим $C[x]=\{y:\langle x, y\rangle \in C\}$ (сечение) для любых класса $C$ и множества $x$.

Теория классов Келли-Морса KM определяется аналогично $\mathbf{G B}$, с той лишь разницей, что Свертка и Собирание усиливаются до следующих схем:

Непредикативная Свертка: $\exists X \forall x(x \in X \Leftrightarrow \Phi(x))$, где $\Phi(x)$ - любая (возможно, непредикативная) $\in$-формула; ${ }^{10}$

Собирание Классов: $\forall d \exists C \exists r \forall a \in d(\exists Y \Phi(a, Y) \Rightarrow \exists x \in r \Phi(a, C[x]))$, где $\Phi(x)$ - любая (возможно, непредикативная) $\in$-формула. ${ }^{10}$

Таким образом, $\mathbf{Z F C}^{\text {sets }}$ постулирует, что класс всех множеств удовлетворяет ZFC. Схемы Свертки утверждают, что любая совокупность множеств вида $\{x: \Phi(x)\}$ (Ф предикативна для GB) есть класс. Свертки, Пересечения и Собирания достаточно для вывода схем Выделения и Подстановки теории ZFC. Сама же (предикативная) Свертка выводится из некоторого конечного числа ее примеров (см., например, [35; гл. II, §6]). Отсюда следует, что теория классов GB, в отличие от $\mathbf{Z F C}$, конечно аксиоматизируема (и обычно явно формулируется с конечным списком аксиом).

Теорема 2.2. 1) Теория GB есть sets-консервативное расширение ZFC, в том смысле, что $\in$ формула $\varphi$ выводима в ZFC тогда и только тогда, когда $\varphi^{\text {sets }}$ - теорема GB. ${ }^{11}$ Следовательно, теории ZFC и GB равнонепротиворечивы.

2) Однако $\mathbf{G B}$ не имеет интерпретации в ZFC.

3) Теория KM даже не равнонепротиворечива с ZFC.

ДокАзАтельство. 1) Для вывода sets-консервативности допустим (нетривиальное направление), что $\in$-формула $\varphi$ не выводима в ZFC. Тогда в ZFC имеется модель $\langle M ; \varepsilon\rangle$ теории $\mathbf{Z F C}+\neg \varphi$. Добавим к $M$ все множества $X \subseteq M$, $\in$-определимые в $\langle M ; \varepsilon\rangle$ (параметры из $M$ разрешены), кроме множеств вида $\widehat{x}=\{y \in M: y \varepsilon x\}, x \in M$, удаляемых для сохранения Экстенсиональности. Продолжим $\varepsilon$ на эту расширенную область $K$ так, что $x \varepsilon X$ всякий раз, когда $x \in X \in K \backslash M$. Получилась модель $\langle K ; \varepsilon\rangle$ теории $\mathbf{G B}$, класс всех множеств которой тождествен $M$, так что формула $\varphi^{\text {sets }}$ ложна в $\langle K ; \varepsilon\rangle$, а потому невыводима в GB. ${ }^{12}$

\footnotetext{
103 десь и ниже в аналогичных случаях предполагается, что рассматриваемая формула (в данном случае $\Phi(x))$ может содержать параметры - множества или классы.

${ }^{11}$ Более сложный результат [49] состоит в том, что и GBGC, т.е. теория GB, усиленная аксиомой Глобального Выбора, $\exists F \forall y \neq \varnothing(F-$ функция $\wedge y \in \operatorname{dom} F \wedge f(y) \in y)$, которая постулирует существование глобальной функции выбора, - также sets-консервативное расширение ZFC.

${ }^{12}$ Таким образом, каждая модель $M \models \mathbf{Z F C}$ вкладывается, как класс всех множеств, в подходящую модель $K \models \mathbf{G B}$, причем все присоединяемые классы определимы в $M$, но само это GB-расширение неопределимо в $M$ согласно п. 2) теоремы 2.2 .
} 
2) Если бы GB имела интерпретацию в ZFC, то, в силу конечной аксиоматизируемости GB, искомые свойства интерпретации были бы выводимы из конечной подтеории $\mathbf{T} \subseteq \mathbf{Z F C}$ и сама ZFC была бы интерпретируема в $\mathbf{T}$. Однако известно, что ZFC влечет непротиворечивость Consis $\mathbf{T}$ всякой своей конечной подтеории $\mathbf{T}$, и мы быстро приходим к противоречию с теоремами Гёделя о неполноте.

3) Известно, что KM позволяет построить модель ZFC, даже доказать существование транзитивного множества $X$ такого, что $\langle X ; \epsilon\rangle$ - модель ZFC. (Дело в том, что Непредикативная Свертка позволяет построить предикат истинности для всех $\in$-формул, релятивизованных к множествам. См. [35; с. 159 и 76].) Тем самым, в KM доказывается формальная непротиворечивость ZFC, так что, по теоремам Гёделя о неполноте, KM не может быть равнонепротиворечивой с ZFC. Теорема 2.2 доказана.

Согласно sets-консервативности, GB доказывает о множествах только то, что ZFC доказывает для своего универсума множеств, - этот факт дает основания для достаточно либерального неформального обращения с классами в ZFC (см. п. 1а).

2b. Нестандартные теории классов. Идеология нестандартных теорий классов сводится к следующим двум общим принципам: 1) универсум множеств удовлетворяет удобной нестандартной теории множеств, например, BST; 2) Свертка принимается для st- $\in$-формул, а не только для $\in$-формул. Поскольку Выделение принято в BST только для $\in$-формул, отсюда с необходимостью следует, что, в отличие от стандартных теорий классов, подклассы множеств (полумножества, см. ниже) не обязательно являются множествами.

Примем для st- $\in$-языка соглашения п. 2a о формуле set, употреблении больших и малых букв, предикативных и непредикативных формулах.

ОПРЕДЕЛЕНИЕ 2.3. Нестандартная теория классов Гёделя-Бернайса nGB - это теория в st- $\in$-языке, включающая аксиомы Экстенсиональности и Собирания и схему Свертки, как в $\mathbf{G B}$, но, в отношении Свертки, для всех (предикативных) st- $\in$-формул $\Phi$, а также следующие аксиомы:

BST $^{\text {sets }}$ : все аксиомы BST, релятивизованные к универсуму всех множеств;

Стандартизация классов: $\forall X \exists^{\mathrm{st}} Y \forall^{\text {st }} z(z \in Y \Leftrightarrow z \in X)$;

Aксиома $\mathbf{S}=\mathbf{S}^{\text {loc }}: \forall X\left(\right.$ st $\left.X \Leftrightarrow \forall^{\text {st }} y \exists^{\text {st }} z(X \cap y=z)\right) .{ }^{13}$

Нестандартная теория классов Келли-Морса $\mathbf{n K M ~ о п р е д е л я е т с я ~ а н а л о - ~}$ гично, но

1) Свертка и Собирание усиливаются до Непредикативной Свертки и Собирания классов - как в KM, но для всех st- $\in$-формул $\Phi$, и

2) добавляются аксиомы (Непредикативная Свертка) $)^{\text {st }}$ и (Собирание классов) $)^{\text {st }}$, т.е. соответствующие аксиомы $\mathbf{K M}$ (для $\in$-формул $\left.\Phi\right)$, релятивизованные к области $\mathbf{S}=\{X:$ st $X\}$ всех стандартных классов (и множеств).

\footnotetext{
${ }^{13}$ Правая часть эквивалентности в скобках может быть названа свойством локалъной стандартности $X$. Таким образом, аксиома $\mathbf{S}=\mathbf{S}^{\mathrm{loc}}$, в нетривиальном направлении $\Leftarrow$, утверждает, что локальная стандартность влечет стандартность. В принципе, эта аксиома может рассматриваться и как определение стандартности для собственных классов при условии, что стандартность в области множеств задана - см. доказательство теоремы 2.6.
} 
Таким образом, эти теории определяют свои универсумы как классовые надстройки (по типу $\mathbf{G B}$ или по типу $\mathbf{K M}$ ) над универсумом множеств BST.

Следующее определение вводит удобную систему обозначений для работы с нестандартными теориями классов (и множеств).

ОПРЕДЕЛЕНИЕ 2.4 (для нестандартных теорий классов). Множество $x$ называется внутренним, если оно принадлежит стандартному множеству, формально $\exists^{\text {st }} y(x \in y)$. Класс $X$ называется внутренним, если он имеет вид $C[x]=\{y:\langle x, y\rangle \in C\}$, где $C$ - стандартный класс, а $x$ - внутреннее множество. Под классами здесь понимаются все объекты универсума, множествами же считаются классы, принадлежащие другим классам, в соответствии с п. 2а. Положим

$$
\begin{array}{rlrl}
\mathbb{H} & =\{x: \operatorname{set} x\} & & \text { (все множества), } \\
\mathbb{S} & =\{x \in \mathbb{H}: \operatorname{st} x\} & & \text { (стандартные множества), } \\
\mathbf{S} & =\{X: \operatorname{st} X\} & & \text { (стандартные классы), } \\
\mathbb{I} & =\left\{x: \exists^{\text {st }} y(x \in y)\right\} & & \text { (все внутренние множества), } \\
\mathbf{E} & =\{X: X \subseteq \mathbb{I}\} & \text { (классы внутренних множеств), } \\
\mathbf{I} & =\{\text { все внутренние классы }\}, & \\
\mathbf{H} & =\{\text { все вообще классы }\} . &
\end{array}
$$

Внутренние классы - это важная промежуточная совокупность классов в теории nGB, включающая, например, все предикативно $\in$-определимые классы, см. лемму 2.5(vi). Все стандартные классы - также и внутренние, а с другой стороны, каждое внутреннее множество - это тоже внутренний класс, так как $C=\{\langle x, y\rangle ; y \in x\}$ - стандартный класс по лемме 2.5(v). Таким образом, в разумно аксиоматизированных теориях мы имеем

$$
\mathbb{S} \subseteq \mathbb{I} \subseteq \mathbb{H} \subseteq \mathbf{H}, \quad \mathbf{S} \subseteq \mathbf{I} \subseteq \mathbf{E} \subseteq \mathbf{H}, \quad \mathbb{S}=\mathbf{S} \cap \mathbb{H}, \quad \mathbb{I}=\mathbf{I} \cap \mathbb{H} .
$$

В то же время, например, в BST картина тривиализируется в том смысле, что $\mathbb{S}=\mathbf{S} \varsubsetneqq \mathbb{I}=\mathbf{I}=\mathbb{H}=\mathbf{E}=\mathbf{H}$ (ключевое равенство $\mathbb{I}=\mathbb{H}$ обеспечивается аксиомой Ограниченности, а аксиома Пары делает все объекты множествами). B теории nGB также происходит некоторая тривиализация: $\mathbb{I}=\mathbb{H}$, поскольку из-за $\mathbf{B S T}^{\text {sets }}$ все множества являются внутренними, и, соответственно, $\mathbf{E}=\mathbf{H}$ (все классы состоят из внутренних множеств). Следующая лемма приводит дальнейшие результаты.

ЛЕмма 2.5 (nGB).

(i) Если $X \in \mathbf{S}, w \in \mathbb{S}, w \cap \mathbb{S} \subseteq X$, mo $w \subseteq X$.

(ii) Каждый класс $X \in \mathbf{S}$ равен объединению своих стандартных подмножеств.

(iii) Для любого стандартного класса $X$ если $X \neq \varnothing$, то $X \cap \mathbb{S} \neq \varnothing$.

(iv) Для любых классов $X, Y \in \mathbf{S}$ если $X \cap \mathbb{S}=Y \cap \mathbb{S}$, mо $X=Y$.

(v) Если $\varphi(x)$ - предикативная формула с классами из $\mathbf{S}$ (включая множества из $\mathbb{S})$ в качестве параметров, то $X=\{x: \varphi(x)\}$ - стандартный класc. 
(vi) Если $\varphi(x)$ - предикативная формула с классами из I (включая множества из II) в качестве параметров, то $X=\{x: \varphi(x)\}$ - внутренний класc.

(vii) (Предикативный Перенос.) Если $\Phi$ - замкнутая предикативная формула со стандартными классами в роли параметров, то $\Phi \Leftrightarrow \Phi^{\mathrm{st}}$.

(viii) $\mathbf{G B}^{\text {st }}$, m.е. все аксиомы теории $\mathbf{G B}$, в $\in$-языке, релятивизованные $\kappa$ стандартной области $\mathbf{S}$.

(ix) (Предполагая $\mathbf{n K M}) \mathbf{K M}^{\mathrm{st}}$, m.е. все аксиомы $\mathbf{K M}$, релятивизованные $\kappa \mathbf{S}$.

ДокаЗАтельство. (i) Согласно аксиоме $\mathbf{S}=\mathbf{S}^{\text {loc}}$, мы имеем $u=w \cap X \in \mathbb{S}$. При этом $w \cap \mathbb{S}=u \cap \mathbb{S}$. Отсюда $w=u \subseteq X$ благодаря Переносу из BST $\mathbf{B S t s}^{\text {sets }}$. (ii) выводится из аксиомы $\mathbf{S}=\mathbf{S}^{\text {loc }}$. (iii), (iv) следуют из (ii) и Переноса из $\mathrm{BST}^{\text {sets }}$.

(v) Рассмотрим предикативную $\in$-формулу $\varphi(x)$ со стандартными классами $C_{1}, \ldots, C_{k}$ в роли параметров. Чтобы доказать, что $C=\{x: \varphi(x)\}$ - стандартный класс, зафиксируем множество $y \in \mathbb{S}$ и проверим, что $C \cap y \in \mathbb{S}$. Можно считать, что $\varphi$ записана в предваренной форме, т.е. как

$$
\mathrm{Q}_{1} v_{1} \ldots \mathrm{Q}_{n} v_{n} \psi\left(x, C_{1}, \ldots, C_{k}, v_{1}, \ldots, v_{n}\right),
$$

где $\psi$ - бескванторная $\in$-формула, в которую $C_{1}, \ldots, C_{k}$ входят лишь справа от $\in$.

Согласно Собиранию теории nGB и Ограниченности из BST $^{\mathrm{sets}}$, найдутся стандартные множества $r_{1}, \ldots, r_{n}$ такие, что для каждого $x \in y$ выполнено

$$
x \in C \Leftrightarrow \mathrm{Q}_{1} v_{1} \in r_{1} \ldots \mathrm{Q}_{n} v_{n} \in r_{n} \psi\left(x, C_{1}, \ldots, C_{k}, v_{1}, \ldots, v_{n}\right) .
$$

Однако множество $r=y \cup r_{1} \cup \cdots \cup r_{n}$ стандартно, а потому для каждого $i=1, \ldots, k \quad c_{i}=C_{i} \cap r$ принадлежит $\mathbb{S}$ по аксиоме $\mathbf{S}=\mathbf{S}^{\text {loc }}$. Теперь из (7) следует, что

$$
x \in C \Leftrightarrow \mathrm{Q}_{1} v_{1} \in r_{1} \ldots \mathrm{Q}_{n} v_{n} \in r_{n} \psi\left(x, c_{1}, \ldots, c_{k}, v_{1}, \ldots, v_{n}\right)
$$

для каждого $x \in y$, так что множество $C \cap y$ определяется $\in$-формулой со стандартными параметрами. Значит, $C \cap y \in \mathbb{S}$ благодаря Переносу из $\mathbf{B S T}^{\text {sets }}$.

(vi) По определению внутренних классов, можно считать, что $\varphi$ содержит только стандартные классы, а также любые множества (из II) в роли параметров. Пусть, скажем, $\varphi$ есть $\varphi(p, x)$, где $p \in \mathbb{I}$, а стандартные параметры не указаны явно. Тогда $C=\{\langle p, x\rangle: \varphi(p, x)\}$ - стандартный класс по (v). Но $C[p]=\{x: \varphi(p, x)\}$.

(vii) Достаточно проверить, что $\exists x \varphi(x) \Rightarrow \exists^{\text {st }} x \varphi(x)$ выполнено для любой $\in$-формулы $\varphi$ со стандартными параметрами. А это немедленно следует из (iii) и $(\mathrm{v})$.

(viii) $\left(\mathbf{Z F C}^{\text {sets }}\right)^{\text {st }}$ следует из BST ${ }^{\text {sets }}$. Релятивизации Экстенсиональности и Пересечения легко доказываются при помощи первых утверждений леммы. $(\text { Свертка })^{\text {st }}$ - это, в сущности, (v). Для вывода $\left(\right.$ Собирания) ${ }^{\text {st }}$ рассмотрим стандартные класс $P$ и множество $d$. Согласно Собиранию nGB и Ограниченности (из $\left.\mathbf{B S T}^{\mathrm{sets}}\right)$, найдется стандартное множество $r$ такое, что $\forall x \in d(\exists y P(x, y)$ $\Rightarrow \exists y \in r P(x, y))$. Остается применить Перенос из (vii). 
(ix) Немедленно следует из (viii), поскольку релятивизации двух ключевых аксиом $\mathbf{K M}^{\text {st }}$ введены в $\mathbf{n K M . ~ Л е м м а ~} 2.5$ доказана.

В метаматематическом плане, теории nGB и nKM связаны как с нестандартной теорией множеств BST, так и со стандартными теориями классов $\mathbf{G B}$ и KM. Разные аспекты связей nGB и $\mathbf{n K M}$ с теориями $\mathbf{G B}$ и $\mathbf{K M}$ рассматриваются ниже в этом параграфе, а основные взаимоотношения с BST даются следующей теоремой.

ТЕОРема 2.6. Теория nGB является sets-консервативным расширением BST в том же смысле, что и в теореме 2.2, но для всех st- $\in$-формул $\varphi,{ }^{14}$ a потому nGB равнонепротиворечива с $\mathbf{B S T}$ (и, по предыдущему, с ZFC). Однако nGB не допускает интерпретации в $\mathbf{B S T}$, а $\mathbf{n K M}$ даже не равнонепротиворечива с $\mathbf{B S T}$.

ДокАЗАТЕЛЬСтво. "Отрицательная" часть теоремы без труда выводится из теоремы 2.2, со ссылкой на теорему 1.16 для перехода от BST к ZFC. Для вывода консервативности достаточно проверить, что любая модель $\mathfrak{M}=$ $\langle M ; \varepsilon, \mathrm{st}\rangle \models \mathbf{B S T}(M-$ множество) может быть пополнена классами до модели $\mathfrak{K}=\langle K ; \varepsilon, \mathrm{st}\rangle \models \mathbf{n G B}$.

Мы получаем $K$, присоединяя к $M$ все множества $X \subseteq M$, st- $\in$-определимые в $\mathfrak{M}$ (параметры из $M$ разрешены), кроме множеств вида $\widehat{x}=\{y \in M: y \varepsilon x\}$, $x \in M$, причем $X \in K \backslash M$ объявляется стандартным в соответствии с аксиомой $\mathbf{S}=\mathbf{S}^{\text {loc }} .^{15}$ Проверка всех аксиом $\mathbf{n G B}$ в $\mathfrak{K}$ не составляет труда. Например, для вывода Собирания достаточно применить в $\mathfrak{M}$ теорему 1.20(i). Аксиома $\mathbf{S}=$ $\mathbf{S}^{\text {loc }}$ выводится в BST (нетривиальное направление использует лемму 1.14(vi)), и поэтому она верна в “старой” области $\mathfrak{M}$ (а в области $\mathfrak{K} \backslash \mathfrak{M}$ она верна по определению).

Несколько слов о Стандартизации классов. Прежде всего, из Стандартизации теории BST в $\mathfrak{M}$ следует, что предложение $\forall X \forall^{\text {st }} y \exists^{\text {st }} z(z \cap \mathbb{S}=X \cap y \cap \mathbb{S})$ верно в $\mathfrak{K}$. Остается вывести Стандартизацию классов из этого предложения. Пусть $X$ - любой класс. Положим $Y=\bigcup\{x \in \mathbb{S}: x \cap \mathbb{S} \subseteq X\}$. Понятно, что $X \cap \mathbb{S}=Y \cap \mathbb{S}$. Чтобы доказать, что $Y-$ стандартный класс, возьмем любое $y \in \mathbb{S}$. Тогда $Y \cap y=\bigcup\{x \in \mathbb{S}: x \cap \mathbb{S} \subseteq X \cap y\}=z$, где $z \in \mathbb{S}$ удовлетворяет $z \cap \mathbb{S}=X \cap y \cap \mathbb{S}$. Теорема 2.6 доказана.

2c. Аксиома непредикативного Переноса в теории Келли-Морса. Теории $\mathbf{n G B}$ и $\mathbf{n K M}$, как мы их сформулировали, не содержат явно Перенос. Конечно, BST ${ }^{\text {sets }}$ содержит эту аксиому для множеств, а лемма 2.5(vii) дает даже больше, но мы имеем в виду непредикативные формы.

Заметим, что непредикативный Перенос между стандартным универсумом (множеств и классов) $\mathbf{S}$ и всем универсумом классов вообще противоречит nGB, например, потому, что nGB влечет отрицание аксиомы Пересечения из GB. (Класс всех стандартных натуральных чисел - непустой ограниченный

\footnotetext{
${ }^{14} \mathrm{C}$. ниже более сильный результат - следствие 2.21 , кстати, и с альтернативным доказательством.

${ }^{15}$ Равносильное определение стандартности классов в $\mathfrak{k}$ : стандартны те $X \subseteq M$, которые $\in$-определимы в $\mathfrak{M}$ с $\mathfrak{M}$-стандартными параметрами. Равносильность следует из 3.2.4(4) в [25].
} 
подкласс $\mathbb{N}$ без наибольшего элемента, т.е. определенно не множество, см. лемму 1.14(i).) Корректная форма непредикативного Переноса апеллирует к внутренним классам.

В следующей теореме, как обычно, ${ }^{\text {st }}$ означает релятивизацию к стандартным множествам и классам. Аналогично, int будет обозначать релятивизацию к внутренним классам, т.е. к I. О множествах здесь можно не говорить, так как все они суть внутренние классы. Поэтому $\varphi \Leftrightarrow \varphi^{\text {int }}$ для предикативных $\epsilon$-формул $\varphi$.

По соображениям, которые будут ясны ниже, теорема доказывается для модифицированных теорий $\mathbf{n} \mathbf{K M}^{\circ}$ и $\mathbf{n G B}^{\circ}$, которые получаются из $\mathbf{n K M}$ и $\mathbf{n G B :}$

1) удалением Непредикативной Свертки и Собирания Классов из первой теории и Свертки и Собирания из второй,

2) присоединением $\mathbf{K M}^{\mathrm{st}}$ к первой теории и $\mathbf{G B}^{\mathrm{st}}$ ко второй,

3) присоединением к обеим теориям аксиомы, говорящей: $C[x]=\{y$ : $\langle x, y\rangle \in C\}-$ класс, каковы бы ни были множество $x$ и стандартный класс $C$.

Из леммы 2.5 (viii), (ix) следует, что $\mathbf{n K M} \Rightarrow \mathbf{n K M} \mathbf{M}^{\circ}$ и $\mathbf{n G B} \Rightarrow \mathbf{n G B}^{\circ}$.

ТЕОРема 2.7. (nKM $\left.{ }^{\circ}\right)$ Пусть $\Phi$ - замкнутая $\in$-формула со стандартнъми параметрами (множествами и классами). Тогда $\Phi^{\mathrm{int}} \Leftrightarrow \Phi^{\mathrm{st}}$.

$\left(\mathbf{n G B}^{\circ}\right)$ То же самое, но толъко для предикативных $\in$-формул $\Phi$.

Доказательство этой теоремы о переносе основано на лемме, для которой нам придется ввести еще одно обозначение.

ОПРЕДЕЛЕНиЕ 2.8. Для каждой $\in$-формулы $\varphi\left(v_{1}, \ldots, v_{n}\right)$ с переменными $v_{i}$ и стандартными параметрами (т.е. множествами из $\mathbb{S}$ и классами из $\mathbf{S}$ ), не указанными явно, ${ }^{\text {st }}\left\{\left\langle x_{1}, \ldots, x_{n}\right\rangle: \varphi\left(x_{1}, \ldots, x_{n}\right)\right\}$ обозначает единственный (по $\mathbf{G B}^{\text {st }}$ или $\left.\mathbf{K M}^{\text {st }}\right)$ класс $X \in \mathbf{S}$, удовлетворяющий

$$
X=\left\{\left\langle x_{1}, \ldots, x_{n}\right\rangle: \varphi\left(x_{1}, \ldots, x_{n}\right)\right\} \quad \text { в } \quad \mathbf{S},
$$

т.e.

$$
\left\langle x_{1}, \ldots, x_{n}\right\rangle \in X \Leftrightarrow \varphi\left(x_{1}, \ldots, x_{n}\right)^{\text {st }} \quad \text { для всех стандартных } x_{1}, \ldots, x_{n} .
$$

Лемма 2.9. (nKM $\left.{ }^{\circ}\right)$ Пусть $\varphi\left(v_{1}, \ldots, v_{n}\right)$ является $\in$-формулой со стандартными параметрами (множествами и классами). Тогда для всех множеств $x_{1}, \ldots, x_{n}$

$$
\varphi\left(x_{1}, \ldots, x_{n}\right)^{\mathrm{int}} \Leftrightarrow\left\langle x_{1}, \ldots, x_{n}\right\rangle \in{ }^{\mathrm{st}}\left\{\left\langle\xi_{1}, \ldots, \xi_{n}\right\rangle: \varphi\left(\xi_{1}, \ldots, \xi_{n}\right)\right\} .
$$

$\left(\mathbf{n G B}^{\circ}\right)$ То же для предикативных $\in$-формул $\varphi$.

ДоКАЗАТЕЛЬСТво. Используем индукцию по логической сложности формулы $\varphi$. Пусть $\varphi$ - атомарная формула $v_{1} \in v_{2}$. Тогда $X={ }^{\text {st }}\{\langle x, y\rangle: x \in y\} \in \mathbf{S}$. Согласно Ограниченности из $\mathbf{B S T}^{\mathrm{sets}}$, достаточно доказать для любого стандартного множества $z$, что $\forall x, y \in z(x \in y \Leftrightarrow\langle x, y\rangle \in p)$, где $p=X \cap(z \times z)-$ также стандартное множество по аксиоме $\mathbf{S}=\mathbf{S}^{\mathrm{loc}}$. Но это предложение истинно в $\mathbb{S}$ по определению $X$, так что остается использовать Перенос из $\mathbf{B S T}^{\text {sets }}$. 
Шаг для ᄀ. Стандартные классы $X={ }^{\mathrm{st}}\{x: \varphi(x)\}$ и $Y={ }^{\mathrm{st}}\{x: \neg \varphi(x)\}$ (точнее, их пересечения с классом $\mathbb{S}$ ) комплементарны в $\mathbb{S}$. Проверим, что $X$ и $Y$ комплементарны в II. Пусть, напротив, $z \in X \cap Y$. Лемма 2.5(ii) ${ }^{16}$ приносит стандартные множества $x, y$ такие, что $z \in x \subseteq X$ и $z \in y \subseteq Y$. Итак, $x \cap y \neq \varnothing$, откуда, по Переносу из $\mathbf{B S T}^{\mathrm{sets}}, x \cap y \cap \mathbb{S} \neq \varnothing$, а значит, $X \cap Y \cap \mathbb{S} \neq \varnothing$, противоречие.

Шаг для $\wedge$. Рассуждения вполне аналогичны: проверяем, для любых стандартных классов $X, Y, Z$, что $X \cap Y \cap \mathbb{S}=Z \cap \mathbb{S}$ влечет $X \cap Y=Z$.

Шаг для квантора $\exists$ по классам: наиболее сложный. Рассмотрим $\in$-формулу $\exists Y \varphi(v, Y)$ со стандартными параметрами. Пусть $X={ }^{\text {st }}\{\xi: \exists Y \varphi(\xi, Y)\}$. Нам нужно доказать, для любого $x$, эквивалентность $x \in X \Leftrightarrow(\exists Y \varphi(x, Y))^{\mathrm{int}}$. Согласно $\mathbf{B S T}^{\mathrm{sets}}, x$ принадлежит некоторому стандартному множеству $w$.

Слева направо. Пусть $x \in X$. По определению, мы имеем, в $\mathbf{S}, \forall \xi \in X \exists Y$ $\varphi(\xi, Y)$. Согласно (Собиранию Классов) ${ }^{\text {st }}$ из $\mathbf{n K \mathbf { M } ^ { \circ }}$, найдется класс $U \in \mathbf{S}$ такой, что в $\mathbf{S}$ выполнено $\forall \xi \in s \exists h \varphi(\xi, U[h])$, где $s=w \cap X \in \mathbb{S}$ и, напомним, $U[h]=\{x:\langle h, x\rangle \in U\}$. Однако $\varphi(\xi, U[h])$, как формула со свободными переменными $\xi, h$ (и $U$ в роли еще одного стандартного параметра), синтаксически проще, чем исходная формула $\exists Y \varphi(x, Y)$, поэтому по индуктивному предположению стандартный класс $P={ }^{\mathrm{st}}\{\langle\xi, h\rangle: \xi \in w \wedge \varphi(\xi, U[h])\}$ удовлетворяет $\langle\xi, h\rangle \in P \Leftrightarrow \xi \in w \wedge \varphi(\xi, U[h])^{\mathrm{int}}$.

Мы утверждаем, что $x \in \operatorname{dom} P$. В самом деле, поскольку $x \in X$, лемма 2.5(ii) приносит стандартное множество $u$ с $x \in u \subseteq X$. Можно считать, что $u \subseteq w$. (Иначе берем $w \cap u$ вместо $u ; w \cap u$ стандартно по Переносу из

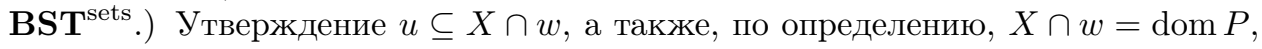
оба истинны в $\mathbf{S}$, поэтому найдется стандартное множество $p$ такое, что, в $\mathbf{S}$, $p \subseteq P$ и $u=\operatorname{dom} p$. Но тогда $p \subseteq P$ и $u=\operatorname{dom} p$ выполнены и в универсуме по лемме 2.5(i) для первого утверждения и Переносу из $\mathbf{B S T}^{\mathrm{sets}}$ для второго. Значит, $x \in u=\operatorname{dom} p \subseteq \operatorname{dom} P$.

Итак, $x \in \operatorname{dom} P$, т.е. найдется множество $h \mathrm{c}\langle x, h\rangle \in P$. Отсюда мы сразу получаем $\varphi(x, U[h])^{\mathrm{int}}$, поэтому $(\exists Y \varphi(x, Y))^{\mathrm{int}}$, так как $Y=U[h]-$ внутренний класс.

Справа налево. Предполагаем, что $\varphi(x, Y)^{\mathrm{int}}$, где $Y$ - внутренний класс. По определению, $Y=U[h]$, где $U$ - стандартный класс (не обязательно совпадающий с $U$, рассмотренным выше), а $h$ - множество. По индуктивному предположению, стандартный класс $P={ }^{\mathrm{st}}\{\langle\xi, \eta\rangle: \varphi(\xi, U[\eta])\}$ удовлетворяет $\langle\xi, \eta\rangle \in P \Leftrightarrow \varphi(\xi, U[\eta])^{\text {int }}$. Таким образом, $\langle x, y\rangle \in P$ и $x \in \operatorname{dom} P$. Тогда найдется стандартное $p \subseteq P$ с $x \in \operatorname{dom} p$ (лемма 2.5(ii)). Определяем, в $\mathbf{S}$, $u=\operatorname{dom} p$. Тогда $u \in \mathbb{S}$ и $u \subseteq X$ в $\mathbf{S}$ (так как по определению $X=\operatorname{dom} P$ в $\mathbf{S})$. Следовательно, $u \subseteq X$ и в универсуме по лемме 2.5(i). Наконец, согласно Переносу из $\mathbf{B S T}^{\mathrm{sets}}, u=\operatorname{dom} p$ выполняется в универсуме, так что $\operatorname{dom} p \subseteq X$. Отсюда $x \in X$, что и требовалось.

Шаг для квантора $\exists$ по множествам, для $\mathbf{n G B}^{\circ}$. Имея $\in$-формулу $\exists y \varphi(v, y)$ со стандартными параметрами, требуется доказать, что класс $X=$ st $\{\xi: \exists y \varphi(\xi, y)\}$ удовлетворяет $x \in X \Leftrightarrow \exists y \varphi(x, y)$ для любого $x$. Рассуждая аналогично шагу для квантора по классам, мы берем $P={ }^{\text {st }}\{\langle\xi, \eta\rangle: \xi \in$

\footnotetext{
16 Заметим, что утверждения (i)-(iv) леммы 2.5 в равной степени верны в $\mathbf{n G B}^{\circ}$.
} 
$w \wedge \varphi(\xi, \eta)\}$ для вывода слева направо и $P={ }^{\mathrm{st}}\{\langle\xi, \eta\rangle: \varphi(\xi, \eta)\}$ для вывода в обратном направлении. Лемма 2.9 доказана.

ДоКАЗАТЕЛЬСТВо тЕОРЕмы 2.7. Достаточно применить лемму $2.9 \mathrm{k} \varphi(v):=$ $(v=v \wedge \Phi)$, этим доказательство заканчивается.

СЛЕДСТВИЕ 2.10.

$\begin{array}{ll}\left(\mathbf{n K M} \mathbf{M}^{\circ}\right) & \text { Структура }\langle\mathbf{I} ; \in\rangle \text { удовлетворяет } \mathbf{K M} . \\ \left(\mathbf{n} \mathbf{G B}^{\circ}\right) & \text { Структура }\langle\mathbf{I} ; \in\rangle \text { удовлетворяет } \mathbf{G B} .\end{array}$

ДокАЗАТЕЛьство. Для $\mathbf{n K M}^{\circ}$-части просто применим Перенос (теорема 2.7) с учетом того, что $\langle\mathbf{S} ; \in\rangle$ удовлетворяет $\mathbf{K M}$ по аксиомам $\mathbf{K M}^{\text {st }}$. Для $\mathbf{n G B}{ }^{\circ}$-части некоторые аксиомы нужно проверить непосредственно, так как теорема 2.7 здесь дает Перенос только для предикативных формул. В частности, для проверки (предикативной) Свертки просто используем доказательство леммы 2.5(vi). Для проверки Собирания в $\langle\mathbf{I} ; \epsilon\rangle$ рассмотрим внутренний класс $P=C[p]$, где $p \in \mathbb{I}$, а $C$ - стандартный класс, а также множество $d \in \mathbb{I}$. Найдется стандартное множество $s$ с $p \in s$ и $d \subseteq s$. Согласно $\mathbf{G B}^{\text {st }}$, мы можем применить Собирание в $\mathbf{S}$, получая стандартное множество $c \subseteq C$, удовлетворяющее

$$
\forall q \in s \forall x \in s(\exists y(\langle x, y\rangle \in c[q]) \Rightarrow \exists y \in r(\langle x, y\rangle \in c[q]))
$$

в $\mathbb{S}$, где $r=\operatorname{ran} c$ - также стандартное множество. Остается отметить, что как включение $c \subseteq C$, так и (8) все еще истинны в $\mathbf{I}$ по теореме 2.7. Следствие 2.10 доказано.

2d. Аксиома $\Sigma_{2}^{\text {st }}$-классов. Отмеченные выше импликации $\mathbf{n K M} \Rightarrow \mathbf{n K M} \mathbf{M}^{\circ}$ и $\mathbf{n G B} \Rightarrow \mathbf{n} \mathbf{G B}^{\circ}$, вероятно, необратимы - однако они становятся обратимыми в присутствии следующей аксиомы, идея которой происходит из теоремы 1.19.

$\Sigma_{2}^{\text {st }}$-классы: Каждая совокупность вида $X=\left\{z: \exists^{\text {st }} x \forall^{\text {st }} y(\langle x, y, z\rangle \in I)\right\}$, где $I$ - внутренний класс, является классом, и наоборот, для любого класса $X$ найдется внутренний класс $I$ такой, что $X=\left\{z: \exists^{\text {st }} x \forall^{\text {st }} y(\langle x, y, z\rangle \in I)\right\} .{ }^{17}$

Насколько приемлема аксиома $\Sigma_{2}^{\text {st }}$-классов? Ответ на этот вопрос зависит, очевидно, от точки зрения на роль нестандартной теории классов. Если мы видим в системе классов своего рода минимальную оболочку универсума множеств и стандартных классов, то это как раз будет равносильно аксиоме $\Sigma_{2}^{\text {st }}$ классов.

TEOPEMA 2.11 .

$$
\begin{aligned}
& \left(\mathbf{n K M} \mathbf{M}^{\circ}+\Sigma_{2}^{\mathrm{st}} \text {-классы) } \text { Выполнены все аксиомы } \mathbf{n K M}\right. \text {. } \\
& \left(\mathbf{n G B}^{\circ}+\Sigma_{2}^{\mathrm{st}}-\text { классы) }\right. \text { Выполнены все аксиомы nGB. }
\end{aligned}
$$

ДокАЗАтельСтво. Начнем с проверки Непредикативной Свертки в КМ-случае. Некоторая неожиданность результата состоит в том, что достаточно специальная $\Sigma_{2}^{\text {st }}$-форма определения классов обеспечивает замкнутость их совокупности относительно всех st- $\in$-определений. Искомый результат вытекает из следующей леммы.

\footnotetext{
${ }^{17}$ Первая часть этой аксиомы, конечно, выводима в nGB.
} 
ЛЕмма 2.12 (nKM $\mathbf{M}^{\circ}+\Sigma_{2}^{\text {st }}$-классы). Для любой беспараметрической (б.n.) st- $\in$-бормуль $\Phi\left(X_{1}, \ldots, X_{m}, x_{1}, \ldots, x_{n}\right)$ имеется б.n. $\in$-бормула $\varphi\left(X_{1}, \ldots, X_{m}\right.$, $\left.x_{1}, \ldots, x_{n}, a, b\right)$ такая, что для всех множеств $x_{i}$ и стандартных классов $X_{j}$ выполнено

$$
\Phi\left(X_{1}, \ldots, X_{m}, x_{1}, \ldots, x_{n}\right) \Leftrightarrow \exists^{\mathrm{st}} a \forall^{\mathrm{st}} b \varphi\left(X_{1}, \ldots, X_{m}, x_{1}, \ldots, x_{n}, a, b\right)^{\mathrm{int}} .
$$

ДокАЗАТЕЛьство. Действуем по схеме из доказательства теоремы 1.19. Элементарные упрощения сводят общий случай к случаю, когда

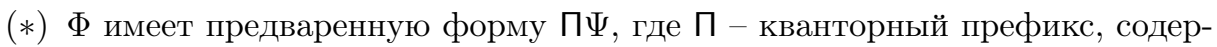
жащий кванторы только вида $\exists^{\text {st }} Y, \forall^{\text {st }} Y$ (по стандартным классам) и $\exists y, \forall y$ (по множествам), а $\Psi$ есть $\in$-формула (тогда $\Psi \Leftrightarrow \Psi^{\mathrm{int}}, \Phi \Leftrightarrow \Phi^{\mathrm{int}}$ ). В этом предположении лемма доказывается в два шага.

Шаг 1: утверждение леммы в случае, когда префикс П в $(*)$ содержит только кванторы вида $\exists^{\text {st }} Y, \forall^{\text {st }} Y$.

Пусть, к примеру, $\Phi(X, x)$ есть $\forall^{\text {st }} Y \exists^{\text {st }} Z \Psi(Y, Z, x, X)$. Через $\vartheta(u, X)$ обозначим $\in$-формулу $\forall Y \exists Z \exists p \in u \forall \xi \in p \Psi(Y, Z, \xi, X)$. В символике из доказательства теоремы 1.19 эквивалентность

$$
\Phi(X, x) \Leftrightarrow \exists^{\text {st }} u\left(\operatorname{Ult} u \wedge x \in \operatorname{mon} u \wedge \vartheta(u, X)^{\mathrm{int}}\right)
$$

влечет искомый результат, так как правая часть легко приводится к $\Sigma_{2}^{\text {st }}$-виду. Для доказательства (9) рассмотрим произвольное $x \in \mathbb{I}$ и стандартный класс $X$. Согласно Стандартизации из $\mathbf{B S T}^{\mathrm{sets}}$, существует стандартный ультрафильтр $u \in \mathbb{S}$ такой, что $x \in \operatorname{mon} u$ (см. доказательство теоремы 1.19). Остается показать, что

$$
\Phi(x, X) \Leftrightarrow \forall^{\text {int }} Y \exists^{\text {int }} Z \exists p \in u \forall \xi \in p \Psi(Y, Z, \xi, X)
$$

для каждого стандартного ультрафильтра $u$ с $x \in \operatorname{mon} u$. Вывод (10) совершенно аналогичен соответствующему рассуждению из доказательства теоремы 1.19 и опускается. (Перенос, используемый в выкладках, дается теоремой 2.7.)

Шаг 2: индуктивный шаг $\exists y$ леммы в предположениях (*). Преобразуем к (эквивалентному) $\Sigma_{2}^{\text {st }}$-виду формулу $\Phi(x, X):=\exists y \exists^{\mathrm{st}} a \forall^{\mathrm{st}} b \psi(y, a, b, x, X)^{\mathrm{int}}$, где $\psi$ есть $\in$-формула, а $X$ - переменная по стандартным классам. Утверждается, что

$$
\Phi(x, X) \Leftrightarrow \exists^{\mathrm{st}} a \exists^{\mathrm{st}} w \forall^{\mathrm{stfin}} \beta \exists y \in w \forall b \in \beta \psi(y, a, b, x, X)^{\mathrm{int}},
$$

- откуда немедленно следует искомый результат. Достаточно проверить, что

$$
\exists y \forall^{\text {st }} b \psi(y, b)^{\text {int }} \Leftrightarrow \exists^{\text {st }} w \forall^{\text {stfin }} \beta \exists y \in w \forall b \in \beta \psi(y, b)^{\text {int }}
$$

для любой $\in$-формулы $\psi$ со стандартными параметрами (множествами и классами). Импликация $\Rightarrow$ не составляет затруднений (по $\mathbf{B S T}^{\mathrm{sets}}$, стандартные конечные множества содержат только стандартные элементы, см. 3.1.20 в [25]), поэтому сосредоточимся на импликации $\Leftarrow$. Пусть стандартное $w$ обеспечивает правую часть (11). Согласно Переносу теоремы 2.7, класс $C=\left\{\langle y, b\rangle: \psi(y, b)^{\mathrm{int}}\right\}$ и отображение $b \mapsto w_{b}=\{y \in w: C(y, b)\}$ - стандартные классы. Собирание в $\langle\mathbf{S} ; \in\rangle$ и Перенос приносят нам стандартное множество $s$ такое, что $\forall b \exists b^{\prime} \in s$ 
$\left(w_{b}=w_{b^{\prime}}\right)$, и тогда $\forall^{\text {st }} b \exists^{\text {st }} b^{\prime} \in s\left(w_{b}=w_{b^{\prime}}\right)$. По выбору $w$, правая часть (11) влечет

$$
\forall^{\text {stfin }} \beta \subseteq s \exists y \in w \forall b \in \beta(\langle y, b\rangle \in c),
$$

где $c=C \cap(w \times s)$ (стандартное множество), и тогда $\exists y \in w \forall^{\mathrm{st}} b \in s\left(y \in w_{b}\right)$ по Базовой Идеализации из $\mathbf{B S T}^{\mathrm{sets}}$. (Заметим, что $y \in w_{b}$ равносильно $\langle y, b\rangle \in c$ при $y \in w$ и $b \in s$.) Пусть $y \in w$ удовлетворяет $\forall^{\text {st }} b \in s\left(y \in w_{b}\right)$. Тогда $\forall^{\text {st }} b$ $\left(y \in w_{b}\right)$ по выбору $s$, так что мы имеем левую часть (4), что и требовалось. Лемма 2.12 доказана.

Теперь о (предикативной) Свертке в предположении $\mathbf{G G B}^{\circ}$. Перенос теоремы 2.7 выполнен в этом случае только для предикативных $\in$-формул. Соответственно, лемма 2.12 сохраняет силу тоже только для предикативных st$\in$-формул, т.е. насколько позволяет доступная в этом случае предикативная форма Переноса. (Кванторы по стандартным классам на шаге 1 при этом, конечно, не появляются.) Но этого как раз вполне достаточно для вывода (предикативной) Свертки теории nGB.

Наконец, доказательство Собирания, или Собирания Классов, если предполагается $\mathbf{n} \mathbf{K M}^{\circ}$, вполне аналогично доказательству теоремы 1.20. Теорема 2.11 доказана.

2е. Полумножества и аксиома $\Sigma_{2}^{\text {st }}$-полумножеств. Здесь мы рассматриваем несколько дополнительных аксиом для нестандартных теорий классов, связанных с понятием полумножества. Эти аксиомы не входят в непосредственный круг идей, связанных с образованием подходящей оболочки из классов для нестандартного универсума множеств BST. Однако необходимость в этих дополнительных аксиомах быстро обнаруживается в приложениях.

ОПРЕДЕЛЕНИЕ 2.13. В нестандартных теориях классов полумножеством называется всякий подкласс множества. Данная st- $\in$-формула называется $n$ мограниченной (пм от "полумножество"), если каждый ее квантор по классам имеет вид $\exists^{\text {пм }}$ или $\forall^{\text {пм }}$ ("существует полумножество", "для всякого полумножества").

Например, если $p \in \mathbb{I}$ - функция (множество), определенная на множестве вида $a \times b$, где $a, b \in \mathbb{S}$, то, в теории $\mathbf{n G B}$, подкласс

$$
E_{p}=\left\{z: \exists^{\mathrm{st}} x \in a \forall^{\mathrm{st}} y \in b(z \in p(x, y))\right\}
$$

множества $r=\operatorname{ran} p \in \mathbb{I}$ является полумножеством. Обозначим через $\mathbf{C}_{0}$ класс всех $p \in \mathbb{I}$ указанного вида (коды $\Sigma_{2}^{\text {st }}$-полумножеств); $\mathbf{C}_{0} \subseteq \mathbb{I}$. Вводится аксиомa:

$\Sigma_{2}^{\text {st }}$-полумножества: для любого полумножества $X \subseteq \mathbb{I}$ найдется код $p \in \mathbf{C}_{0}$ такой, что $X=E_{p}$.

Полумножество $X$ имеет стандартный размер, когда найдутся стандартное множество $x$ и полумножество $F$, являющееся биекцией из $x \cap \mathbb{S}$ на $X$.

Каждое множество, конечно, является полумножеством (соответственно, предикативные формулы пм-ограничены), а в стандартных теориях классов верно и обратное, согласно аксиоме Пересечения. Аналог леммы 1.14(i) имеет место в $\mathbf{n G B}$, что приносит нам полумножество (стандартных 
натуральных чисел), не являющееся множеством. Что касается стандартного размера, заметим, что полумножество вида $x \cap \mathbb{S}$, где $x$ стандартно, это в сущности само $x$, рассматриваемое внутри универсума $\mathbb{S}$ стандартных множеств. (Бесконечные множества $x \in \mathbb{S}$ имеют и нестандартные элементы.) Стандартный размер свидетельствует о малости: подкласс полумножества стандартного размера сам является таковым. ${ }^{18}$

ЛЕмма 2.14 (nGB). Аксиома $\Sigma_{2}^{\mathrm{st}}$-классов влечет аксиому $\Sigma_{2}^{\mathrm{st}}$-полумножеств.

ДокАЗАТЕльство. Согласно $\Sigma_{2}^{\text {st }}$-классам, найдется внутренний класс $I$, для которого $X=\left\{z: \exists^{\text {st }} x \forall^{\text {st }} y(\langle x, y, z\rangle \in I)\right\}$. Собирание приносит множества $a, b \in \mathbb{S}$ такие, что $X=\left\{z: \exists^{\text {st }} x \in a \forall^{\text {st }} y \in b(\langle x, y, z\rangle \in I)\right\}$. Искомый код $p$ дается равенством $p(x, y)=\{z \in w:\langle x, y, z\rangle \in I\}$, где $w \in \mathbb{S}$ - любое множество с $X \subseteq w$. Лемма 2.14 доказана.

Аксиома $\Sigma_{2}^{\text {st }}$-полумножеств представляется менее ограничительной в контексте общих законов устройства нестандартных универсумов; она влечет ряд дальнейших следствий. Для дальнейшего нам удобно ввести такие две теории:

$$
\begin{array}{cc}
\mathbf{n G B}^{+}: & \mathbf{n G B}+\Sigma_{2}^{\mathrm{st}} \text {-полумножества - подтеория теории } \mathbf{n G B}+\Sigma_{2}^{\mathrm{st}} \text {-классы; } \\
\mathbf{n K M} & \mathbf{n K M}+\Sigma_{2}^{\mathrm{st}} \text {-полумножеств - подтеория теории } \mathbf{n K M}+\Sigma_{2}^{\mathrm{st}} \text {-классы. }
\end{array}
$$

Теория $\mathbf{n G B}^{+}$замечательна тем, что она включает, согласно следующей теореме, определенную часть схем Свертки и Собирания для непредикативных формул, оставаясь все еще $\mathbf{G B}$-теорией в смысле равнонепротиворечивости по теореме 2.16 .

Теорема 2.15 (nGB $^{+}$). Выполнены следующие предложения.

пм-ограниченная Свертка: Свертка для всех пм-ограниченных st- $\in$-формул;

Собирание полумножеств: $\forall d \exists^{\text {пм }} C \exists r \forall a \in d \quad\left(\exists^{\text {пм }} Y \varphi(a, Y) \Rightarrow \exists p \in r\right.$ $\varphi(a, C[p]))$, где $\varphi(a, Y)$ - любая пм-ограниченная st- $\in$-формула, а $C[p]$ введено в определении 2.1 .

Выбор стандартного размера: если $X$ - полумножество стандартного размера, а $P$ - любое полумножество, то существует множество-функиия $f$ такая, что $X \subseteq \operatorname{dom} f u$, для любого $x \in X, \exists y(\langle x, y\rangle \in P) \Rightarrow\langle x, f(x) \in P$.

Насыщенность: если $H$ есть П-замкнутое (т.е. $x, y \in H \Rightarrow x \cap y \in H$ ) полумножество стандартного размера $и \varnothing \notin H$, то пересечение $\bigcap H$ непусто.

Зависимый выбор: если $X$ - любое множество, $a R \subseteq X \times X-$ полумножество, причем $\operatorname{dom} R=X$, то найдется полумножество, имеющее вид последовательности $\left\{x_{n}\right\}_{n \in \mathbb{N} \cap \mathbb{S}}$, где $\left\langle x_{n}, x_{n+1}\right\rangle \in R$ для всех стандартных $n \in \mathbb{N}$.

Если дополнительно предполагается $\mathbf{n K M}$, то Собирание полумножеств распространяется на все, не обязательно пм-ограниченные, st- $\in$-формулы.

\footnotetext{
18 Зесь можно также требовать, чтобы не-малое множество содержало подмножество произвольно большого стандартного размера. В теории $\mathbf{n G B}+$ аксиома $\Sigma_{2}^{\text {st }}$-классов это требование также выполнено. См. об этом требовании в несколько иной ситуации в [25; $1.4]$.
} 


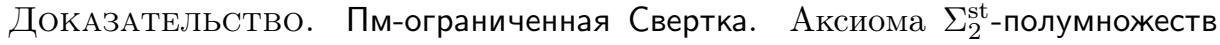
позволяет преобразовать любую пм-ограниченную формулу в предикативную: меняем каждое вхождение, например, $\exists^{\text {пм }} C \psi(C)$ на $\exists p \in \mathbf{C}_{0} \psi\left(E_{p}\right)$.

Выбор стандартного размера. По определению найдутся полумножествофункция $F$ и стандартное множество $d$ такие, что $F$ отображает $d \cap \mathbb{S}$ на $X$. По уже доказанной аксиоме $\Sigma_{2}^{\text {st }}$-полумножеств, $X, P, F$ st- $\in$-определимы в универсуме II всех множеств, с использованием параметров из $\mathbb{I}$. Теперь искомый результат легко получается при помощи теоремы 1.20(ii), применимой, так как I удовлетворяет BST.

Насыщенность. Согласно уже доказанному Выбору стандартного размера, найдутся стандартное множество $d$ и множество-функция $\psi$, отображающая $d \cap \mathbb{S}$ на $H$. Найдется и множество $R$ такое, что $\bigcup H \subseteq R$. Не ограничивая общности, предполагаем, что $\psi(a) \subseteq R$ для каждого $a \in d$. Из $\cap$-замкнутости $H$ следует, что для любого стандартного конечного множества $d^{\prime} \subseteq d$ пересечение $\bigcap_{a \in d^{\prime}} \psi(a)$ совпадает с некоторым $\psi\left(a^{\prime}\right), a^{\prime} \in d \cap \mathbb{S}$, а потому непусто. (Используется индукция по стандартному конечному числу элементов в $d^{\prime}$, см. лемму 1.14(ii).)

Зависимый выбор. Схема доказательства похожа на доказательство Выбора стандартного размера: сначала доказывается родственная теорема BST (см. [25; 3.2е]), к которой, при помощи аксиомы $\Sigma_{2}^{\text {st }}$-полумножеств, сводится искомый результат.

Собирание полумножеств. Формула $\varphi\left(a, E_{y}\right)$, с переменными $a, y$, по условию является пм-ограниченной. Поэтому, согласно пм-ограниченной Свертке (см. выше), существует класс $Z=\left\{\langle a, y\rangle: \varphi\left(a, E_{y}\right)\right\}$. Теперь обычное Собирание приносит множество $r$ такое, что $\exists p \varphi\left(a, E_{p}\right) \Rightarrow \exists p \in r \varphi\left(a, E_{p}\right)$ для каждого $a \in d$. Остается положить $C=\left\{\langle p, x\rangle: p \in r \wedge x \in E_{p}\right\}-$ тогда $C[p]=E_{p}$ для всех $p \in r$.

Если предполагается nKM, то для вывода Собирания полумножеств для произвольной st- $\in$-формулы $\varphi(a, Y)$ мы сначала применим Собирание Классов, которое приносит, для данного множества $d$, класс $C^{\prime}$ и множество $r$ такие, что

$$
\forall a \in d\left(\exists^{\text {пм }} Y \varphi(a, Y) \Rightarrow \exists p \in r\left(C^{\prime}[p]-\text { полумножество } \wedge \varphi\left(a, C^{\prime}[p]\right)\right)\right) .
$$

Согласно обычному Собиранию, найдется множество $Z$ такое, что, для каждого $a \in d$, если $C^{\prime}[p]$ - полумножество, то $C^{\prime}[p] \subseteq z$ для некоторого $z \in Z$. Теперь возьмем полумножество $C=C^{\prime} \cap(d \times \cup Z)$. Теорема 2.15 доказана.

\section{2f. Интерпретация нестандартных теорий классов в стандартных.} Целью этого пункта является доказательство следующей теоремы.

Tеорема 2.16. Существует интерпретация $\mathbf{S}-$ типа ${ }^{19}$ теорий $\mathbf{n G B}+\Sigma_{2}^{\mathrm{st}}$ классы $и \mathbf{n G B}^{+}$в $\mathbf{G B}$ (соответственно теорий $\mathbf{n K M}+\Sigma_{2}^{\mathrm{st}}$-классы $u \mathbf{n K} \mathbf{M}^{+}$ в $\mathbf{K M})$.

\footnotetext{
${ }^{19}$ Понятие об интерпретации и расширении $\mathbf{S}$-типа вполне аналогично определению 1.15 и замечанию 1.18 , т.е. речь идет о такой интерпретации, стандартная область $\mathbf{S}$ которой доказуемо изоморфна, через некоторое конкретное вложение, всему универсуму GB. Мы используем $\mathbf{S}$ вместо $\mathbb{S}$, поскольку вся стандартная область в теориях классов обозначается $\mathbf{S}$ согласно определению 2.4 .
} 
Теории $\mathbf{n G B}, \mathbf{n G B}^{+}$u $\mathbf{n G B}+\Sigma_{2}^{\text {st }}$-классы являются st-консервативными в смысле теоремы 1.16(ii) и, следовательно, равнонепротиворечивыми расширениями теории GB. Соответственно, теории $\mathbf{n K M}, \mathbf{n K M} \mathbf{M}^{+}$u $\mathbf{n K M}+\Sigma_{2}^{\mathrm{st}}-$ классы суть st-консервативные и равнонепротиворечивые расширения теории KM.

ДокАЗАТЕЛьство. Второе утверждение теоремы (о консервативности и равнонепротиворечивости) легко следует из первого, см., например, доказательство теоремы 1.16(ii), поэтому сосредоточимся на первом. Вполне достаточно ограничиться теорией $\mathbf{n G B}+\Sigma_{2}^{\text {st }}$-классы, поскольку $\mathbf{n G B}^{+}-$ее подтеория по лемме 2.14, и соответственно теорией $\mathbf{n K M}+\Sigma_{2}^{\text {st }}$-классы.

Рассуждая в $\mathbf{G B}$, мы обозначим через $\mathbf{K}$ универсум всех классов и через $\mathbf{V}=\{x: \operatorname{set} x\} \subseteq \mathbf{K}$ универсум всех множеств. Структура $\mathfrak{V}=\langle\mathbf{V} ; \in\rangle$ удовлетворяет ZFC, а потому мы можем провести, в $\mathbf{V}$, построение структуры ${ }^{*} \mathfrak{V}=\left\langle{ }^{*} \mathbf{V} ;{ }^{*} \in\right.$, st $\rangle$ и вложения ${ }^{*}: \mathbf{V} \rightarrow{ }^{*} \mathbf{V}$ таких, что ${ }^{*} \mathfrak{V}$ является абсолютно насыщенным нестандартным элементарным расширением $\mathfrak{V}$ посредством отображения *, см. п. $1 \mathrm{~g}$.

Напомним, что ${ }^{*} \mathfrak{V}$ удовлетворяет BST по теореме 1.16.

Чтобы избежать проблем в обозначениях, мы будем предполагать, что никакое множество $x \subseteq{ }^{*} \mathbf{V}$ не является элементом ${ }^{*} \mathbf{V}$; если это не так, то заменим каждое $x \in{ }^{*} \mathbf{V}$ парой $\langle x, x\rangle$ (а по определению класс ${ }^{*} \mathbf{V}$ не содержит ни одной упорядоченной пары) и переопределим отношения $* \in$, st и отображение * соответственно.

Итак, мы имеем интерпретацию $\mathbb{S}$-типа ${ }^{*} \mathfrak{V}$ теории BST в GB. Желая дополнить ее до интерпретации теории классов nGB, мы просто присоединим к ${ }^{*} \mathfrak{V}$ некоторые классы $X \subseteq{ }^{*} \mathbf{V}{ }^{20}$ Начнем с технического определения.

ОПРЕДЕЛЕНИЕ 2.17. Положим $\widehat{x}=\left\{y \in{ }^{*} \mathbf{V}: y^{*} \in x\right\}$ для каждого $x \in{ }^{*} \mathbf{V}$.

Класс $X \subseteq{ }^{*} \mathbf{V}$ назовем приемлемым, если он не имеет вида $\widehat{x}, x \in{ }^{*} \mathbf{V} .^{21}$

Положим ${ }^{*} A=\bigcup_{a \in \mathbf{V}, a \subset A}{ }^{*} a$ для любого собственного класса $A \in \mathbf{K}{ }^{22}$

Положим $\mathbb{I}={ }^{*} \mathbf{V}$; это переобозначение сделано для того, чтобы согласовать систему обозначений здесь с введенной в п. $2 \mathrm{~b}$.

Обозначим через $\mathbb{S}=\{x \in \mathbb{I}:$ st $x\}=\left\{{ }^{*} a: a \in \mathbf{V}\right\}$ стандартную область $* \mathfrak{V}$.

Мы рассмотрим следующие типы подклассов класса $\mathbb{I}={ }^{*} \mathbf{V}$.

$C$ пандартные классы: классы вида ${ }^{*} A$, где $A$ - любой собственный класс в $\mathbf{K}$.

Положим $\mathbf{S}=\mathbb{S} \cup\{$ все приемлемые стандартные классы $\}$.

\footnotetext{
${ }^{20}$ Неплохим упражнением для читателя будет понять, почему не имеет смысла присоединять все классы. Дело в том, что, вследствие абсолютной насыщенности * $\mathfrak{V}$, для многих элементов $x \in{ }^{*} \mathbf{V}$, например для любого $x={ }^{*} a$, где $a \in \mathbf{V}$ бесконечно, $\widehat{x}-$ собственный класс в смысле универсума GB. Приложив некоторые усилия, можно определить такой класс $F$, который является функцией из $\widehat{x}$ на конфинальный подкласс класса Ord всех ординалов. Присоединение к ${ }^{*} \mathfrak{V}$ класса $\left\{\left\langle x,{ }^{*} a\right\rangle:\langle x, a\rangle \in F\right\}$ приводит к неустранимому нарушению Собирания.

${ }^{21}$ Неприемлемые классы нельзя присоединять к * $\mathfrak{V}$ из-за противоречия с Экстенсиональностью.

22 Таким образом, *A для собственных классов определяется по-другому, чем для множеств (где определение ${ }^{*} a \in{ }^{*} \mathbf{V}$ для $a \in \mathbf{V}$ дается просто применением отображения ${ }^{*}$ ). Если мы все же определим, скажем, ${ }^{\star} x=\bigcup_{a \in \mathbf{V}}, a \subseteq x{ }^{* a}$ для некоторого множества $x$, то будет ${ }^{\star} x=\widehat{{ }^{\star} x} \neq{ }^{*} x$.
} 
Внутренние классы: классы вида $I=C[x]=\left\{y \in{ }^{*} \mathbf{V}:{ }^{*}\langle x, y\rangle \in C\right\}$, где $C$ - приемлемый стандартный класс, $x \in{ }^{*} \mathbf{V}, \mathrm{a}^{*}\langle x, y\rangle$ обозначает ${ }^{*} \mathfrak{V}$-nару, т.е. тот единственный элемент $p \in{ }^{*} \mathbf{V}$, для которого в $* \mathfrak{V}$ истинно, что $p$ есть пара $\langle x, y\rangle$. Положим $\mathbf{I}=\mathbb{I} \cup\{$ все приемлемые внутренние классы $\}$.

Внешние классы: классы вида $X=E_{I}=\bigcup_{x \in \mathbf{V}} \bigcap_{y \in \mathbf{V}} I\left[{ }^{*} x,{ }^{*} y\right]$, где $I$ - внутренний класс, $I[x, y]=\left\{z \in{ }^{*} \mathbf{V}:{ }^{*}\langle x, y, z\rangle \in I\right\}, \mathrm{a}^{*}\langle x, y, z\rangle \in{ }^{*} \mathbf{V}$ имеет тот же смысл, что ${ }^{*}\langle x, y\rangle$ выше. Положим $\mathbf{E}=\mathbb{I} \cup\{$ все приемлемые внешние классы $\}$.

Понятно, что совокупности $\mathbf{S}, \mathbf{I}, \mathbf{E}$ состоят из классов и множеств. Они рассматриваются в теориях классов на тех же основаниях, что и классы в ZFC см. п. 1а.

ОПредЕлЕНиЕ 2.18. Для $X, Y \in \mathbf{E}$ определим $X^{*} \in Y$, когда $X \in \mathbb{I}$ и либо $Y \in \mathbb{I}$ и $X^{*} \in Y$, либо $Y-$ приемлемый класс и просто $X \in Y{ }^{23}$

Положим st $X$ для каждого класса $X \in \mathbf{S}$.

Мы собираемся доказать, что структура $\mathfrak{E}=\left\langle\mathbf{E} ;{ }^{*} \in, \mathrm{st}\right\rangle$ удовлетворяет аксиомам теории $\mathbf{n G B}+\Sigma_{2}^{\text {st }}$-классы и является нестандартным (в смысле определения 1.15) расширением универсума $\mathbf{G B}$ посредством отображения *, а если дополнительно предполагается KM, то структура $\mathfrak{E}$ удовлетворяет и $\mathbf{n K M}$. Этим доказательство теоремы 2.16 будет закончено: структура $\mathfrak{E}$ образует "реалистическую" интерпретацию теории $\mathbf{n G B}+\Sigma_{2}^{\mathrm{st}}-$ классы в $\mathbf{G B}$ и теории $\mathbf{n K M}+\Sigma_{2}^{\text {st}}$-классы в $\mathbf{K M}$.

Утверждение о нестандартном расширении элементарно выводится из пунктов (i)-(iіi) следующей леммы.

ЛЕмма 2.19 (GB).

(i) $\operatorname{Eсли~} A \in \mathbf{K}, m o^{*} A \in \mathbf{S}$.

(ii) Если $X, Y \in \mathbf{K}$, mo $X \in Y \Leftrightarrow{ }^{*} X^{*} \in{ }^{*} Y$.

(iii) $\mathbf{S}=\{X \in \mathbf{E}:$ st $X\}=\left\{{ }^{*} A: A \in \mathbf{K}\right\}$ (стандартная область $\left.\mathfrak{E}\right) u \mathbb{S}=\mathbb{I} \cap \mathbf{S}$.

(iv) $\mathbb{I}=\left\{X \in \mathbf{E}:(\operatorname{set} X)^{\mathfrak{E}}\right\}=\{$ все множества в $\mathfrak{E}\}$.

(v) $\mathbf{I}=\{$ все внутренние классы в $\mathfrak{E}$ в смысле определения из $n .2 \mathrm{~b}\}$.

(vi) $B \mathfrak{E}$ истинна аксиома $\Sigma_{2}^{\text {st }}$-классов.

Доказательство. (i) Если $A=a-$ множество, то по определению * $a \in \mathbb{S} \subseteq$ S. Если $A$ - собственный класс, то достаточно проверить, что * $A$ - допустимый класс. Пусть, напротив, ${ }^{*} A=\widehat{x}=\left\{y \in \mathbb{I}: y{ }^{*} \in x\right\}, x \in \mathbb{I}$. Раз ${ }^{*} \mathfrak{V}=\left\langle\mathbb{I} ;{ }^{*} \in\right.$, st $\rangle$ удовлетворяет BST, найдется стандартное множество ${ }^{*} b \in \mathbb{I}(b \in \mathbf{V})$ такое, что $x \subseteq{ }^{*} b$ в ${ }^{*} \mathfrak{V}$. Отсюда следует ${ }^{*} A=\widehat{x} \subseteq{ }^{*} b$, и далее $a \subseteq b$ для любого $a \subseteq A$, т.е. $A \subseteq a$ и $A$ - множество (в "стандартной" $\mathbf{G B}$ ), что противоречит выбору $A$.

(ii) Считаем, что $X=x-$ множество (иначе обе части эквивалентности неверны). Если $Y$ - также множество, то используем тот факт, что * - элементарное отображение на множествах из V. Если $Y$ - собственный класс и $x \in Y$, то сразу ${ }^{*} x \in{ }^{*} Y$ (через посредство $a=\{x\}$ ). Допустим, что ${ }^{*} x \in{ }^{*} Y$, т.е. найдется множество $a \subseteq Y$ такое, что ${ }^{*} x \in{ }^{*} a$, т.е. ${ }^{*} x^{*} \in{ }^{*} a$. Тогда $x \in a \subseteq Y$, что и требовалось.

(iii) Здесь все просто: наше соглашение о том, что никакое $x \in \mathbb{I}={ }^{*} \mathbf{V}$ не может быть подмножеством II, устраняет все возможные недоразумения.

\footnotetext{
${ }^{23}$ Важно, что этим определением действие отношения $* \in$ на $\mathbb{I}={ }^{*} \mathbf{V}$ не меняется, поскольку в наших предположениях никакой класс $Y \subseteq \mathbb{I}$ не может быть элементом $\mathbb{I}$.
} 
(iv) По определению * $\in$-элементами в $\mathfrak{E}$ могут быть лишь множества из $\mathbb{I}$. $\mathrm{C}$ другой стороны, любое $x \in \mathbb{I}={ }^{*} \mathbf{V}{ }^{*} \in$-принадлежит некоторому $y \in \mathbb{I}$.

(v) Пусть $I \in \mathbf{E}-$ внутренний класс в $\mathfrak{E}$ в смысле п. $2 \mathrm{~b}$, т.е. найдутся класс $C \in \mathbf{S}$ и множество $x \in \mathbb{I}$ такие, что, в $\mathfrak{E}, I=\left\{y \in{ }^{*} \mathbf{V}:\langle x, y\rangle \in C\right\}$. Если $I \in \mathbb{I}$, то доказывать нечего, поскольку $\mathbb{I} \subseteq \mathbf{I}$. Поэтому предполагаем, что $I \subseteq \mathbb{I}$ - приемлемый класс. Если $C \in \mathbb{S}$, то $C$ - множество в $\mathbb{I}$, и тогда даже $I \in \mathbb{I}$. Если же $C$ - приемлемый стандартный класс, $C={ }^{*} A$ для некоторого собственного класса $A \in \mathbf{K}$, то по определению $I=C[x]-$ внутренний класс, что и требовалось.

(vi) Если $X \in \mathbb{I}$, то все ясно. Если $X$ - приемлемый внешний класс, то результат следует из определения внешних классов. Лемма 2.19 доказана.

Теперь следует проверка аксиом. Согласно теореме 2.11 и лемме 2.19(vi), достаточно вывести, что структура $\mathfrak{E}$ удовлетворяет аксиомам $\mathbf{n G B}^{\circ}-$ или даже $\mathbf{n} \mathbf{K M}^{\circ}$, если мы начинаем с теории $\mathbf{K M}$.

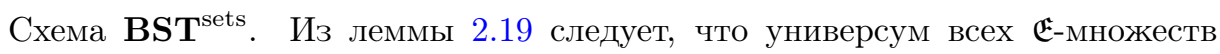
совпадает с $\left\langle\mathbb{I} ;{ }^{*} \in\right.$, st $\rangle$, но эта структура удовлетворяет BST.

Схемы $\mathbf{G B}^{\mathrm{st}}$, или даже $\mathbf{K M}^{\mathrm{st}}$, если предполагается $\mathbf{K M}$. Достаточно отметить, что, по лемме 2.19, стандартная область $\langle\mathbf{S} ; \in\rangle$ структуры $\mathfrak{E}$ изоморфна базовому "стандартному" универсуму $\mathbf{K}$ посредством отображения * .

Аксиома $\mathbf{S}=\mathbf{S}^{\text {loc }}$. Пусть $X={ }^{*} A \in \mathbf{S}\left(A-\right.$ собственный класс) и $y={ }^{*} a \in \mathbb{S}$ $(a \in \mathbf{V})$. Тогда $b=A \cap a-$ все еще множество в универсуме GB по аксиоме Пересечения, так что $z={ }^{*} b \in \mathbb{S}$, и нетрудно проверить, что $X \cap y=z$ в $\mathfrak{E}$. Обратно, допустим, что $X \in \mathbf{E}$ удовлетворяет $\forall^{\text {st }} y \exists^{\text {st }} z(X \cap y=z)$ в $\mathfrak{E}$. Это означает, что для всякого множества $a \in \mathbf{V}$ существует $b \in \mathbf{V}, b \subseteq a$, такое, что $X \cap{ }^{*} a={ }^{*} b$ выполнено в $\mathfrak{E}$. Легко видеть, что такое $b=b_{a}$ единственно. Определим, в нашем базовом $\mathbf{G B}$-универсуме $\mathbf{K}$, класс $A=\bigcup_{a \in \mathbf{V}} b_{a}$. Тогда $Y={ }^{*} A \in \mathbf{S}$ и в $\mathfrak{E}$ истинно $Y \cap{ }^{*} a={ }^{*}\left(b_{a}\right)$ для любого $a$. Тем самым, в $\mathfrak{E}$ истинно $\forall^{\text {st }} y(X \cap y=Y \cap y)$, а тогда, согласно Ограниченности из $\mathbf{B S T}^{\text {sets }}, X=Y \in \mathbf{S}$.

Экстенсиональность в $\mathfrak{E}$ доказывается столь же простыми рассуждениями.

Стандартизация классов. Если $X \in \mathbf{E}$, то $A=\left\{b \in \mathbf{V}:{ }^{*} b \in X\right\}-$ класс в $\mathbf{K}$ (согласно Свертке теории $\mathbf{G B})$, и нетрудно проверить, что эквивалентность ${ }^{*} b{ }^{*} \in X \Leftrightarrow{ }^{*} b{ }^{*} \in{ }^{*} A$ выполнена для любого множества $b \in \mathbf{V}$. Теорема 2.16 доказана.

ЗАмЕчАНИЕ 2.20. Одним из возможных истолкований теоремы 2.16, в свете общей схемы ее доказательства, является то, что нестандартный универсум II множеств теории BST допускает расширение классами до универсума nGB (соответственно nKM) - в предположении, что класс $\mathbb{S}$ всех стандартных множеств допускает расширение классами до универсума GB (соответственно KM).

Этому результату можно придать точный смысл, введя теории классов в st$\in$-языке, постулирующие, что универсум всех множеств $\mathbb{I}$ удовлетворяет BST, каждый собственный класс $X$ удовлетворяет $X \subseteq \mathbb{S}$ и собственные классы, вместе со стандартными множествами, образуют универсум классов GB (соответственно KM). В такой теории мы можем определить стандартные, внутренние 
и внешние классы так, как указано выше в определении 2.17. Эта расширенная структура, в силу тех же рассуждений, будет интерпретацией nGB (или $\mathbf{n K M ) ~ в ~ т а к о и ̆ ~ п р о м е ж у т о ч н о и ̆ ~ т е о р и и . ~}$

Однако средств BST недостаточно для интерпретации $\mathbf{n G B}$, см. теорему 2.6.

2g. Метаматематические следствия. Главные метаматематические результаты, связывающие теории GB, nGB, KM, nKM между собой и с теориями множеств ZFC и BST, представлены на диаграммах 1 и 2 , где $A \longrightarrow B$ означает, что $A$ так или иначе сводится к $B$.

Диаграмма 1 представляет естественные сводимости "меньшей" теории к “большей". Вертикальные стрелки, помеченные st, обозначают интерпретацию посредством релятивизации к стандартным множествам или классам. Стрелки, помеченные sets, обозначают интерпретацию посредством релятивизации к подуниверсуму множеств (в универсуме классов). Стрелки, помеченные $\subset$, символизируют тот факт, что $\mathbf{G B}$ и $\mathbf{n G B}$ суть подтеории теорий $\mathbf{K M}$ и соответственно nKM.

Диаграмма 2 представляет менее тривиальные обратные сводимости. Перечеркнутые стрелки обозначают сводимости, не имеющие места по той причине, что непредикативные теории классов строго сильнее в смысле непротиворечивости, чем предикативные (см. конец п. 2а). Вертикальные стрелки, помеченные r, обозначают интерпретацию st-типа, в смысле замечания 1.18, нестандартных теорий в соответствующих стандартных структурах. Для пары ZFC, BST здесь речь идет о теореме 1.16 (см. обсуждение в замечании 1.18), а для двух пар теорий классов см. теорему 2.16 .

Наконец, стрелки, помеченные еq, cons (от equiconsistency, conservativity), означают, что сводимость теории классов к теории множеств имеет место по отношению к свойствам sets-консервативности и непротиворечивости, но не имеет места для интерпретируемости. Этот результат дается теоремами 2.2, 2.6.

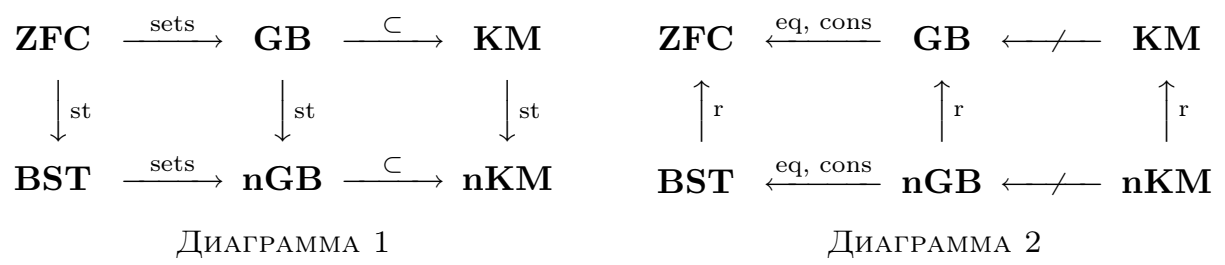

На каждой из двух диаграмм теории nGB и nKM могут быть заменены соответственно на $\mathbf{n G B}^{+}$и $\mathbf{n K M}^{+}$или на $\mathbf{n G B}+\sum_{2}^{\mathrm{st}}-$ классы и $\mathbf{n K M}+\Sigma_{2}^{\mathrm{st}}$-классы

Закончим этот пункт следующим результатом, усиливающим теорему 2.6.

СЛЕДСТВИЕ 2.21. Теории $\mathbf{n G B}+\Sigma_{2}^{\text {st }}$-классы $u \mathbf{n G B}^{+}-$все еще sets-консервативные и, следовательно, равнонепротиворечивые расширения BST.

ДокАЗАТЕЛЬство. Рассмотрим произвольную st- $\in$-формулу $\varphi$. Допустим (нетривиальное направление), что теория $\mathbf{n G B}+\Sigma_{2}^{\text {st }}$-классы доказывает $\varphi^{\text {sets }}$. Согласно следствию 1.21 , найдется $\in$-формула $\psi$ такая, что $\varphi \Leftrightarrow \psi \Leftrightarrow \psi^{\text {st }}$ выводится в BST, а тогда $\varphi^{\text {sets }} \Leftrightarrow \psi^{\text {sets }}$ выводится в nGB. Но $\psi^{\text {sets }}$ есть $\in$ формула, так что она выводима в $\mathbf{G B}$ по теореме 2.16 , а сама $\psi$ в ZFC по 
теореме 2.2. Наконец, $\psi$, а потому и данная формула $\varphi$ выводимы в BST по теореме 1.16(ii). Следствие 2.21 доказано.

Исторические и библиографические замечания к $§ 2$. Теория классов Гёделя-Бернайса GB восходит к работам этих авторов, а также фон Неймана в 1920-х-30-х годах. Название по книге [35]. В современной литературе также называется теорией фон Неймана-Бернайса-Гёделя NBG. Несколько позже стала известна ее связь с ZFC, выраженная в теореме 2.2. Об этом см. [53], [54], [36]. О GB-части теоремы 2.2 см., например, [35; гл. II, §6]. О непредикативной теории Келли-Морса КМ см. [55].

Нестандартная теория классов nGB была введена в [15] под названием NCT (nonstandard class theory) с несколько иным, чем у нас (но эквивалентным), списком аксиом. Первая формулировка нестандартной теории типа nKM дана в [20].

Главные результаты этого параграфа о редукции нестандартных теорий классов к стандартным, в частности, теорема 2.16 и ее метаматематические следствия, представленные в п. $2 \mathrm{~g}$, в части, относящейся к теориям ГёделяБернайса, получены П.В. Андреевым и Е.И. Гордоном [15], а в части, относящейся к теориям КМ-типа, К. Хрбачеком [20]. ${ }^{24}$ Ключевую роль в этих результатах играет $\Sigma_{2}^{\text {st }}$-определение внешних классов в п. $2 \mathrm{f}$ (ср. с теоремой 1.19). Хрбачек первый пришел к идее применить это определение не только для теорий GB-типа, где все достаточно просто, но и для теорий Келли-Морса, где такая возможность первоначально казалась маловероятной, поскольку предполагалось, что нестандартные теории КМ-типа должны быть сильнее в смысле непротиворечивости, чем "стандартная" KM, по аналогии с результатами из [42] для нестандартных систем арифметики высших порядков.

Термин “полумножество” был введен в рамках альтернативной теории множеств AST (см. [52], [51]). Понятие о стандартном размере появилось в работах по основаниям нестандартного анализа (впервые, вероятно, в [10]). Известно еще одно родственное понятие: класс $\mathbb{S}$-размера по Каваи [12], [13] - это такой класс $X$, для которого найдется класс $F$, являющийся отображением из $\mathbb{S}$ на $X$. Аксиома, названная здесь аксиомой $\Sigma_{2}^{\text {st }}$-классов, соответствует аксиоме хроматических классов в другом (в сущности, эквивалентном) варианте аксиоматизации предикативной нестандартной теории классов в [15].

ПроблемА 2.22. Можно ли добавить Перенос для произвольных формул к nGB без того, чтобы полученная теория стала равносильной KM в смысле непротиворечивости?

ПроБЛЕмА 2.23. По аналогии со сноской 13 назовем локалъно внутренним любой класс $X$ такой, что для любого множества $y$ класс $X \cap y$ является множеством. Ничего не известно о предложении $\mathbf{I}=\mathbf{I}^{\text {loc }}$ в нестандартных теориях классов: все локально внутренние классы являются внутренними.

\footnotetext{
${ }^{24}$ В целях унификации изложения исходные выкладки из [15], [20] здесь существенно модифицированы на основе схемы, ранее предложенной В.Г. Кановеем и М. Реекеном для теории BST в [21], [22], [25]. Технические детали этой модификации, в частности, теоремы 2.7 и 2.15, были найдены Кановеем после знакомства с препринтом Хрбачека [20] в августе 2004 г.
} 


\section{§ 3. Присоединение внешних множеств}

Этот параграф посвящен проблеме 3 из введения: как присоединить "внешние" множества к нестандартному универсуму "внутренних" множеств? Для начала уточним, что́ собственно здесь имеется в виду.

Напомним, что универсум таких нестандартных теорий, как BST или IST, или универсум множеств в теориях классов nGB, nKM удовлетворяет Переносу в форме $\Phi \Leftrightarrow \Phi^{\text {st }}$ ( $\Phi$ - любая $\in$-формула) и Выделению только в $\in$-языке, но не в расширенном st- $\in$-языке. Поэтому st- $\in$-определимая часть какого-то множества не обязательно будет множеством. (Лемма 1.14 дает простой пример.) Нестандартные теоретико-множественные универсумы этого типа, как и принадлежащие им множества, принято, по историческим причинам, называть "внутренними" (с кавычками или без). Другой тип универсумов, "внешних", характеризуется как раз обратными свойствами, т.е. Выделение выполнено в st$\in$-языке, но Перенос не имеет места. ${ }^{25}$ "Внешние" нестандартные универсумы обычно содержат подуниверсумы "внутреннего" типа: при подходящей аксиоматизации таковым будет класс всех внутренних множеств, т.е. тех, которые являются элементами стандартных множеств.

Здесь решается обратная задача: как расширить "внутренний" универсум, в этой терминологии, до “внешнего". Мы докажем (теорема 3.24), что универсум BST допускает расширение до универсума известной нестандартной теории множеств HST “внешнего" типа. (См. неформальный комментарий в конце п. 3е.) Более того (теорема 3.21), если данный универсум BST есть класс всех множеств в некоторой nGB- или $\mathbf{n K M - с т р у к т у р е , ~ т о ~ э т а ~ к л а с с о в а я ~ н а д с т р о и ̆ к а ~}$ продолжается до подходящей классовой надстройки над расширенным универсумом теории HST.

3а. Структура “внешнего” универсума теории Хрбачека. Желая расширить универсум BST до универсума нестандартной теории множеств c Выделением в st- $\in$-языке, мы должны прежде всего наметить контуры последнего. Начнем с аксиоматизации. Известно несколько разумных нестандартных теорий с Выделением в st- $\in$-языке. Наибольшее внимание из них привлекает теория множеств Хрбачека HST. Аксиомы теории HST разбиты на три группы.

Во-первых, это аксиомы Экстенсиональности, Пары, Объединения, Бесконечности в их обычных формах, принятых в ZFC, а также схемы Выделения и Собирания (поэтому также и Подстановки) для всех st- $\in$-формул. Аксиомы Степени, Выбора и Регулярности из ZFC не включаются: они противоречат HST, см. [25; раздел 1.3]. Но Регулярность и Выбор включены в ослабленных формах (Регулярность над $\mathbb{I}$, Выбор стандартного размера, Зависимый выбор, см. ниже), важных в контексте HST.

\footnotetext{
25 Другие варианты определения понятий “внешнее", "внутреннее” (множество или универсум), известные в основаниях нестандартного анализа, в сущности, равносильны данным в том смысле, что они выражают сходные идеи в тех областях, где применяются.
} 
Вторая группа содержит аксиомы для стандартных и внутренних множеств. Как обычно, индексы ${ }^{\text {st }}$ и ${ }^{\text {int }}$ означают релятивизацию к классам

$$
\begin{aligned}
& \mathbb{S}=\{x: \text { st } x\} \quad \text { (стандартные множества); } \\
& \mathbb{I}=\left\{x: \exists^{\mathrm{st}} y(x \in y)\right\} \quad \text { (внутренние множества). }
\end{aligned}
$$

$\mathbf{Z F C}^{\text {st }}$ : все формулы вида $\Phi^{\mathrm{st}}$, где $\Phi$ - аксиома $\mathbf{Z F C}$ в $\in$-языке.

Перенос: все формулы вида $\Phi^{\mathrm{st}} \Leftrightarrow \Phi^{\mathrm{int}}$, где $\Phi$ - замкнутая $\in$-формула со стандартными параметрами.

Транзитивность $\mathbb{I}: \forall x \in \mathbb{I} \forall y \in x(y \in \mathbb{I})$.

Регулярность над I: каждое непустое множество $X$ содержит элемент $x \in X$ такой, что $x \cap X \subseteq \mathbb{I}$. (Полная Регулярность теории ZFC требует $X \cap x=\varnothing$.)

Стандартизация: $\forall X \exists^{\mathrm{st}} Y(X \cap \mathbb{S}=Y \cap \mathbb{S})$.

Эти две группы аксиом организуют универсум $\mathbb{H}$ теории HST в виде ZFподобной (но без аксиомы Степени) надстройки над транзитивным классом $\mathbb{I} \subseteq \mathbb{H}$ всех внутренних множеств, который в свою очередь удовлетворяет BST.

В дополнение к методам BST, ограниченным областью II, теория HST допускает применение *-методов теоретико-модельного, "робинсоновского" нестандартного анализа. Именно, класс $\mathbb{W}$ всех фундированных множеств ${ }^{26}$ удовлетворяет ZFC и имеется st- $\in$-определимый $\in$-изоморфизм ${ }^{*}: \mathbb{W F} \stackrel{\text { на }}{\longrightarrow} \mathbb{S}$, являющийся, по Переносу, и элементарным вложением $\langle\mathbb{W} \mathbb{F} ; \in\rangle$ в $\langle\mathbb{I} ; \in\rangle$. Именно, ${ }^{*} x \in \mathbb{S}$ определяется для каждого $x \in \mathbb{W} \mathbb{F}$ при помощи $\in$-индукции так, что * $x$ есть то единственное стандартное множество, для которого ${ }^{*} x \cap \mathbb{S}=\left\{{ }^{*} y: y \in x\right\}$. См. об этом [25; гл. 1].

Наконец, третья группа включает следующие вариации аксиом из п. 2е, относящиеся к множествам стандартного размера. Среди них Насыщенность является основой типично "нестандартных" рассуждений, а обе аксиомы выбора также играют важную роль в некоторых построениях.

Выбор стандартного размера: если $X$ - множество стандартного размера, а $P$ - любое множество, то существует функция $f$ такая, что $X \subseteq \operatorname{dom} f$ и, для любого $x \in X, \exists y(\langle x, y\rangle \in P) \Rightarrow\langle x, f(x)\rangle \in P$.

Насыщенность: если $H \subseteq \mathbb{I}$ - множество стандартного размера, не содержащее $\varnothing$ и $\cap$-замкнутое, то пересечение $\bigcap H$ непусто.

Зависимый выбор: для любых множеств $X$ и $R \subseteq X \times X$ с $\operatorname{dom} R=X$ найдется последовательность $\left\{x_{n}\right\}_{n \in \mathbb{N}}$ такая, что $\left\langle x_{n}, x_{n+1}\right\rangle \in R$ для всех $n \in \mathbb{N}$.

Аналогично определению 2.13, множеством стандартного размера называется в HST любое множество $X$, для которого найдутся стандартное множество $x$ и биекция $F$ (также множество) из $x \cap \mathbb{S}$ на $X$. Заметим, что в $\mathbf{H S T}$ все полумножества являются множествами, поскольку Выделение принято для всех st- $\in$-формул.

В формулировке Зависимого выбора $\mathbb{N}$ обозначает натуральные числа. Простые рассуждения показывают, что $\mathbb{N}=(\mathbb{N})^{\mathbb{W}}$, т.е. натуральные числа можно с тем же успехом определить в ZFC-универсуме $\mathbb{W F}$ всех фундированных множеств. В то же время имеется и большее множество * $\mathbb{N} \in \mathbb{I}$ всех $\mathbb{I}$-натуральных,

\footnotetext{
${ }^{26}$ Множество $x$ фундировано (well-founded, откуда аббревиатура $\mathbb{W} \mathbb{F}$ ), когда найдется транзитивное множество $T$ такое, что $x \subseteq T$ и отношение $\in \uparrow T$ фундировано, т.е. любое непустое $Y \subseteq T$ содержит $\in$-наименьший элемент.
} 
или гипернатуральных, чисел, причем $\mathbb{N}={ }^{*} \mathbb{N} \cap \mathbb{S}={ }^{*} \mathbb{N} \cap \mathbb{W} \mathbb{F}-$ начальный сегмент ${ }^{*} \mathbb{N}$. В целом же пара множеств $\mathbb{N},{ }^{*} \mathbb{N}$ в $\mathbf{H S T}$ соответствует паре $\mathbb{N} \cap \mathbb{S}, \mathbb{N}$ в BST.

Обозначим $\mathbf{H S T}^{+}$теорию, полученную присоединением к $\mathbf{H S T}$ следующих двух аксиом, преимущественно технического характера. Об их роли будет сказано ниже. Напомним, что $E_{p}$ и $\mathbf{C}_{0}$ определены в п. 2 e.

$\Sigma_{2}^{\text {st }}$-множества: для любого множества $x \subseteq \mathbb{I}$ есть такой код $p \in \mathbf{C}_{0}$, что $x=E_{p}$.

$\mathbb{I}$-образ: для любого множества $d$ существуют множество $r \in \mathbb{I}$ и функция $f$ (тоже множество), определенная на $r$, для которых $d \subseteq \operatorname{ran} f$.

В плане метаматематических вопросов, теории $\mathbf{H S T}$ и $\mathbf{H S T}^{+}$относятся к BST примерно так же, как последняя относится к ZFC в рамках теоремы 1.16, в частности, HST и $\mathbf{H S T}^{+}$равнонепротиворечивы с BST (теорема 3.24 ниже). Эта редукция $\mathbf{H S T}$ и $\mathbf{H S T}^{+}$к $\mathbf{B S T}$ довольно сложна. Обратное же направление не доставляет затруднений; простой вывод следующего результата оставлен читателю.

ПРЕДЛОЖЕНИЕ 3.1. Структура $\langle\mathbb{I} ; \in, \mathrm{st}\rangle$ есть интерпретация BST в HST. Другими словами, в HST выводится $\varphi^{\text {int }}$ для всякой аксиомы $\varphi$ теории BST.

3b. Нестандартные теории классов на базе теории Хрбачека. Нестандартные теории из следующего определения характеризуют свои универсумы как классовые надстройки (по типу Гёделя-Бернайса или по типу КеллиMopca) над универсумом множеств теории $\mathbf{H S T}$ или $\mathbf{H S T}^{+}$, в точности аналогично тому, как универсумы теорий nGB и nKM являются классовыми надстройками над универсумом BST.

ОПРЕДЕЛЕНИЕ 3.2. GB/HST (т.е. GB-расширение HST) есть теория классов в st- $\in$-языке, которая содержит схему $\mathbf{H S T S}^{\text {sets }}$, т.е. все аксиомы HST, формально релятивизованные к классу всех множеств $\{x: \operatorname{set} x\}$, и, как в определении 2.3, Пересечение, Экстенсиональность, Собирание, схему Свертки для предикативных st- $\in$-формул, Стандартизацию классов, а также аксиому $\mathbf{S}=\mathbf{S}^{\text {loc }}$ для классов $X \subseteq \mathbb{I} .^{27}$

Теория KM/HST (т.е. KM-расширение HST) определяется аналогично с добавлением пунктов 1) и 2) определения 2.3 .

Аналогичным образом определяются и теории GB/HST ${ }^{+}$и $\mathbf{K M} / \mathbf{H S T}^{+}$: только вместо $\mathbf{H S T S}^{\text {sets }}$ берем схему $\left(\mathbf{H S T}^{+}\right)^{\text {sets }}$. Это, очевидно, то же самое, что просто присоединить аксиомы $\mathbb{I}$-образа и $\Sigma_{2}^{\text {st }}$-множеств к теориям соответственно GB/HST и KM/HST.

Примем, для работы в этих теориях, обозначения областей из определения 2.4; но равенства $\mathbb{I}=\mathbb{H}$ и $\mathbf{E}=\mathbf{H}$ здесь уже не имеют места. Добавим, что в теории HST выполнено $\mathbb{S}=\mathbf{S} \varsubsetneqq \mathbb{I}=\mathbf{I} \varsubsetneqq \mathbb{H}=\mathbf{E}=\mathbf{H}$. Следующий простой результат (для экономии места доказательство опущено) демонстрирует характер взаимоотношений между универсумами теорий из определения 3.2

\footnotetext{
${ }^{27}$ Аксиома $\mathbf{S}=\mathbf{S}^{\text {loc }}$ не верна даже для множеств в $\mathbf{H S T}$, поскольку существуют непустые множества, вообще не пересекающие $\mathbb{I}$, а любой стандартный класс $Y$ удовлетворяет $Y \subseteq \mathbb{I}$.
} 
и теорий nGB, nKM. Напомним, что $\mathbf{E}$ обозначает область всех классов $X \subseteq \mathbb{I}$, т.е. классов внутренних множеств.

ПРЕДЛОЖЕНИЕ 3.3. Структура $\mathfrak{E}=\langle\mathbf{E} ; \in$, st $\rangle$ есть интерпретация nGB в GB/HST, m.е. в GB/HST въводится $\varphi^{\mathfrak{E}}$ для всякой аксиомы $\varphi$ теории nGB. ${ }^{28}$

Кроме того, $\mathfrak{E}$ является интерпретацией теории $\mathbf{n G B}^{+}{ }_{\text {в }} \mathbf{G B} / \mathbf{H S T}^{+} u$ интерпретацией теории $\mathbf{n K M}^{+}$в $\mathbf{K M} / \mathbf{H S T}^{+}$.

Наш анализ метаматематических свойств теорий из определения 3.2 фокусируется на $\mathbf{G B} / \mathbf{H S T}^{+}$и $\mathbf{K M} / \mathbf{H S T}^{+}$, а не на паре $\mathbf{G B} / \mathbf{H S T}$ и $\mathbf{K M} / \mathbf{H S T}$, которая на первый взгляд выглядит более естественной. Здесь дело в следующем.

При расширении универсума, скажем, теории nGB до универсума GB/HST, все полумножества $X \subseteq \mathbb{I}$ с необходимостью становятся множествами, а потому, чтобы обеспечить Свертку в расширенной области, мы должны иметь эту схему в каком-то виде для полумножеств уже в $\mathbf{n G B}$. Однако полумножества - это технически классы в $\mathbf{n G B}$, так что никакого прямого метода ввести для них Свертку, пожалуй, нет. Косвенный же метод состоит в том, чтобы постулировать параметризацию всех полумножеств $X \subseteq \mathbb{I}$ собственно множествами из $\mathbb{I}$. В присутствии аксиомы $\Sigma_{2}^{\text {st }}$-полумножеств такая параметризация дается в $\mathbf{n G B}$ отображением $p \mapsto E_{p}$.

Это заставляет нас присоединить аксиому $\Sigma_{2}^{\text {st }}$-полумножеств к $\mathbf{n G B}$, получая теорию $\mathbf{n G B}^{+}$и, соответственно, теорию GB/HST с аксиомой $\Sigma_{2}^{\text {st }}$-полумножеств согласно предложению 3.3. Но в GB/HST все полумножества $X \subseteq \mathbb{I}$ являются множествами, так что аксиома $\Sigma_{2}^{\text {st }}$-множеств присоединяется к HST. Разумеется, существуют и другие подходящие параметризации, но та, которая дается отображением $p \mapsto E_{p}$ и аксиомой $\Sigma_{2}^{\text {st }}$-полумножеств, несомненно самая удобная.

Присоединение второй дополнительной аксиомы II-образа также вызвано определенной причиной. Она связана с аксиомой Собирания Классов теории $\mathbf{n K M}$ в предложении 3.3. Дело в том, что Собирание Классов в $\mathfrak{E}$ не удается прямо вывести из Собирания Классов в универсуме теории KM/HST: поскольку то множество $r$, которое дается Собиранием Классов в универсуме, может не иметь ничего общего с областью II. Однако аксиома I-образа немедленно исправляет ситуацию.

Обосновав таким образом необходимость присоединения аксиом $\mathbb{I}$-образа и $\Sigma_{2}^{\text {st }}$-полумножеств (или $\Sigma_{2}^{\text {st }}$-множеств для теории $\mathbf{H S T}^{+}$), займемся метаматематическими связями нестандартных теорий классов $\mathbf{G B} / \mathbf{H S T}^{+}$и $\mathbf{K M} / \mathbf{H S T}^{+}$ с нестандартной теорией множеств $\mathbf{H S T}^{+}$, с одной стороны, и с теориями классов $\mathbf{n G B}^{+}$и $\mathbf{n} \mathbf{K M}^{+}-$с другой стороны. Первый аспект раскрывается следующим образом.

Tеорема 3.4. Теория GB/HST ${ }^{+}$есть sets-консервативное расширение теории $\mathrm{HST}^{+}$в том же смысле, что в теореме 2.6 (для всех st- $\epsilon$-формул $\varphi$ ), а потому GB/HST ${ }^{+}$равнонепротиворечива с $\mathbf{H S T}^{+}, \mathbf{H S T}, \mathbf{B S T}, \mathbf{Z F C}$.

\footnotetext{
${ }^{28}$ Следует отметить, что любое $\mathbf{G B} / \mathbf{H S T}$-множество $x \in \mathbb{H} \backslash \mathbb{I}, x \subseteq \mathbb{I}$, становится собственным классом, точнее, полумножеством в $\mathfrak{E}$.
} 
Однако GB/HST ${ }^{+}$не допускает интерпретации в $\mathbf{H S T}^{+}$, a $\mathbf{K M} / \mathbf{H S T}^{+}$ даже не равнонепротиворечива с $\mathbf{H S T}^{+}$.

ДокАЗАТЕЛЬСтво. Понятно, что структура $\langle\mathbb{H} ; \in, \mathrm{st}\rangle$ есть интерпретация $\mathbf{H S T}^{+}{ }_{\text {в }} \mathbf{G B} / \mathbf{H S T}^{+}$. С другой стороны, совершенно аналогично доказательству теоремы 2.6, каждая модель $\mathbf{H S T}^{+}$может быть расширена, присоединением классов, до модели GB/HST ${ }^{+}$(где данная модель $\mathbf{H S T}^{+}$остается классом всех множеств). Отсюда следует sets-консервативность и равнонепротиворечивость с $\mathbf{H S T}{ }^{+}$. Для доказательства остальных утверждений достаточно принять на веру, что $\mathbf{H S T}^{+}$допускает интерпретацию в BST (см. ниже). Ис-

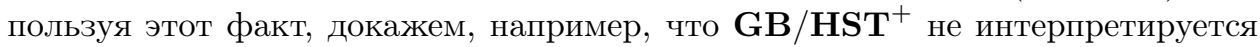
в $\mathbf{H S T}^{+}$. В самом деле, иначе $\mathbf{G B} / \mathbf{H S T}^{+}$интерпретируется и в BST (по предположению), а тогда и nGB интерпретируется в BST (согласно предложению 3.3), противоречие с теоремой 2.6. Теорема 3.4 доказана.

В плане взаимоотношений с $\mathbf{n G B}$ и nKM, результат в одну сторону дается предложением 3.3, но редукция в другую сторону потребует значительно больше работы.

3c. Кумулятивное построение множеств и классов. B этом пункте мъ рассуждаем в $\mathbf{n G B}$. Дополнительные аксиомы из $\mathbf{n G B}^{+}$и $\mathbf{n K M} \mathbf{M}^{+}$будут вводиться по мере надобности и явно указываться.

Наша главная цель - это расширение всего универсума I теории nGB до теоретико-классового универсума $\mathbf{H}$, который, в определенных дополнительных предположениях, будет удовлетворять аксиомам GB/HST ${ }^{+}$или $\mathbf{K M} / \mathbf{H S T}^{+}$.

Одновременно класс $\mathbb{I}=\{x: \operatorname{set} x\}$ всех множеств в $\mathbf{n G B}$ будет расширен до некоторого теоретико-множественного универсума $\mathbb{H}$, удовлетворяющего аксиомам HST (или, при некоторых дополнительных предположениях, $\mathbf{H S T}^{+}$) и являющегося частью H. В соответствии с общей структурой универсума $\mathbf{H S T}$, описанной в п. За, II образует самый нижний уровень $\in$-иерархии $\mathbb{H}$. Следующий уровень будет состоять из полумножеств. Затем, для продолжения построения универсума HST на базе II, мы должны образовать совокупности полумножеств, вроде $\left\{X_{p}: p \in Y\right\}$, где $X, Y$ - полумножества и $X_{p}=\{x:\langle p, x\rangle \in X\}$, и т. д. Разумеется, нет никакой надежды осуществить это построение в прямой форме в nGB, где собственные полумножества ничему не могут принадлежать, так что уже уровень сразу над полумножествами никак не может быть построен в явном виде. Однако такое построение можно осуществить в закодированной форме.

Мы используем известный метод кодировки кумулятивного построения множеств при помощи фундированных деревьев, который в общем виде выглядит так. Допустим, что $T$ - фундированное (т.е. не имеющее бесконечных ветвей) дерево, в котором каждой вершине (максимальному элементу) сопоставлено множество $f(t)$. В этом случае можно определить $A_{T f}(t)$ для каждого $t \in T$ так, что $A_{T f}(t)=f(t)$ для всех вершин $t$ и $A_{T f}(t)=\left\{A_{T}(s)\right.$ : $s$ непосредственно следует за $t\}$ для $t$, не являющихся вершинами. Положим $A_{T f}=A_{T f}(\Lambda)$, где $\Lambda$ - корневая точка $T$.

Теперь посмотрим, как этот метод выглядит с учетом особенностей nGB. 
ОБЩЕЕ СОГЛАШЕНИЕ 3.5. В частичное изменение соглашений п. 2а об употреблении букв, строчные буквы (в том числе полужирные), в зависимости от контекста, могут обозначать также и полумножества, не являющиеся множествами.

Если не оговорено противное, то “последовательность" будет обозначать "множество, являющееся последовательностью", а слова "функция", “дерево", напротив, будут типично обозначать "класс, являющийся функцией, деревом".

\section{А. О конечности и полной упорядоченности в связи с классами.} Это техническое отступление, необходимое для обоснования некоторых нижеследующих построений. Напомним, что вполне упорядоченным называется всякое линейно упорядоченное множество, каждое непустое подмножество которого имеет наименьший элемент. Например, в ZFC каждый ординал вполне упорядочен отношением $\in$, а натуральный ряд $\mathbb{N}=\omega$ вполне упорядочен своим естественным порядком (который формально совпадает с $\in$ ). Это верно и в теории BST (поскольку все названные факты выражаются $\in$-формулами, a BST влечет ZFC в $\in$-языке) и тем самым верно и в $\mathbf{n G B}$ (так как эта теория является sets-консервативным расширением BST).

Однако в теории nGB появляется новый аспект: классы, даже подклассы множеств (т.е. полумножества), не являющиеся множествами. Например, $\mathbb{N}$ имеет непустое подполумножество $\mathbb{N} \backslash \mathbb{S}$ (нестандартных натуральных чисел) без наименьшего элемента (см. лемму 1.14). Это приводит к следующему определению: линейно упорядоченное множество или класс является $\kappa$-вполне упорядоченным (префикс "к" от “класс"), если всякий его непустой подкласс имеет наименьший элемент.

ПрЕДЛОЖЕНИЕ 3.6 (nGB).

(i) Класс Ord $\cap \mathbb{S}$ всех стандартных ординалов и тем самым полумножество $\mathbb{N} \cap \mathbb{S}$ являются к-вполне упорядоченными.

(ii) Для всякого полумножества $X \subseteq$ Ord найдется наименъший стандартный ординал, строго больший, чем все ординаль из $X$.

(iii) Любая последовательность-полумножество $\left\langle x_{1}, \ldots, x_{n}\right\rangle$ длины $n \in \mathbb{N} \cap \mathbb{S}$, все члены $x_{i}$ которой - множества, является множеством.

ДокАЗАтельство. (i) Докажем, что любой непустой класс $X \subseteq \operatorname{Ord} \cap \mathbb{S}$ имеет наименьший элемент. Согласно Стандартизации, найдется стандартный класс $Y$ такой, что $Y \cap \mathbb{S}=X \cap \mathbb{S}=X$. Наименьший ординал $\xi$, содержащийся в $Y$, стандартен по Переносу. Поэтому $\xi$ - наименьший элемент в $X$.

Результат для $\mathbb{N} \cap \mathbb{S}$ является обоснованием индукции по стандартным натуральным числам в случае, когда индуктивный переход описывается st- $\in$-формулой, например, ссылается на полумножества. В частности, для вывода (iii) заметим, что по определению $\left\langle x_{1}, \ldots, x_{n}, x_{n+1}\right\rangle=\left\langle\left\langle x_{1}, \ldots, x_{n}\right\rangle, x_{n+1}\right\rangle$, так что если $\left\langle x_{1}, \ldots, x_{n}\right\rangle$ и $x_{n+1}-$ множества, то и $\left\langle x_{1}, \ldots, x_{n}, x_{n+1}\right\rangle$ - множество. Предложение 3.6 доказано.

Б. Деревья. Обозначим Seq класс всех последовательностей $\left\langle a_{1}, \ldots, a_{n}\right\rangle$ (произвольных множеств $a_{i} \in \mathbb{I}$ ) стандартной конечной длины. Для $t \in \mathrm{Seq}$ и любого множества $a t^{\wedge} a$ есть последовательность в Seq, полученная присоединением $a$ справа к $t$. Обозначение $a^{\wedge} t$ понимается аналогично. Запись 
$t^{\prime} \subseteq t$ означает, что последовательность $t \in \mathrm{Seq}$ продолжает $t^{\prime} \in \mathrm{Seq}$ (возможно, $t^{\prime}=t$ ), а $t^{\prime} \subset t$ означает, что $t$ - собственное продолжение $t^{\prime}$ (так что $t^{\prime} \neq t$ ). Через $\langle a\rangle$ обозначается последовательность с единственным членом $a$, а $\Lambda$ пустая последовательность.

- Деревом называется любой непустой класс $T \subseteq$ Seq такой, что для любой пары последовательностей $t^{\prime}, t \in$ Seq с $t^{\prime} \subseteq t$ мы имеем $t \in T \Rightarrow t^{\prime} \in T$.

- Мах $T$ обозначает класс всех $\subseteq$-максимальных элементов $r \in T$.

- Если $t \in T$, то положим $\operatorname{Succ}_{T}(t)=\left\{a: t^{\wedge} a \in T\right\}$ (это также класс).

- Определим $\operatorname{Min} T=\operatorname{Succ}_{T}(\Lambda)=\{a:\langle a\rangle \in T\} ;$ тогда $\operatorname{Min} T=\varnothing \Leftrightarrow$ $T=\{\Lambda\}$.

- Скажем, что $s \in T$ - элемент 1-го рода в $T$, если класс $T_{s}=\{t \in T$ : $s \subseteq t\}$ является полумножеством (как частный случай, множеством), и элемент 2-го рода в противном случае (т.е. когда класс $T_{s}$ не накрывается множеством).

Скажем, что дерево $T$ к-фундировано, когда каждый непустой класс $T^{\prime} \subseteq T$ содержит элемент, $\subseteq$-максимальный в $T^{\prime}$. В этом случае, следуя примерам из ZFC, мы хотели бы определить функцию ранга на $T$. Учитывая предложение 3.6, эта функция должна принимать значения в $\operatorname{Ord} \cap \mathbb{S}$, а не в Ord. Kроме того, поскольку $T$ может быть "большим" классом (не полумножеством), следует зарезервировать символ $\infty$, который считается бо́льшим, чем все стандартные ординалы.

Для всякого класса $X \subseteq(\operatorname{Ord} \cap \mathbb{S}) \cup\{\infty\}$ определим $\sup ^{\mathbb{S}} X=\infty$, когда $\infty \in X$ или же $X \subseteq \operatorname{Ord} \cap \mathbb{S}$ неограничено в $\operatorname{Ord} \cap \mathbb{S}$, а если $X \subseteq \operatorname{Ord} \cap \mathbb{S}$ ограничено в $\operatorname{Ord} \cap \mathbb{S}$, т.е. является полумножеством, то $\sup ^{\mathbb{S}} X$ равно наименьшему стандартному ординалу, строго превосходящему все ординалы из $X$. (Мы ссылаемся на предложение 3.6.)

Лемма 3.7. (nKM) Если $T \subseteq$ Seq - фундированное дерево, то существует единственное отображение $t \mapsto|t|_{T}^{\mathbb{S}}$ из $T$ в $(\operatorname{Ord} \cap \mathbb{S}) \cup\{\infty\}$ такое, что $|t|_{T}^{\mathbb{S}}=$ $\sup _{t^{\wedge} a \in T}^{\mathbb{S}}\left|t^{\wedge} a\right|_{T}^{\mathbb{S}}$ для каждого $t \in T$.

(nGB+пм-ограниченная Свертка) То же самое верно в предположении, что все $t \in T$, кроме, возможно, $\Lambda$, являются элементами 1-го рода в дереве $T$.

Отображение $t \mapsto|t|_{T}^{\mathbb{S}}$ является функиией ранга на (к-фундированном) дереве $T$. Понятно, что $|t|_{T}^{\mathbb{S}}=0$ при $t \in \operatorname{Max} T$. Положим $|T|_{T}^{\mathbb{S}}=|\Lambda|_{T}^{\mathbb{S}}($ высота $T)$.

ДокАЗАтельство. ${ }^{29}$ Рассмотрим подробнее случай nKM. Докажем, что для всякого $s \in T$ существует единственный класс $F_{s}$, являющийся функцией из $T_{s}=\{t \in T: s \subseteq t\}$ в $(\operatorname{Ord} \cap \mathbb{S}) \cup\{\infty\}$ и удовлетворяющий $F_{s}(t)=$ $\sup _{t^{\wedge} a \in T} F_{s}\left(t^{\wedge} a\right)$ для всех $t \in T_{s}$. Класс $S$ всех $s \in T$, для которых это утверждение существования и единственности $F_{s}$ имеет место, индуктивен в $T$, в том смысле, что мы имеем $s \in S$ всякий раз, когда $s^{\wedge} a \in S$ выполнено для любого $s^{\wedge} a \in T-$ в частности, каждое $s \in \operatorname{Max} T$ принадлежит $S$. (В самом деле, определим $F_{s}(t)=F_{s^{\wedge} a}(t)$ при $s^{\wedge} a \subseteq t$, и дополнительно $F_{s}(s)=\sup _{s^{\wedge} a \in T}^{\mathbb{S}} F_{s^{\wedge} a}\left(s^{\wedge} a\right)$.) Однако, из-за к-фундированности $T$, любой индуктивный класс $S \subseteq T$ совпадает с $T$. (Иначе получаем противоречие, рассматривая максимальный элемент непустой разности $T \backslash S$.)

\footnotetext{
${ }^{29}$ Метод доказательства называется фундированной индукцией.
} 
В случае, когда предполагается только nGB с пм-ограниченная Сверткой, рассуждение, вообще говоря, не проходит, поскольку определение $F_{s}$ при условии, что известны все $F_{s^{\wedge}} a$, апеллирует к Непредикативной Свертке. Все же если $s \in T$ - элемент 1-го рода, т.е. $T_{s}=\operatorname{dom} F_{s}-$ полумножество, то $F_{s}-$ также полумножество по Собиранию. Поэтому в наших условиях класс $S$ может быть определен пм-ограниченной формулой (т.е. все кванторы ограничены полумножествами, см. определение 2.13), так что Непредикативную Свертку можно заменить пм-ограниченной Сверткой. Лемма 3.7 доказана.

В. Коды. Следующее определение несколько искусственно заменяет пару $\langle T, f\rangle$, как выше в начале п. 3с, одной функцией $f$, обозначаемой через $\mathbf{X}$. Причина в том, что пары полумножеств не существуют в nGB. C другой стороны, к-фундированное дерево восстанавливается из своих максимальных элементов.

ОПРЕДЕЛЕНИЕ 3.8 (nGB). A-кодом (от: assembling) называется любая функция (класс) $\mathbf{X}: D \rightarrow \mathbb{I}$, определенная на каком-нибудь классе $D \subseteq$ Seq, состоящем из попарно $\subseteq$-несравнимых последовательностей и таком, что $T[\mathbf{X}]=\{t \in$ Seq : $\left.\exists t^{\prime} \in \operatorname{dom} \mathbf{x}\left(t \subseteq t^{\prime}\right)\right\}$ есть к-фундированное дерево. (Тогда $\operatorname{Max} T[\mathbf{X}]=$ $D=\operatorname{dom} \mathbf{x}$.)

А-код $\mathbf{X}$ называется регулярным, если $\left\{\mathbf{X}\left(t^{\wedge} a\right): t^{\wedge} a \in T[\mathbf{X}]\right\} \notin \mathbb{I}$ (т.е. этот класс не является множеством) всякий раз, когда $t \in T[\mathbf{X}]$ удовлетворяет $|t|_{T}^{\mathbb{S}}=$ 1 (т.е. $t \notin \operatorname{Max} T[\mathbf{X}]$, но любое $t^{\wedge} a \in T[\mathbf{X}]$ принадлежит $\operatorname{Max} T[\mathbf{X}]$ ).

Совокупность всех регулярных кодов $\mathbf{X}$ таких, что каждый элемент $s \in$ $T[\mathbf{X}]$, кроме, возможно, $\Lambda$, является элементом 1-го рода в $T[\mathbf{X}]$ (т.е. $\left\{a: s^{\wedge} a \in\right.$ $\mathbf{X}\}$ - полумножество), обозначается $\mathbf{H}$. Совокупность всех кодов $\mathbf{X} \in \mathbf{H}$ таких, что дерево $T[\mathbf{X}]$ (а тогда и само $\mathbf{X}$ ) есть полумножество, обозначается $\mathbf{h}$, а коды из $\mathbf{h}$ будут типично обозначаться строчными полужирными буквами $\mathbf{x}, \mathbf{y}, \ldots$

Требование регулярности не умаляет общности, ибо если $|t|_{T}^{\mathbb{S}}=1$ и $y=$ $\left\{\mathbf{X}\left(t^{\wedge} a\right): t^{\wedge} a \in T[\mathbf{X}]\right\} \in \mathbb{I}$ (т.е. $y-$ множество), то можно взять редуцированный код $\mathbf{Y}$ такой, что $T[\mathbf{Y}]=T[\mathbf{X}] \backslash\left\{t^{\wedge} a: t^{\wedge} a \in T[\mathbf{X}]\right\}$ и $\mathbf{Y}(t)=y$.

Интерпретация кумулятивной кодировки основана на следующем определении:

ОПРЕДЕЛЕНИЕ 3.9 (nGB). Пусть $\mathbf{X} \in \mathbf{H}$. Положим $\mathbf{F}_{\mathbf{X}}(t)=\mathbf{X}(t)$ при $t \in$ $\operatorname{Max} T[\mathbf{X}]$ и $\mathrm{F}_{\mathbf{X}}(t)=\left\{\mathrm{F}_{\mathbf{X}}\left(t^{\wedge} a\right): t^{\wedge} a \in T[\mathbf{X}]\right\}$ при $t \notin \operatorname{Max} T[\mathbf{X}]$.

Положим $\mathrm{A}_{\mathbf{X}}=\mathrm{F}_{\mathbf{X}}(\Lambda)$ (класс, кодируемый кодом $\mathbf{X}$ ).

Лемма 3.10 (nGB). Пусть $\mathbf{X} \in \mathbf{H} u t \in T[\mathbf{X}]$. Тогда $\mathbf{F}_{\mathbf{X}}(t)$ - множсество nри $|t|_{T[\mathbf{X}]}^{\mathbb{S}}=0$, собственный класс при $|t|_{T[\mathbf{X}]}^{\mathbb{S}} \leqslant 1$ и не определено (по определению 3.9) при $|t|_{T[\mathbf{X}]}^{\mathbb{S}} \geqslant 2$. В частности, $\mathrm{A}_{\mathbf{X}}$ определено при $|T[\mathbf{x}]|^{\mathbb{S}} \leqslant 1$ и не определено иначе.

ДокАЗАтельство. Значения $\mathbf{F}_{\mathbf{X}}(t)=\mathbf{X}(t) \in \mathbb{I}$ прямо определены при $t \in$ $\operatorname{Max} T[\mathbf{X}]$. Если $t \in T[\mathbf{X}]$ и $|t|_{T[\mathbf{X}]}^{\mathbb{S}}=1$, то все $t^{\wedge} a \in T[\mathbf{X}]$ принадлежат $\operatorname{Max} T[\mathbf{X}]$, а потому $\mathrm{F}_{\mathbf{X}}(t)=\left\{\mathrm{F}_{\mathbf{X}}\left(t^{\wedge} a\right): t^{\wedge} a \in T[\mathbf{X}]\right\}$ - корректно определенный класс, причем не множество согласно регулярности кода $\mathbf{X}$. При $|t|_{T[\mathbf{X}]}^{\mathbb{S}}=2$ определение 3.9 дает совокупность, состоящую из собственных классов, что невозможно в nGB. Лемма 3.10 доказана.

Случай $|T[\mathbf{x}]|^{\mathbb{S}} \leqslant 1$ рассматривается в пунктах 1) и 2) следующего примера. 
Пример 3.11. 1) Для любого множества $x \in \mathbb{I}$ определим А-код ${ }^{\mathrm{a}} x \in \mathbf{h}$ так, что $T\left[{ }^{\mathrm{a}} x\right]=\{\Lambda\}$ и ${ }^{\mathrm{a}} x(\Lambda)=x$, т.е. формально ${ }^{\mathrm{a}} x=\{\langle\Lambda, x\rangle\}$. В этом случае функция $\mathrm{F}_{\mathrm{a}_{x}}(\cdot)$, очевидно, существует и $x=\mathrm{A}_{\mathrm{a}_{x}}$.

2) Если $X$ - класс, не являющийся множеством, то определение ${ }^{\text {a }} X$ согласно 1) не работает, поскольку собственные классы не могут быть элементами классов. Определим в этом случае А-код ${ }^{\mathrm{a}} X$ так, что $T\left[{ }^{\mathrm{a}} X\right]=\{\Lambda\} \cup\{\langle a\rangle: a \in X\}$ и ${ }^{\mathrm{a}} X(\langle a\rangle)=a$ для каждого $a \in X$. Таким образом, $\operatorname{Max} T\left[{ }^{\mathrm{a}} X\right]=\{\langle a\rangle: a \in X\}$, $\operatorname{Min} T\left[{ }^{\mathrm{a}} X\right]=\{a: a \in X\}=X$. Регулярность ${ }^{\mathrm{a}} X$ следует из предположения, что $X$ - не множество. Более того, ${ }^{\text {a }} X \in \mathbf{H}$, а если $X-$ полумножество, то ${ }^{\mathrm{a}} X \in \mathbf{h}$. Функция (класс) $\mathrm{F}_{\mathrm{a} X}(\cdot)$ существует для любого класса $X$ и $X=\mathrm{A}_{\mathrm{a} X}$.

ОПРЕДЕЛЕНИЕ 3.12. Пусть $\mathbf{X} \in \mathbf{H}$ и $b \in \operatorname{Min} T[\mathbf{X}]$. Определим код $\mathbf{Y}=\left.\mathbf{X}\right|_{b}$ так, что $T\left[\left.\mathbf{X}\right|_{b}\right]=\left\{t: b^{\wedge} t \in T[\mathbf{X}]\right\}$ и $\left.\mathbf{X}\right|_{b}(t)=\mathbf{X}\left(b^{\wedge} t\right)$ для каждого $t \in \operatorname{Max} T[\mathbf{X}]$. Легко видеть, что $\left.\mathbf{X}\right|_{b} \in \mathbf{H}$, даже $\in \mathbf{h}$, и $\operatorname{Max} T\left[\left.\mathbf{X}\right|_{b}\right]=\left\{t: b^{\wedge} t \in \operatorname{Max} T[\mathbf{X}]\right\}$.

Г. Бисимуляции. Несмотря на ограничения, даваемые леммой 3.10, имеется метод, позволяющий адекватно определить, в nGB, отношения равенства и принадлежности между $A_{\mathbf{X}}$ и $A_{\mathbf{Y}}(\mathbf{X}, \mathbf{Y} \in \mathbf{H})$ даже в случае, когда множества или классы $A_{\mathbf{X}}, A_{\mathbf{Y}}$ не определены. Метод основан на следующем определении.

ОПРЕДЕЛЕНИЕ 3.13. Функция (класс) $j: T[\mathbf{X}] \times T[\mathbf{Y}] \rightarrow\{0,1\}$ называется бисимуляцией для А-кодов $\mathbf{X}, \mathbf{Y}$, если выполнены следующие условия.

$1^{*}$. Если $t \in \operatorname{Max} T[\mathbf{X}]$ и $r \in \operatorname{Max} T[\mathbf{Y}]$, то $j(t, r)=1$ равносильно $\mathbf{X}(t)=$ $\mathbf{Y}(r)$.

$2^{*}$. Если $t \in \operatorname{Max} T[\mathbf{X}]$, но $r \notin \operatorname{Max} T[\mathbf{Y}]$, или наоборот, $t \notin \operatorname{Max} T[\mathbf{X}]$, но $r \in \operatorname{Max} T[\mathbf{Y}]$, то $j(t, r)=0$. (Без предположения регулярности кодов это условие стало бы много более громоздким.)

$3^{*}$. Если $t \notin \operatorname{Max} T[\mathbf{X}]$ и $r \notin \operatorname{Max} T[\mathbf{Y}]$, то $j(r, t)=1$ равносильно тому, что

(a) $\forall r^{\wedge} b \in T[\mathbf{Y}] \exists t^{\wedge} a \in T[\mathbf{X}]\left(j\left(t^{\wedge} a, r^{\wedge} b\right)=1\right)$ и

(b) $\forall t^{\wedge} a \in T[\mathbf{X}] \exists r^{\wedge} b \in T[\mathbf{Y}]\left(j\left(t^{\wedge} a, r^{\wedge} b\right)=1\right)$.

В теории ZFC, например, для любых двух А-кодов $\mathbf{X}, \mathbf{Y}$ существует единственная бисимуляция $j: T[\mathbf{X}] \times T[\mathbf{Y}] \rightarrow\{0,1\}$, определенная так, что $j(t, r)=1$ равносильно $\mathrm{F}_{\mathbf{X}}(t)=\mathrm{F}_{\mathbf{Y}}(r)$. Но, в отличие от кодируемых множеств, бисимуляции существуют и в $\mathbf{n G B}$, так как построение бисимуляций не увеличивает $\in$-ранга над I!!

ЛЕмма 3.14 (nGB + пм-ограниченная Свертка). Для любых А-кодов $\mathbf{X}, \mathbf{Y} \in$ $\mathbf{H}$ существует единственная бисимулящия $j$.

ДокАзАтельство. Для $t \in T[\mathbf{X}]$ и $r \in T[\mathbf{Y}](t, r)$-функиией называется любая функция (класс) $j$, определенная на классе $Q_{t r}=\left\{\left\langle t^{\prime}, r^{\prime}\right\rangle \in T[\mathbf{X}] \times T[\mathbf{Y}]\right.$ : $\left.t \subseteq t^{\prime} \wedge r \subseteq r^{\prime}\right\}$ и удовлетворяющая пунктам $1^{*}, 2^{*}, 3^{*}$ определения 3.13 на $Q_{t r}$.

Предположим сначала, что коды $\mathbf{X}=\mathbf{x}$ и $\mathbf{Y}=\mathbf{y}$ принадлежат $\mathbf{h}$. Тогда $Q_{t r}$ - полумножество для любой пары $t \in T[\mathbf{x}], r \in T[\mathbf{y}]$. Через $S(t)$ обозначим утверждение: “для каждого $r \in T[\mathbf{y}]$ существует единственная $(t, r)$-функция $j_{t r}$ ". В предположении, что $\mathbf{x}, \mathbf{y}$ принадлежат $\mathbf{h},(t, r)$-функции являются полумножествами. Поэтому формула, определяющая $S(t)$, пм-ограничена, так что $S=\{t \in T: S(t)\}$ есть легитимный класс. Более того, $S$ индуктивен в смысле, указанном в доказательстве леммы $3.7: f_{t r}$ компонуется из функций 
$f_{t^{\wedge} a, r^{\wedge} b}$ для всевозможных $t^{\wedge} a \in T[\mathbf{x}], r^{\wedge} b \in T[\mathbf{y}]$ при помощи $1^{*}, 2^{*}, 3^{*}$ из определения 3.13. Значит, $S$ содержит $\Lambda$. Таким образом, имеется единственная $(\Lambda, \Lambda)$-функция, она же бисимуляция для кодов $\mathbf{x}, \mathbf{y}$.

Теперь общий случай: один или оба кода $\mathbf{X}, \mathbf{Y}$ принадлежат $\mathbf{H} \backslash \mathbf{h}$. По определению $\mathbf{H}$, классы $Q_{t r}$ являются полумножествами, когда $t \neq \Lambda$ и $r \neq \Lambda$. Очевидная модификация предыдущего рассуждения приносит в этом случае существование единственной $(t, r)$-функции $j_{t r}$ для любой пары $t \in T[\mathbf{X}] \backslash\{\Lambda\}$ и $r \in T[\mathbf{Y}] \backslash\{\Lambda\}$. Но тогда единственная $(\Lambda, \Lambda)$-функция компонуется из функций $f_{\langle a\rangle,\langle b\rangle}$ для всевозможных одночленных последовательностей $\langle a\rangle \in T[\mathbf{X}],\langle b\rangle \in$ $T[\mathbf{Y}]$. Лемма 3.14 доказана.

Эта единственная, для $\mathbf{X}, \mathbf{Y} \in \mathbf{H}$, бисимуляция будет обозначаться $\mathbf{j} \mathbf{X Y}$.

3d. Кумулятивное расширение нестандартного универсума классов. Мъ продолжаем рассуждать в $\mathbf{n G B}$ в рамках соглашения 3.5.

Следующее определение вводит st- $\in$-формулы, определяющие те отношения между А-кодами, которые адекватно выражают принадлежность и равенство между кодируемыми объектами, так, как если бы последние действительно существовали.

ОПРЕДЕЛЕНИЕ 3.15 .

1) $\mathbf{X}^{\mathrm{a}}=\mathbf{Y}$ есть st- $\in$-формула $\mathbf{X}, \mathbf{Y} \in \mathbf{H} \wedge \mathbf{j}_{\mathbf{X Y}}(\Lambda, \Lambda)=1$.

2) $\mathbf{X}^{\mathrm{a}} \in \mathbf{Y}$ есть st- $\in$-формула, говорящая, что $\mathbf{X}, \mathbf{Y} \in \mathbf{H}$ и выполнено одно из двух: либо а) $\mathbf{X}={ }^{\mathrm{a}} x$ и $\mathbf{Y}={ }^{\text {а }} Y$ для некоторых множества $x$ и класса $Y$ с $x \in Y$, либо b) $T[\mathbf{Y}] \neq\{\Lambda\}$ и существует $b \in \operatorname{Min} T[\mathbf{Y}]$ такое, что $\mathbf{j}_{\mathbf{X Y}}(\Lambda,\langle b\rangle)=1$.

3) ${ }^{\text {a }}$ st $\mathbf{X}$ есть st- $\in$-формула $\exists^{\text {st }} Y\left(\mathbf{X}={ }^{\mathrm{a}} Y\right)$.

ПРЕДЛОЖЕНИЕ 3.16 (nGB+пм-ограниченная Свертка). Отношения ${ }^{\mathrm{a}} \in{ }^{\mathrm{a}} \mathrm{st}$, $\mathrm{a}=$ могут быть определены пм-ограниченными бормулами.

ДокАЗАТЕЛЬСтво. Если рассматриваются только коды из $\mathbf{h}$, которые по определению являются полумножествами, то все относящиеся к делу бисимуляции - также полумножества, так что пм-ограниченность определений здесь очевидна.

В общем случае (произвольные коды из $\mathbf{H}$ ) коды и бисимуляции могут быть “большими" классами (не полумножествами). Это, однако, можно обойти. Например, в определении $\mathbf{X}^{\mathrm{a}}=\mathbf{Y}$ в определении $\left.3.15,1\right)$ формула $\mathbf{j} \mathbf{X Y}(\Lambda, \Lambda)=1$ равносильна, в случае, когда $T_{\mathbf{X}} \neq\{\Lambda\}$ и $T_{\mathbf{Y}} \neq\{\Lambda\}$, следующей формуле:

$$
\begin{aligned}
\forall a & \in \operatorname{Min} T_{\mathbf{X}} \exists b \in \operatorname{Min} T_{\mathbf{Y}}\left(\mathbf{j}_{\left.\left.\mathbf{X}\right|_{a} \mathbf{Y}\right|_{b}}(\Lambda, \Lambda)=1\right) \\
& \wedge \forall b \in \operatorname{Min} T_{\mathbf{Y}} \exists a \in \operatorname{Min} T_{\mathbf{X}}\left(\mathbf{j}_{\left.\left.\mathbf{X}\right|_{a} \mathbf{Y}\right|_{b}}(\Lambda, \Lambda)=1\right) .
\end{aligned}
$$

Однако, в соответствии с определением $\mathbf{H}$, коды $\left.\mathbf{X}\right|_{a},\left.\mathbf{Y}\right|_{b}$ здесь принадлежат $\mathbf{h}$, что сводит задачу к первому случаю. Предложение 3.16 доказано.

Отношения $^{\mathrm{a}}=$ и $^{\mathrm{a}} \in$ имеют простой смысл в ZFC, где кодируемые множества $A_{\mathbf{X}}$ существуют: $\mathbf{X}^{\mathbf{a}}=\mathbf{Y}$ равносильно $A_{\mathbf{X}}=\mathbf{Y}$ и $\mathbf{X}^{\mathbf{a}} \in \mathbf{Y}$ равносильно $A_{\mathbf{X}} \in A_{\mathbf{Y}}$. В нестандартных теориях классов обращение к кодируемым объектам, вообще говоря, невозможно, однако мы можем рассмотреть структуры

$$
\mathfrak{H}=\left\langle\mathbf{H} ;{ }^{\mathrm{a}} \in,{ }^{\mathrm{a}}{ }^{\mathrm{st}} ;{ }^{\mathrm{a}}=\right\rangle \quad \text { и } \quad \mathfrak{h}=\left\langle\mathbf{h} ;{ }^{\mathrm{a}} \in \uparrow \mathbf{h},{ }^{\mathrm{a}} \text { st } \uparrow \mathbf{h} ;{ }^{\mathrm{a}}=\uparrow \mathbf{h}\right\rangle,
$$


т.е. наши кодированные кумулятивные расширения, как структуры st- $\in$-языка. Для экономии места достаточно элементарное доказательство следующего предложения оставляется читателю.

ПрЕДЛОЖЕНИЕ 3.17 (nGB+пм-ограниченная Свертка).

(i) ${ }^{\mathrm{a}}=-$ отношение эквивалентности на $\mathfrak{H}$, а отношения ${ }^{\mathrm{a}} \in u^{\mathrm{a}}{ }^{\mathrm{st}}{ }^{\mathrm{a}}=-$ инвариантны.

(ii) Для любого класса $X{ }^{\mathrm{a}} X \in \mathbf{h}$ равносильно тому, что $X$ - полумножеcmso.

(iii) Отображение $X \mapsto{ }^{\mathrm{a}} X$ является st- $\in$-изоморфизмом в том смысле, что для любых $X, Y$ выполнено:

$$
\begin{aligned}
X \in Y & \Leftrightarrow{ }^{\mathrm{a}} X{ }^{\mathrm{a}} \in{ }^{\mathrm{a}} Y, \\
\text { st } X & \Leftrightarrow{ }^{\mathrm{a}} \mathrm{st}^{\mathrm{a}} X, \\
X=Y & \Leftrightarrow{ }^{\mathrm{a}} X^{\mathrm{a}}={ }^{\mathrm{a}} Y \Leftrightarrow{ }^{\mathrm{a}} X={ }^{\mathrm{a}} Y .
\end{aligned}
$$

(iv) (a) $E$ сли $T[\mathbf{Y}] \neq\{\Lambda\}, \operatorname{mo} \mathbf{X}^{\mathrm{a}} \in \mathbf{Y} \Leftrightarrow \exists b \in \operatorname{Min} T[\mathbf{Y}]\left(\mathbf{X}^{\mathrm{a}}=\left.\mathbf{Y}\right|_{b}\right)$.

(b) Ecли $\mathbf{Y}={ }^{\mathrm{a}} Y$, mo $\mathbf{X}{ }^{\mathrm{a}} \in \mathbf{Y} \Leftrightarrow \exists x \in Y\left(\mathbf{X}={ }^{\mathrm{a}} x\right)$.

(v) (a) Если $y \in \mathbb{I}$, mо $\mathbf{X}^{\mathrm{a}}={ }^{\mathrm{a}} y$ равносильно тому, что $\mathbf{X}={ }^{\mathrm{a}} y$.

(b) Если $Y \notin \mathbb{I}$, то $\mathbf{X}^{\mathrm{a}}={ }^{\mathrm{a}} Y$ равносильно тому, что $\left|T_{\mathbf{X}}\right|^{\mathbb{S}}=1$ и $Y=$ $\left\{\mathbf{X}(a): a \in \operatorname{Min} T_{\mathbf{X}}\right\}$.

Таким образом, согласно (i), структуры $\mathfrak{H}, \mathfrak{h}$ относится к типу инвариантных (см. конец п. 1a) st- $\in$-структур: равенство интерпретируется через отношение эквивалентности ${ }^{\mathrm{a}}=$, а отношения ${ }^{\mathrm{a}} \in$ и $^{\mathrm{a}} \mathrm{st}{ }^{\mathrm{a}}=-$-инвариантны. В отличие от ситуации, рассмотренной в замечании 1.6, здесь не видно никакой возможности адекватно заменить классы эквивалентности (которые здесь сами могут состоять из собственных классов) множествами или хотя бы "законными" классами множеств.

ОПРЕДЕЛЕНИЕ 3.18 (ср. с определением 1.15).

Для любой инвариантной st- $\in$-структуры $\mathfrak{X}=\left\langle X ; \in_{\mathfrak{X}}, \mathrm{st}_{\mathfrak{X}} ;=_{\mathfrak{X}}\right\rangle$ определим области $(\mathbb{H})^{\mathfrak{X}},(\mathbb{S})^{\mathfrak{X}},(\mathbf{S})^{\mathfrak{X}},(\mathbb{I})^{\mathfrak{X}},(\mathbf{E})^{\mathfrak{X}},(\mathbf{I})^{\mathfrak{X}},(\mathbf{H})^{\mathfrak{X}}$ (части $\left.X\right)$ в $\mathfrak{X}$ согласно определению 2.4. Пусть $\mathfrak{Y}=\left\langle Y ; \in_{\mathfrak{Y}}, \mathrm{st}_{\mathfrak{Y}}\right\rangle$ - еще одна st- $\in$-структура. Инъекция $\pi: Y \rightarrow X$ называется

1) st- $\in$-вложением $\mathfrak{Y}$ в $\mathfrak{X}$, когда имеют место эквивалентности $y \in \in_{Y} y^{\prime} \Leftrightarrow$ $\pi(y) \in_{X} \pi\left(y^{\prime}\right)$ и $\operatorname{st}_{Y} y \Leftrightarrow \operatorname{st}_{X} \pi(y)$ для всех $y, y^{\prime} \in Y$

2) st- $\in$-вложением $\mathfrak{Y}$ в $\mathfrak{X} \mathbb{I}$-mипа, когда, дополнительно к 1), полный образ $\operatorname{ran} \pi=\{\pi(y): y \in Y\}$ совпадает с $(\mathbb{I})^{\mathfrak{X}}$ с точностью до $=\mathfrak{X} ; ;^{30}$

3) st- $\in$-вложением $\mathfrak{Y}$ в $\mathfrak{X} \mathbf{E}$-типа, когда, дополнительно к 1), полный образ $\operatorname{ran} \pi$ совпадает с $(\mathbf{E})^{\mathfrak{X}}$ с точностью до $=\mathfrak{X}$.

В этом случае st- $\in$-структура $\mathfrak{X}$ будет называться расширением I-muna, соответственно E-muпа, структуры $\mathfrak{Y}$ (посредством отображения $\pi$ ).

Следующее утверждение раскрывает природу областей $(\mathbb{H})^{\mathfrak{X}},(\mathbb{S})^{\mathfrak{X}}$ и т. д. для структур $\mathfrak{h}$ и $\mathfrak{H}$. Доказательство не составляет труда, хотя в некоторых деталях довольно трудоемко и требует обращения к пунктам (iii), (iv), (v) предложения 3.17. Обозначения $\mathbb{S}, \mathbb{I}, \mathbf{S}, \mathbf{I}, \mathbf{E}$ без релятивизации соответствуют в

\footnotetext{
30 Части $A, B$ некоторой области $D$, где действует какое-то отношение эквивалентности $\approx$, совпадают с точностъю до $\approx$, когда $\forall a \in A \exists b \in B(a \approx b)$ и $\forall b \in B \exists a \in A(a \approx b)$.
} 
предложении 3.19 и следствии 3.20 определению 2.4 в базовом нестандартном универсуме теории $\mathbf{n G B + п м - о г р а н и ч е н н а я ~ С в е р т к а . ~}$

ПРЕДЛОЖЕНИЕ 3.19 (nGB + пм-ограниченная Свертка).

(i) $(\mathbb{H})^{\mathfrak{H}}$ совпадает $c \mathbf{h}$ с точностъю до ${ }^{\mathrm{a}}=$, другими словами, $\mathfrak{H}$ является расширением структуры $\mathfrak{h}$ посредством добавления классов к ее облаcmu $\mathbf{h}$.

(ii) $(\mathbb{S})^{\mathfrak{H}}=(\mathbb{S})^{\mathfrak{h}}=\left\{{ }^{\mathrm{a}} x: x \in \mathbb{S}\right\} u(\mathbb{I})^{\mathfrak{H}}=(\mathbb{I})^{\mathfrak{h}}=\left\{{ }^{\mathrm{a}} x: x \in \mathbb{I}\right\}$.

(iii) $(\mathbf{S})^{\mathfrak{H}}=\left\{{ }^{\mathrm{a}} X: X \in \mathbf{S}\right\}, a(\mathbf{I})^{\mathfrak{H}}$ coвпадает $c\left\{{ }^{\mathrm{a}} X: X \in \mathbf{I}\right\}$ с точностью $\partial o^{\mathrm{a}}=$.

(iv) $(\mathbf{E})^{\mathfrak{H}}$ совпадает $c\left\{{ }^{\mathrm{a}} X: X \in \mathbf{E}\right\}$ с точностью до ${ }^{\mathrm{a}}=$.

Следующий результат сразу вытекает из предложений 3.17 и 3.19 .

СЛЕДСтвИЕ 3.20 (nGB+пм-ограниченная Свертка). Отображение $X \mapsto{ }^{\mathrm{a}} X$ является st- $\in$-вложением E-типа всего базового универсума $\mathbf{n G B}$ в $\mathfrak{H}$.

Ограниченное вложение $x \mapsto{ }^{\mathrm{a}} x, x \in \mathbb{I}$, является st- $\in$-вложением I-типа структуры $\mathfrak{I}=\langle\mathbb{I} ; \in \in$, st $\rangle$ в $\mathfrak{H}$.

3е. Свойства кумулятивного расширения. Здесь доказывается следующая центральная теорема.

TEOPEMA 3.21 .

(i) Структура h есть интерпретация $\mathbf{H S T}^{+}$в $\mathbf{n G B}^{+}$, m.е. в $\mathbf{n G B}^{+}$выводима релятивизация $\Phi^{\mathfrak{h}}$ любой аксиомы $\Phi$ теории $\mathbf{H S T} \kappa \mathfrak{h}$.

(ii) Структура $\mathfrak{H}$ является интерпретацией теории $\mathbf{G B} / \mathbf{H S T}^{+}$в $\mathbf{n G B}^{+}$, а также интерпретацией теории $\mathbf{K M} / \mathbf{H S T}^{+}$в $\mathbf{n K M}^{+}$.

ДокАзАтельство. (i) Громоздкость рассуждений, сводящихся к проверке всех необходимых фактов (см. §5.4 в [25] или часть II статьи [21]), не позволяет изложить здесь полное доказательство. Впрочем, большая часть аргументов довольно элементарна, так что не составляет труда вывести, например, релятивизации аксиом Выбор стандартного размера, Зависимого выбора, Насыщенности, I-образа, $\Sigma_{2}^{\text {st }}$-множества теории $\mathbf{H S T}^{+}$при помощи одноименных дополнительных аксиом из $\mathbf{n G B}^{+}$, используя, при необходимости, предложение 3.17 и следующую лемму 3.22 .

Определим, для любого класса $X, \operatorname{FAM}(X)=\{X[a]: a \in \operatorname{dom} X\}$, где, напомним, $X[a]=\{b:\langle a, b\rangle \in X\}$. Строго говоря, $\operatorname{FAM}(X)$ - это совокупности классов, т.е. незаконные образования в наших теориях классов. Однако читатель без труда обнаружит, что все явные и подразумеваемые вхождения $\operatorname{FAM}(\cdot)$ ниже - лишь удобная фигура речи, легко устраняемая через редукцию к определению.

ЛЕмма $3.22\left(\mathbf{n G B}^{+}\right)$. Для любого класса $X$ c $\operatorname{FAM}(X) \subseteq \mathbf{h}$ найдется код

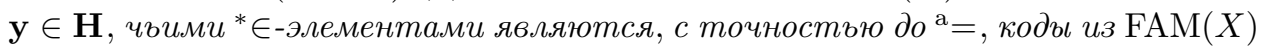
и только они. Если же $X$ - полумножество, то такой коду можно выбрать $u \in \mathbf{h}$.

ДоКАЗАТЕЛЬСТВО. Если $X=\varnothing$, то $\mathbf{y}={ }^{\text {a }} \varnothing-$ искомый код, поэтому считаем, что $X \neq \varnothing$. Тогда и $D=\operatorname{dom} X \neq \varnothing$. Определим А-код у так, что $T[\mathbf{y}]=$ $\{\Lambda\} \cup\left\{a^{\wedge} t: a \in D \wedge t \in T[X[a]]\right\}$ и $\mathbf{y}\left(a^{\wedge} t\right)=X[a](t)$ всякий раз, когда $a \in D$ 
и $t \in \operatorname{Max} T[X[a]]$. Понятно, что $\operatorname{Min} T[\mathbf{y}]=D$ и $\left.\mathbf{y}\right|_{a}=X[a]$ для любого $a \in D$. Поэтому ${ }^{*} \in$-элементами у являются, с точностью до ${ }^{a}=$, коды из $\operatorname{FAM}(X)$ и только они. Если $\mathbf{y}$ - регулярный код, то это решает задачу: $\mathbf{y}$ принадлежит $\mathbf{H}$. Более того, если $X$ - полумножество, то этот код у принадлежит $\mathbf{h}$.

Если $\mathbf{y}$ - нерегулярный код, то, согласно определению и поскольку все коды $\left.\mathbf{y}\right|_{a}=X[a]$ с $a \in D=\operatorname{Min} T[\mathbf{y}]$ регулярны, мы имеем $T[\mathbf{y}]=\langle\lambda\rangle \cup\{\langle a\rangle: a \in D\}$, и для каждого $a \in \operatorname{dom} X$ найдется множество $x_{a}$ такое, что $\left.\mathbf{y}\right|_{a}={ }^{\mathrm{a}}\left(x_{a}\right)$, т.е. $\mathbf{y}(\langle a\rangle)=x_{a}$, причем $y=\left\{x_{a}: a \in D\right\}$ является множеством. В этой ситуации лемма доказывается при помощи кода ${ }^{\mathrm{a}} y$. Лемма 3.22 доказана.

Доказанная лемма позволяет без труда доказать Выделение в $\mathfrak{h}$ в st- $\in$-языке, т.е. то свойство, из-за которого, в какой-то мере, и проводится данное построение. Именно, возьмем произвольный код $\mathbf{X} \in \mathbf{h}$ и произвольную st- $\in$-формулу $\Phi(x)$ с кодами из $\mathbf{h}$ в качестве параметров. Согласно лемме 3.22 , достаточно найти полумножество $X$ такое, что $\operatorname{FAM}(X) \subseteq \mathbf{h}$ и $\operatorname{FAM}(X)$ содержит, с точностью до ${ }^{a}=$, каждый код $\mathbf{x}^{\mathrm{a}} \in \mathbf{X}$, удовлетворяющий $\Phi(\mathbf{x})^{\mathfrak{h}}$, и не содержит ничего больше.

В случае, когда код $\mathbf{X}$ имеет вид $\mathbf{X}={ }^{\text {а }} Y$ для некоторого $Y \in \mathbb{I}$, рассмотрим совокупность кодов $\mathscr{X}=\left\{{ }^{\mathrm{a}} y: y \in Y \wedge \Phi\left({ }^{\mathrm{a}} y\right)^{\mathfrak{h}}\right\}-$ т.е., согласно предложению $3.17(\mathrm{iv})(\mathrm{b})$, всех ${ }^{\mathrm{a}} \in$-элементов ${ }^{\mathrm{a}} Y$, удовлетворяющих $\Phi(\cdot)^{\mathfrak{h}}$. Отметим, что формула $\Phi(x)^{\mathfrak{h}}$ (со свободной переменной $x$ ) является пм-ограниченной комбинацией отношений ${ }^{\mathrm{a}} \in,{ }^{\mathrm{a}} \mathrm{st},{ }^{\mathrm{a}}=$, поскольку ее кванторы ограничены совокупностью $\mathbf{h}$, состоящей из полумножеств. Поэтому $X=\{\langle y, b\rangle: y \in Y \wedge$ $\left.b \in{ }^{\mathrm{a}} y \wedge \Phi\left({ }^{\mathrm{a}} y\right)^{\mathfrak{h}}\right\}$ является классом (см. предложение 3.16), а тогда и полумножеством. Однако $\mathscr{X}=\operatorname{FAM}(X)$, что и требовалось. Если же $T[\mathbf{X}] \neq\{\Lambda\}$, то искомый результат выводится из рассмотрения совокупности кодов $\left\{\left.\mathbf{X}\right|_{a}\right.$ : $\left.a \in \operatorname{Min} T \wedge \Phi\left(\left.\mathbf{X}\right|_{a}\right)^{\mathfrak{h}}\right\}$ (и пункта (iv)(a) предложения 3.17 вместо пункта (iv)(b)).

Проверим еще и аксиому Собирания в $\mathfrak{h}$. Пусть $\mathbf{X} \in \mathbf{h}, T[\mathbf{X}] \neq\{\Lambda\}$, а $\Phi(x, y)$ является st- $\in$-формулой с кодами из $\mathbf{h}$ в качестве параметров. Согласно лемме 3.22 и предложению $3.17(\mathrm{iv})(\mathrm{a})$, достаточно найти полумножество $X$ такое, что $\operatorname{FAM}(X) \subseteq \mathbf{h}$ и

$$
\exists \mathbf{y} \in \mathbf{h} \Phi\left(\left.\mathbf{X}\right|_{b}, \mathbf{y}\right)^{\mathfrak{h}} \Rightarrow \exists \mathbf{y} \in \operatorname{FAM}(X)\left(\Phi\left(\left.\mathbf{X}\right|_{b}, \mathbf{y}\right)^{\mathfrak{h}}\right)
$$

выполнено для каждого А-кода $\left.\mathbf{X}\right|_{b}$, где $b \in \operatorname{Min} T[\mathbf{X}]$. Но существование такого $X$ легко следует из схемы Собирания полумножеств.

(ii) Выполнение $\left(\mathbf{H S T}^{+}\right)^{\text {sets }}$ в $\mathfrak{H}$ следует из (i), поскольку $\mathfrak{h}$ является универсумом множеств в $\mathfrak{H}$ по предложению 3.19(i). Далее, для вывода $\mathbf{G B}^{\text {st }}$, Стандартизации классов и аксиомы $\mathbf{S}=\mathbf{S}^{\text {loc }}$ для классов $X \subseteq \mathbb{I}$ в $\mathfrak{H}$ достаточно заметить, что в силу предложений $3.17\left(\right.$ iii) и $3.19(\mathrm{iv})$ область $(\mathbf{E})^{\mathfrak{H}}$ st- $\in$-изоморфна базовому универсуму $\mathbf{n G B}^{+}$(или $\mathbf{n} \mathbf{K M}^{+}$), где эти аксиомы выполнены. Экстенсиональность в $\mathfrak{H}-$ простое упражнение. Остается проверить Собирание и Свертку для предикативных st- $\in$-формул в GB-случае, а также Непредикативную Свертку и Собирание Классов для всех st- $\in$-формул в КМ-случае.

Свертка и Непредикативная Свертка. Достаточно найти "класс всех множеств" в $\mathfrak{H}$, т.е. такой код $\mathbf{Y} \in \mathbf{H}$, что $\mathbf{x}^{\mathrm{a}} \in \mathbf{Y}$ для всех $\mathbf{x} \in \mathbf{h}$. В самом деле, имея такой код $\mathbf{Y}$, мы легко завершаем доказательство, следуя доказательству Выделения 
в части (i). Чтобы определить искомый код $\mathbf{Y}$, положим

$$
P=\left\{p \in \mathbf{C}_{0}: E_{p} \in \mathbf{h}\right\} \quad \text { и } \quad X=\left\{\langle p, x\rangle: p \in P \wedge x \in E_{p}\right\} .
$$

Принадлежность к h, очевидно, выражается пм-ограниченной формулой. Поэтому, даже в теории $\mathbf{n G B}^{+}, P$ и $X$ являются корректно определенными классами. Однако по аксиоме $\Sigma_{2}^{\text {st }}$-полумножеств каждый код $\mathbf{x} \in \mathbf{h}$ удовлетворяет $\mathbf{x}=E_{p}=X[p]$, так что $\operatorname{FAM}(X)$ содержит все коды из $\mathbf{h}$. Остается применить лемму 3.22 .

Собирание (предикативное) - аналогично выводу Собирания в части (i).

Собирание Классов. Предполагая $\mathbf{n} \mathbf{K M}^{+}$, мы должны доказать в $\mathfrak{H}$ следующее:

$$
\forall d \exists C \exists r \forall a \in d(\exists Y \Phi(a, Y) \Rightarrow \exists x \in r \Phi(a, C[x])),
$$

где $\Phi(x)$ - любая st- $\in$-формула с кодами из $\mathbf{H}$ в роли параметров. Пусть $\mathbf{d} \in \mathbf{h}$. Как и в доказательстве Выделения в части (i), имеется полумножество $X$ такое, что $\operatorname{FAM}(X)=\left\{\boldsymbol{a} \in \mathbf{h}: \boldsymbol{a}^{\mathrm{a}} \in \mathbf{d}\right\}$ с точностью до ${ }^{\mathrm{a}}=$. Положим $\delta=\operatorname{dom} X$, так что $\operatorname{FAM}(X)=\{X[\xi]: \xi \in \delta\}$.

Применяя Собирание Классов в базовом универсуме $\mathbf{n} \mathbf{K M}^{+}$, получаем такие класс $W \subseteq \mathbb{I}$ и множество $w \in \mathbb{I}$, что для каждого $\xi \in \delta$ существует $x \in w$, для которого $\mathbf{W}_{x}=W[x]-$ код в $\mathbf{H}$ и если выполнено $(\exists Y \Phi(X[\xi], Y))^{\mathfrak{H}}$, то также выполнено $\Phi\left(X[\xi], \mathbf{W}_{x}\right)^{\mathfrak{H}}$. Лемма 3.22 приносит код $\mathbf{C} \in \mathbf{H}$, который с точностью до ${ }^{\mathrm{a}}={ }^{\mathrm{a}} \in$-содержит коды вида ${ }^{\mathrm{a}}\left\langle{ }^{\mathrm{a}} x, \mathbf{z}\right\rangle,{ }^{31}$ где $\eta \in w$ и $\mathbf{z}^{\mathrm{a}} \in \mathbf{W}_{\eta}$, и ничего кроме них. Тогда $\mathbf{W}_{x}=\mathbf{C}\left[{ }^{\mathrm{a}} x\right]$ выполнено в $\mathfrak{H}$ для каждого $x \in w$.

Теперь предположим, что $\boldsymbol{a} \in \mathbf{h}, \boldsymbol{a}^{\mathrm{a}} \in \mathbf{d}$ и выполнено $(\exists Y \Phi(\boldsymbol{a}, Y))^{\mathfrak{H}}$. По определению существует $\xi \in \delta$, для которого $\boldsymbol{a}=X[\xi]$, и, следовательно, найдется $x \in w$ такое, что выполнено $\Phi\left(\boldsymbol{a}, \mathbf{W}_{x}\right)^{\mathfrak{H}}$, а тогда и $\Phi\left(\boldsymbol{a}, \mathbf{C}\left[{ }^{\mathrm{a}} x\right]\right)^{\mathfrak{H}}$. Следовательно, $\mathbf{C}$ и $r={ }^{\mathrm{a}} w$ доказывают соотношение (13) в $\mathfrak{H}$ для $d=\mathbf{d}$. Теорема 3.21 доказана.

Остается заметить, что, в соответствии со следствием 3.20, интерпретация теории $\mathbf{G B} / \mathbf{H S T}^{+}{ }_{\text {в }} \mathbf{n G B}^{+}$и теории $\mathbf{K M} / \mathbf{H S T}^{+}{ }_{\text {в }} \mathbf{n K M}^{+}$, даваемая структурой $\mathfrak{H}$ по теореме 3.21(ii), может быть названа интерпретащией E-типа в том смысле, что отображение $X \mapsto{ }^{\text {а }} X$ осуществляет st- $\in$-изоморфизм всего универсума $\mathbf{n G B}^{+}$на область $(\mathbf{E})^{\mathfrak{H}}$ всех классов внутренних множеств структуры $\mathfrak{H}$. А неформальное заключение из теоремы 3.21(iі) и следствия 3.20 состоит в том, что универсум $\mathbf{E}$ теории $\mathbf{n G B}^{+}$(соответственно $\mathbf{n} \mathbf{K M}^{+}$) расширяется до структуры $\mathfrak{H}$, удовлетворяющей $\mathbf{G B} / \mathbf{H S T}^{+}\left(\right.$соответственно $\mathbf{K M} / \mathbf{H S T}^{+}$), в которой этот универсум остается совокупностью $(\mathbf{E})^{\mathfrak{H}}$ всех классов внутренних множеств.

Аналогично, интерпретация, даваемая структурой $\mathfrak{h}$ по теореме 3.21(i), может быть названа интерпретацией II-muпа в том смысле, что отображение $x \mapsto{ }^{\text {a }} x$ осуществляет st- $\in$-изоморфизм BST-структуры $\mathfrak{I}=\{\mathbb{I} ; \in, \mathrm{st}\}$ (здесь: класса всех множеств в базовом универсуме $\mathbf{n G B}^{+}$) на область внутренних множеств $(\mathbb{I})^{\mathfrak{h}}$ структуры $\mathfrak{h}$. Это можно трактовать неформально как расширение структуры $\mathfrak{I}$ всех (внутренних) множеств в $\mathbf{n G B}^{+}$до структуры $\mathfrak{h}$, удовлетворяющей $\mathbf{H S T}^{+}$, в которой $\mathfrak{I}$ остается классом $(\mathbb{I})^{\mathfrak{h}}$ всех внутренних множеств.

\footnotetext{
${ }^{31}$ Под ${ }^{\mathrm{a}}\left\langle{ }^{\mathrm{a}} x, \mathbf{z}\right\rangle$ понимается код из $\mathbf{h}$, некоторым каноническим образом определяющий упорядоченную пару из указанных элементов в $\mathfrak{h}$.
} 
3f. Метаматематические следствия. Как и выше, интерпретации указанных видов ведут к утверждениям о консервативности в соответствующих формах.

\section{СЛЕДСТВИЕ 3.23.}

(i) Теория HST $^{+}$является I-консервативным (тогда и равнонепротиворечивым) расширением BST в том смысле, что любая st- $\in$-формула $\varphi$ въводима в BST тогда и только тогда, когда $\varphi^{\mathbb{I}}$ выводима в $\mathbf{H S T}^{+}$.

(ii) Теория GB/HST ${ }^{+}$является $\mathbf{E - к о н с е р в а т и в н ы м ~ р а с ш и р е н и е м ~} \mathbf{n G B}^{+}$ в том смысле, что любая st- $\in$ формула $\varphi$ выводима в $\mathbf{n G B}^{+}$тогда и только тогда, когда $\varphi^{\mathbf{E}}$ выводима в $\mathbf{G B} / \mathbf{H S T}^{+}$. То же самое для nары $\mathbf{K M} / \mathbf{H S T}^{+} u \mathbf{n K M}^{+}$.

ДокАЗАтельство. (ii) Допустим, что релятивизованная формула $\varphi^{\mathbf{E}}$ выво-

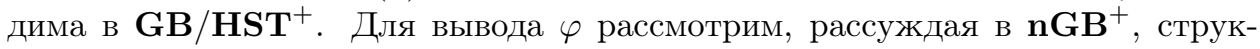
туру $\mathfrak{H}$. Согласно теореме $3.21, \varphi^{\mathbf{E}}$ выполнено в $\mathfrak{H}$. Но $(\mathbf{E})^{\mathfrak{H}}$ доказуемо st- $\in$ изоморфно базовому универсуму $\mathbf{n G B}^{+}$согласно следствию 3.20 , что и завершает вывод $\varphi$.

(i) Если $\varphi^{\mathbb{I}}$ выводится в $\mathbf{H S T}^{+}$, то $\varphi^{\mathbb{I}}$ следует из $\mathbf{G B} / \mathbf{H S T}^{+}$по теореме 3.4 , следовательно, и из $\mathbf{n G B}^{+}$согласно (ii). (Релятивизация sets ${ }^{\mathbf{E}}$ для формулы, релятивизованной к II, ничего не меняет.) Значит, $\varphi$ доказывается в BST согласно следствию 2.21 .

Диаграммы 3 и 4 представляют главные метаматематические результаты, связывающие теории BST и $\mathbf{H S T}^{+}$между собой и с соответствующими нестандартными теориями классов. На диаграммах $A \longrightarrow B$ означает, что $A$ так или иначе сводится к $B$. Диаграмма 3 представляет естественные сводимости "меньшей" теории к "большей". Вертикальные стрелки обозначают интерпретацию посредством релятивизации к $\mathbb{I}$ или $\mathbf{E}$ (см. предложения $3.1,3.3)$. Стрелки, помеченные sets, обозначают очевидную интерпретацию посредством релятивизации к подуниверсуму множеств (в универсуме классов). Стрелки, помеченные $\subset$, символизируют тот факт, что $\mathbf{n G B}^{+}$и $\mathbf{G B} / \mathbf{H S T}^{+}$суть подтеории теорий $\mathbf{n K M}^{+}$и соответственно $\mathbf{K M} / \mathbf{H S T}^{+}$.
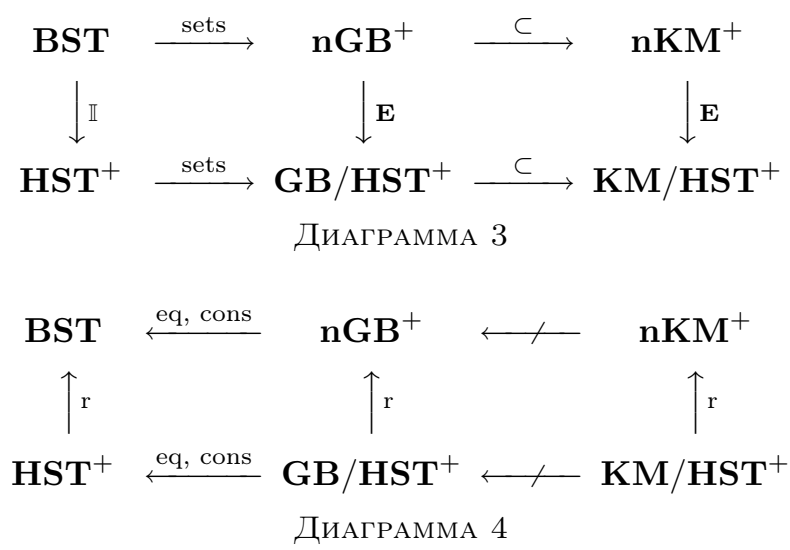
На диаграмме 4 (обратные сводимости) перечеркнутые стрелки обозначают сводимости, не имеющие места по той причине, что непредикативные теории классов строго сильнее в смысле непротиворечивости, чем предикативные. (В отношении пары $\mathbf{G B} / \mathbf{H S T}^{+}, \mathbf{K M} / \mathbf{H S T}^{+}$можно сослаться на теорему 3.4.) Вертикальные стрелки, помеченные r, обозначают интерпретации из теоремы 3.21 и вытекающие из них утверждения о консервативности и непротиворечивости из следствия 3.23 .

Стрелки, помеченные eq, cons (от equiconsistency, conservativity), означают, что сводимость теории классов к теории множеств имеет место по отношению к свойствам sets-консервативности и непротиворечивости, но не имеет места для интерпретируемости. Этот результат дается теоремами 2.6 и 3.4.

\section{3g. Как избавиться от предположения о существовании оболочки} из классов. Теорема 3.21(i), утверждающая, как мы видели, возможность расширения "внутреннего" универсума II теории BST до "внешнего" универсума теории $\mathbf{H S T}^{+}$, предполагает, что данный универсум II есть класс всех множеств в более широком универсуме классов теории $\mathbf{n G B}^{+}$. Это предположение, полезное и обоснованное в свете соответствующих результатов о теориях классов, может быть, однако, элиминировано в контексте именно теоремы 3.21(i), что и является целью этого короткого пункта.

В сущности, задача состоит в том, чтобы как-то построить, на базе универсума II теории BST, более широкий универсум, по своим свойствам подобный совокупности всех полумножеств в $\mathbf{n G B}^{+}$, на основе которого, собственно, и была построена интерпретация $\mathfrak{h}$. Оказывается, что для этого достаточно присоединить к II все совокупности вида $E_{p}$, где $p \in \mathbf{C}_{0}$. (Обозначения см. в определении 2.13 , но понимаются они уже на основе универсума II теории BST.)

Строго говоря, речь идет о следующем. Мы определяем, в BST, ${ }^{\mathbf{e}} x$ как функцию, заданную на одноэлементном множестве $\{\langle 0,0\rangle\}$ условием ${ }^{\mathbf{e}} x(0,0)=$ $x$. Понятно, что ${ }^{\mathbf{e}} x \in \mathbf{C}_{0}$ и $E_{\mathbf{e}_{x}}=x$ для каждого $x$. Обозначим через $\mathbf{C}$ совокупность всех кодов $p \in \mathbf{C}_{0}$ таких, что либо $p={ }^{\mathbf{e}} x$ для некоторого $x \in \mathbb{I}$, либо $E_{p}$ не является множеством (в $\mathbb{I}$ ). Это ограничение обеспечивает однозначность кодировки уже имеющихся множеств. Далее, мы рассматриваем в BST реляционную структуру $\mathfrak{C}=\left\langle\mathbf{C} ;{ }^{\mathbf{e}} \in,^{\mathbf{e}} \mathrm{st},{ }^{\mathbf{e}}=\right\rangle$, где ${ }^{\mathbf{e}} \in,^{\mathbf{e}_{\mathrm{st}}},{ }^{\mathbf{e}}=$ суть отношения на $\mathbf{C}$, определенные следующим образом:

$$
\begin{array}{ll}
p^{\mathbf{e}}=q, & \text { когда } p, q \in \mathbf{C} \wedge \forall z\left(z \in E_{p} \Leftrightarrow z \in E_{q}\right) ; \\
p^{\mathbf{e}} \in q, & \text { когда } p, q \in \mathbf{C} \wedge \exists z\left(p=\mathbf{e}_{z} \wedge z \in E_{q}\right) ; \\
\mathbf{e}_{\text {st }} p, & \text { когда } \left.\exists^{\text {st }} z\left(p={ }^{\mathbf{e}} z\right), \quad \text { (отсюда следует } p \in \mathbf{C}\right) .
\end{array}
$$

Понятно, что структуру $\mathfrak{C}$ можно рассматривать как расширение универсума II теории BST при помощи всех "полумножеств" $E_{p}, p \in \mathbf{C}$; само расширение дается отображением $x \mapsto\left[{ }^{\mathbf{e}} x\right]_{\mathbf{e}}=$. Это расширение хорошо изучено в литератуpe. В частности, в [25; гл. 5] (см. также более ранние работы [21], [22]) показано, что $\mathfrak{E}$ является интерпретацией, в BST, теории элементарных внешних множеств EEST, т.е. теории в st- $\in$-языке, которая включает $\mathbf{B S T}^{\text {int }}$, а также аксиомы Экстенсиональности, Объединения, Выделения в st- $\in$-языке, Транзитивности и аксиому $\forall x \exists p\left(x=E_{p}\right)$, т.е. аналог наших аксиом $\Sigma_{2}^{\text {st }}$-полумножеств 
теории $\mathbf{n G B}^{+}$и $\Sigma_{2}^{\mathrm{st}}$-множеств теории $\mathbf{H S T}^{+}$. Это, в общем, достаточно простой результат: дело в том, что st- $\in$-Выделение, ключевой факт, без труда выводится при помощи теоремы 1.19. Таким образом, структура $\mathfrak{C}$ является интерпретацией EEST в BST.

C другой стороны, теория EEST достаточно сильна для доказательства тех фактов, которые теория $\mathbf{n G B}^{+}$влечет для универсума полумножеств, включая также дополнительную аксиому $\Sigma_{2}^{\text {st }}$-полумножеств. Это позволяет провести, в EEST, ту конструкцию кумулятивного расширения универсума, которая проведена выше на базе класса всех полумножеств теории $\mathbf{n G B}^{+}$. Это построение приводит к структуре $\mathfrak{h}$ из (12) на с. 101, которая удовлетворяет теореме 3.21 (с заменой $\mathbf{n G B}^{+}$на EEST).

Суперпозиция же этих двух расширений (посредством $\mathfrak{C}$ и посредством $\mathfrak{h}$ ) приносит расширение универсума $\mathbb{I}$ теории $\mathbf{B S T}$ до универсума $\mathbf{H S T}^{+}$, где $\mathbb{I}$ остается классом всех внутренних множеств. Строгая формулировка такова.

Теорема 3.24. В теории BST можно определить инвариантную st- $\in$ структуру, которая является интерпретацией I-типа (в смысле наличия соответствуюшего вложения) теории $\mathbf{H S T}^{+}$в $\mathbf{B S T} u$, доказуемо в $\mathbf{B S T}$, pacширением I-типа универсума всех множеств.

Исторические и библиографические замечания к § 3. О нестандартных теориях с Выделением в st- $\in$-языке, т.е. о теориях "внешнего" типа, см. книгу [25; гл. 8]. Сюда относятся аксиоматические схемы, предложенные К. Хрбачеком [10], [11], Т. Каваи [12], [13], Д. Баллардом и К. Хрбачеком [17]. Теория множеств Хрбачека HST в окончательном виде построена в [21] на основе ранней работы Хрбачека [10]. Систематическое исследование теоретико-множественного универсума HST произведено в книге В. Г. Кановея и М. Реекена [25; гл. 1].

Определение 3.2 и определение, содержащееся в формуле (12), предложение 3.16, теорема 3.21 и следствие 3.23 получены В. Г. Кановеем. Эти результаты рассматриваются как главные в этом параграфе.

Метод расширения универсума BST до более широкого универсума HST (фактически, до универсума $\mathbf{H S T}^{+}$), с промежуточным этапом, связанным с теорией EEST, как в п. 3g, был предложен в [21], [22]. Шаг BST $\rightarrow$ EEST основан на кодировке через $E_{p}$ и теореме 1.19 . Кодировка кумулятивного построения, как в п. 3с, лежащая в основе шага EEST $\rightarrow$ HST, известна с 1960-х годов, когда она была использована для интерпретации теорий типа ZFC без аксиомы степени в арифметике второго порядка. В контексте нестандартного анализа эта кодировка использована в [21], [22], [25], в несколько иной форме в [10]. Сам термин "бисимуляция" распространен в некоторых областях комбинаторики и теории систем; мы взяли его из книги [56], где бисимуляции использованы для исследования нестандартных теорий с аксиомами антифундирования.

Начиная не с BST, а с теорий классов типа nGB, мы уже имеем "внешние" множества $X \subseteq \mathbb{I}$ в виде подклассов множеств (т.е. полумножеств), так что нет необходимости в шаге, аналогичном BST $\rightarrow$ EEST. Поэтому доказательство нашей ключевой теоремы 3.21 сводится к кодировке кумулятивного построения. 


\section{§ 4. О булевозначном анализе}

В этом параграфе мы вкратце коснемся некоторых вопросов булевозначного анализа. Булевозначные расширения стандартных структур сильно отличаются по своим свойствам и структуре от нестандартных расширений. В частности, каноническое вложение $x \mapsto x^{\vee}$ стандартного универсума всех множеств $\mathbf{V}$ в булевозначный универсум $\mathbf{V}^{(\mathbf{B})}$ не является элементарным (кроме тривиальных случаев). Тем не менее, это отображение все же "переносит" в $\mathbf{V}^{(\mathbf{B})}$ аксиомы ZFC, что позволяет получать свойства объектов из стандартного универсума $\mathbf{V}$ путем рассмотрения существенно более простых объектов из булевозначного универсума $\mathbf{V}^{(\mathbf{B})}$. Мы дадим в п. 4е один пример такого такого рода редуцирования стандартных объектов к нестандартным.

4а. Булевозначные универсумы. Обычное понятие реляционной структуры $\mathfrak{A}=\left\langle A ; R_{1}, \ldots, R_{n}\right\rangle$, о котором шла речь в п. 1а, можно назвать 2 -значнъцм в том смысле, что выражение $R_{i}\left(a_{1}, \ldots, a_{m}\right)$ считается либо истинным, либо ложным, соответственно $\llbracket R_{i}\left(a_{1}, \ldots, a_{m}\right) \rrbracket=\top$ или $=\perp$, где $\llbracket \varphi \rrbracket$ обозначает оценку истинностного значения формулы $\varphi$.

Обобщая этот подход, рассматривают булевозначные структуры, т.е. такие, что соответствующие оценки принимают значения в какой-то фиксированной булевой алгебре В. Формально, каждое $R_{i}$ при этом становится функцией из $A^{r(i)}$ в $\mathbf{B}$, так что равенство $\llbracket R_{i}\left(a_{1}, \ldots, a_{r(i)}\right) \rrbracket=b$ (где $b \in \mathbf{B}$ ) означает, что оценка $R_{i}\left(a_{1}, \ldots, a_{r(i)}\right)=b$. Значения $b=\top_{\mathbf{B}}, \perp_{\mathbf{B}}$ (т.е. соответственно максимальный и минимальный элементы в $\mathbf{B}$ ) соответствуют обычной истинности и ложности. Оценки естественным образом распространяются с атомарных формул на более сложные. Например, $\varphi \wedge \psi \rrbracket=\llbracket \varphi \rrbracket \wedge \llbracket \psi \rrbracket, \llbracket \varphi \vee \psi \rrbracket=\llbracket \varphi \rrbracket \vee \llbracket \psi \rrbracket$, $\llbracket \neg \varphi \rrbracket=\complement \llbracket \varphi \rrbracket$, где в правых частях стоят операции $\wedge, \vee, \complement$ в булевой алгебре $\mathbf{B}$. Индуктивное определение оценки для кванторов, т.е. $\llbracket \exists x(x) \rrbracket=\bigvee_{x \in A} \llbracket \varphi(x) \rrbracket$ и аналогично для $\forall$, требует, конечно, полноты рассматриваемой алгебры $\mathbf{B}$, которая обычно предполагается; множество $A$ должно быть отдельно указано.

Мы уже видели во введении, как $\mathscr{P}(I)$-значная оценка естественным образом возникает при определении ультрастепени $\mathfrak{A}^{I} / U$. Другой общий способ построения булевозначных структур ведет к булевозначному универсуму $\mathbf{V}^{(\mathbf{B})}$, где $\mathbf{B}$ - фиксированная полная булева алгебра. Сам универсум $\mathbf{V}^{(\mathbf{B})}$ определяется как наименьший класс такой, что любая функция из некоторого множества $X \subseteq \mathbf{V}^{(\mathbf{B})}$ в $\mathbf{B}$ снова принадлежит $\mathbf{V}^{(\mathbf{B})}$. Таким образом, $x \in \mathbf{V}^{(\mathbf{B})}$, когда $x$ - функция, $\operatorname{dom} x \subseteq \mathbf{V}^{(\mathbf{B})}$ и $\operatorname{ran} x \subseteq \mathbf{B}$. Следующее определение вводит оценки атомарных формул с параметрами из $\mathbf{V}^{(\mathbf{B})}$ :

$$
\begin{aligned}
& \llbracket x \in y \rrbracket_{\mathbf{B}}=\bigvee_{z \in \operatorname{dom} y}\left(y(z) \cdot \llbracket z=x \rrbracket_{\mathbf{B}}\right), \\
& \llbracket x=y \rrbracket_{\mathbf{B}}=\bigwedge_{z \in \operatorname{dom} x}\left(x(z) \rightarrow \llbracket z \in y \rrbracket_{\mathbf{B}}\right) \cdot \bigwedge_{z \in \operatorname{dom} y}\left(y(z) \rightarrow \llbracket z \in x \rrbracket_{\mathbf{B}}\right),
\end{aligned}
$$

где $x, y \in \mathbf{V}^{(\mathbf{B})}$, а $p \rightarrow q$ определяется как $(\mathcal{C} p) \vee q$ в алгебре В. Как видно, ключевая идея этого определения (которое проходит индукцией по фоннеймановскому рангу $\mathrm{rk}$ множеств $\left.x, y \in \mathbf{V}^{(\mathbf{B})}\right)$ состоит в том, что если $x \in$ 
$\operatorname{dom} y$, то $\llbracket x \in y \rrbracket \geqslant y(x)$. Оценка $\llbracket \varphi \rrbracket_{\mathbf{B}}$ распространяется на более сложные формулы так, как указано выше.

Определим $x \approx_{\mathbf{B}} y$, если $\llbracket x=y \rrbracket_{\mathbf{B}}=\top_{\mathbf{B}}$. Вообще говоря, $x \approx_{\mathbf{B}} y$ не влечет $x=y$, однако, используя метод замечания 1.6 , построение $\mathbf{V}^{(\mathbf{B})}$ можно реорганизовать так, чтобы импликация $\left(x \approx_{\mathbf{B}} y\right) \Rightarrow(x=y)$ имела место, см. [50].

Наиболее известные приложения булевозначного универсума $\mathbf{V}^{(\mathbf{B})}$ связаны с доказательствами независимости разных математических предложений от ZFC. Например, чтобы доказать, что некоторое предложение $\varphi$ не зависит от аксиом ZFC, достаточно найти булевы алгебры $\mathbf{B}_{1}$ и $\mathbf{B}_{2}$ такие, что $\llbracket \varphi \rrbracket_{\mathbf{B}_{1}}=\top_{\mathbf{B}_{1}}$ и $\llbracket \neg \rrbracket_{\mathbf{B}_{2}}=\top_{\mathbf{B}_{2}}$. Такой подход, известный как форсинг, основан на пункте (i) следующей принципиальной теоремы.

ТЕОРемА 4.1. Пусть В - полная булева алгебра.

(i) Булевозначный универсум $\mathbf{V}^{(\mathbf{B})}$ удовлетворяет теории ZFC в том смысле, что $\llbracket \varphi \rrbracket_{\mathbf{B}}=\top_{\mathbf{B}}$ для любой логической и специальной аксиомь и любой теоремы ZFC.

(ii) ("достижимость") Ecли $x_{1}, \ldots, x_{n} \in \mathbf{V}^{(\mathbf{B})} u \llbracket \exists y \varphi\left(x_{1}, \ldots, x_{n}, y\right) \rrbracket_{\mathbf{B}}=b \in$ $\mathbf{B}$, то найдется элемент $y \in \mathbf{V}^{(\mathbf{B})}$, для которого $\llbracket \varphi\left(x_{1}, \ldots, x_{n}, y\right) \rrbracket_{\mathbf{B}} \geqslant b$.

Теорема сохраняет силу и для более широкого понятия гейтинговой алгебры с той поправкой, что тогда закон исключенного третьего в $\mathbf{V}^{(\mathbf{B})}$ не обязательно выполнен с оценкой Т В $_{\text {. }}$

Однако собственно булевозначный анализ (т.е. исключая форсинг) занимается исследованием тех свойств, которые объекты из $\mathbf{V}^{(\mathbf{B})}$ имеют с оценкой $\top_{\mathbf{B}}$, и возникающих отсюда свойств структур из универсума V. Ниже мы приведем несколько примеров, по необходимости опуская детали.

4b. Вложения стандартного универсума в булевозначный. Легко определить каноническое вложение $x \mapsto x^{\vee}$ всего универсума $\mathbf{V}$ в $\mathbf{V}^{(\mathbf{B})}$ : положим $\varnothing^{\vee}=\varnothing$ и далее $y^{\vee}\left(x^{\vee}\right)=\top_{\mathbf{B}}$, если $x \in y$. Получаем

$$
x \in y \Leftrightarrow \llbracket x^{\vee} \in y^{\vee} \rrbracket_{\mathbf{B}}=\top_{\mathbf{B}} \quad \text { и } \quad x \neq y \Leftrightarrow \llbracket x^{\vee}=y^{\vee} \rrbracket_{\mathbf{B}}=\perp_{\mathbf{B}},
$$

и в этом смысле получаем $\in$-вложение, относительно которого булевозначный универсум $\mathbf{V}^{(\mathbf{B})}$ является расширением $\mathbf{V}$. Это вложение имеет другие свойства, по сравнению с вложением $x \mapsto{ }^{*} x$ в нестандартном анализе. Например,

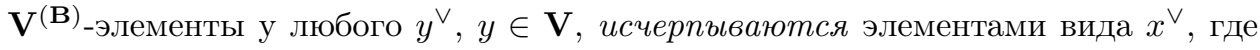
$x \in y$, в том смысле, что

$$
\llbracket z \in y^{\vee} \rrbracket_{\mathbf{B}}=\bigvee_{x \in y} \llbracket z=x^{\vee} \rrbracket_{\mathbf{B}} .
$$

Это, конечно, не имеет места для *-вложений вследствие аксиом типа Идеализации.

Вложение $x \mapsto x^{\vee}$ не является, вообще говоря, элементарным, и универсумы $\mathbf{V}$ и $\mathbf{V}^{(\mathbf{B})}$ не элементарно эквивалентны как $\in$-структуры. С другой стороны, нетрудно проверить, что $\llbracket \mathbb{N}^{\vee}-$ ряд всех натуральных чисел $\rrbracket_{\mathbf{B}}=\top_{\mathbf{B}}$, $\llbracket \mathbb{Q}^{\vee}$ - поле всех рациональных чисел $\rrbracket_{\mathbf{B}}=\top_{\mathbf{B}}$ и т. д. В то же время, обычно

$\llbracket \mathbb{R}^{\vee}$ - собственное плотное подполе поля всех вещественных чисел $\rrbracket_{\mathbf{B}}=\top_{\mathbf{B}}$ 
Из теоремы 4.1(i) следует: если $\exists x \varphi(x)$ - теорема ZFC, то существует элемент $x \in \mathbf{V}^{(\mathbf{B})}$, для которого $\llbracket \varphi(x) \rrbracket_{\mathbf{B}}=\boldsymbol{\top}_{\mathbf{B}}$. На этом основано следующее удобное определение. Если множество $X \in \mathbf{V}$ однозначно определяется формулой $\varphi(\cdot)$ в том смысле, что $X$ - единственное множество, удовлетворяющее $\varphi(X)$ в $\mathbf{V}$, и, сверх того, $\exists$ ! $X \varphi(X)$ - теорема $\mathbf{Z F C}$, то обозначим $X^{(\mathbf{B})}$ единственный с точностью до отношения $\approx_{\mathbf{B}}$ элемент $X^{\prime} \in \mathbf{V}^{(\mathbf{B})}$, для которого $\llbracket \varphi\left(X^{\prime}\right) \rrbracket_{\mathbf{B}}=\top_{\mathbf{B}}$. (Предполагается, что из всех формул $\varphi$, определяющих $X$, как-то выбрана одна формула.)

Теперь можно определить, например, $n^{(\mathbf{B})}$ для любого натурального числа $n$, $\mathbb{N}^{(\mathbf{B})}, \mathbb{R}^{(\mathbf{B})}$ и т. д., так что, скажем, $\llbracket n^{(\mathbf{B})}=n^{\vee} \rrbracket_{\mathbf{B}}=\top_{\mathbf{B}}, \llbracket \mathbb{N}^{(\mathbf{B})}=\mathbb{N}^{\vee} \rrbracket_{\mathbf{B}}=\top_{\mathbf{B}}$, $\llbracket \mathbb{Q}^{(\mathbf{B})}=\mathbb{Q}^{\vee} \rrbracket_{\mathbf{B}}=\top_{\mathbf{B}}$ и т. д. Но $\llbracket \mathbb{R}^{\vee} \varsubsetneqq \mathbb{R}^{(\mathbf{B})} \rrbracket_{\mathbf{B}}=\top_{\mathbf{B}}$. В определении $X^{(\mathbf{B})}$ речь не идет об определении $X^{(\mathbf{B})}$ для каждого $X \in \mathbf{V}$. Однако для важных математических объектов $X$ такое определение $X^{(\mathbf{B})}$ возможно.

В частности, это относится к полным метрическим пространствам в следующем смысле. Предположим, что $\mathbb{X}$ - такое пространство. Нетрудно проверить, что тогда

$\llbracket \mathbb{X}^{\vee}$ - метрическое (не обязательно полное) пространство $\rrbracket_{\mathbf{B}}=\top_{\mathbf{B}} \cdot{ }^{32}$

Следовательно, согласно теореме 4.1(ii), найдется элемент $\mathbb{X}^{\sim} \in \mathbf{V}^{(\mathbf{B})}$, удовлетворяющий равенству $\llbracket \mathbb{X}^{\sim}$ есть пополнение $\mathbb{X}^{\vee} \rrbracket_{\mathbf{B}}=\top_{\mathbf{B}} \cdot{ }^{33}$ С точки зрения обсуждаемых ниже вопросов, $\mathbb{X}^{\sim}-$ как бы "то же самое пространство $\mathbb{X}$, рассмотренное внутри $\mathbf{V}^{(\mathbf{B})}$ ".

После этого можно определить $F^{\sim}$ для всякого замкнутого $F \subseteq \mathbb{X}$ как замыкание $F^{\vee}$ в $\mathbb{X}^{\sim}$, и далее $\mathscr{O}^{\sim}$ для всякого открытого $\mathscr{O} \subseteq \mathbb{X}$ через двукратный переход к дополнениям и, наконец, $X^{\sim}$ для всякого борелевского множества $X \subseteq \mathbb{X}$ трансфинитной индукцией по построению $X$ из открытых множеств при помощи операций дополнения и счетного объединения.

4c. Теоремы переноса для булевозначного универсума. Как отмечалось, каноническое вложение $x \mapsto x^{\vee}$ не является, вообще говоря, элементарным. Вследствие этого схема переноса для булевозначных универсумов имеет более сложный и менее универсальный характер, чем для нестандартных универсумов. С другой стороны, перенос дает здесь математически значимые результаты. В первом приближении, схема переноса состоит здесь в следующем.

${ }^{32}$ Строго говоря, $\mathbb{X}$ есть пара, состоящая из собственно множества $\mathbb{X}$ и функции расстояния $d: \mathbb{X}^{2} \rightarrow \mathbb{R}_{+}$, отождествляемой, как обычно, с множеством $d=\{\langle\langle x, y\rangle, d(x, y)\rangle: x, y \in \mathbb{X}\}$. Соответственно, $\mathbb{X}^{\vee}$ состоит из $\mathbb{X}^{\vee}$ и $d^{\vee}$. Естественно, если $\mathbb{X}$ несет какую-то дополнительную структуру, скажем, групповые или кольцевые операции, то еe ${ }^{\vee}$-образ также присоединяется к $\mathbb{X}^{\vee}$, и эти операции по возможности продолжаются на $X^{\sim}$.

${ }^{33} \mathrm{~B}$ продолжение сказанного в сноске 32 , заметим, что если $\mathbb{X}$ имеет дополнительную структуру топологического типа, то можно ставить вопрос о ее продолжении с $\mathbb{X}^{\vee}$ на $\mathbb{X}^{\sim}$ в $\mathbf{V}^{(\mathbf{B})}$. Например, если +- непрерывная групповая операция на $\mathbb{X}$, то $+^{\vee}$ по простым соображениям остается непрерывной групповой операцией на $\mathbb{X}^{\vee}$ с оценкой $\top_{\text {В. }}$ Если $\mathbb{X}-$ польское, т.е. сепарабельное полное метрическое пространство, то рассуждение, основанное на теореме абсолютности Шенфилда, позволяет получить элемент $+\sim \in \mathbf{V}^{(\mathbf{B})}$, который с оцен-

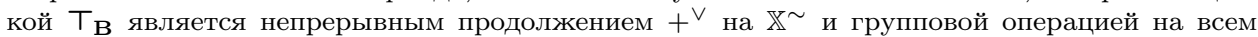
пространстве $\mathbb{X}^{\sim}$, а последнее остается польским в $\mathbf{V}^{(\mathbf{B})}$ с оценкой $\top_{\mathbf{B}}$. Естественно, в этом случае продолженная операция $+^{\sim}$ присоединяется к структуре $\mathbb{X} \sim$, превращая его в польскую группу в $\mathbf{V}^{(\mathbf{B})}$ с оценкой $\boldsymbol{T}_{\mathbf{B}}$. 
(I) Поиск нестандартного представления исходной математической структуры $\mathfrak{A}$ в V. Под этим понимается некоторая математически более простая структура $\mathbb{X}$ в универсуме $\mathbf{V}$ (например, метрическое пространство) такая, что множество $\left(\mathbb{X}^{\sim}\right)^{\wedge}=\left\{r \in \mathbf{V}^{(\mathbf{B})}: \llbracket r \in \mathbb{X}^{\sim} \rrbracket_{\mathbf{B}}=\top\right\}$ всех "до-

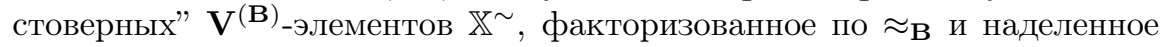
подходящей структурой, изоморфно $\mathfrak{A}$. Без факторизации, семейство $\left(\mathbb{X}^{\sim}\right)^{\wedge}$ является, вообще говоря, собственным классом. После факторизации, оно становится множеством, или, точнее говоря, превращается в множество после урезания классов эквивалентности по методу из замечания 1.6.

(II) Исходная структура $\mathfrak{A}$ становится предметом изучения. Например, мы хотим доказать, что некоторое предложение $\Phi$ истинно в $\mathfrak{A}$. Достаточно проверить, что $\Phi$ истинно в изоморфной модели $\left(\mathbb{X}^{\sim}\right)^{\wedge}$. Для этого подбирается другая формула $\varphi$, связанная с $\Phi$ так, что из истинности $\varphi$ в $\mathbb{X}^{\sim}$ с оценкой $\top_{\text {в в }} \mathbf{V}^{(\mathbf{B})}$ следует истинность $\Phi$ в $\left(\mathbb{X}^{\sim}\right)^{\wedge},-$ именно в этом

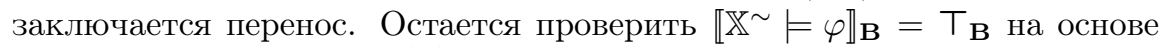
свойств объекта $X^{\sim}$ в $\mathbf{V}^{(\mathbf{B})}$ и содержания $\varphi$.

Итак, первая задача здесь состоит в подборе структуры $\mathbb{X}$ такой, что $\mathfrak{A}$ изоморфна $\left(\mathbb{X}^{\sim}\right)^{\wedge}$ (при подходящем выборе булевой алгебры В, часто последняя заранее связана с $\mathfrak{A}$ ). Ниоткуда не следует, что такой выбор $\mathbb{X}$ по $\mathfrak{A}$ возможен в каждом случае, однако если такая возможность находится, то техника переноса в духе (II), детали которой также индивидуальны, может значительно облегчить исследование исходной структуры $\mathfrak{A}$.

Первый существенный пример такого рода был дан Е. И. Гордоном [57], показавшим, что известные в функциональном анализе K-пространства изоморфны структурам вида $\left(\mathbb{R}^{\sim}\right)^{\wedge}$ при выборе в качестве В булевой алгебры всех идемпотентных элементов $\left(x^{2}=x\right)$ исходного K-пространства. Ниже мы укажем еще один пример из [58], который приводится здесь в частном случае и с другим, логического характера, доказательством.

4d. Нестандартные представления полных метрических пространств. Этот пункт является подготовительным к изложению упомянутого примера. Чтобы не повторяться, фиксируем полную булеву алгебру $\mathbf{B}$ и условимся опускать индекс в в обозначениях $\llbracket \cdot \rrbracket_{\mathbf{B}}, \mathbf{T}_{\mathbf{B}}, \perp_{\mathbf{B}}, \approx_{\mathbf{B}}$.

Фиксируем также некоторое полное метрическое пространство $\mathbb{X}$; тем самым определены элементы $\mathbb{X}^{\vee}, \mathbb{X}^{\sim} \in \mathbf{V}^{(\mathbf{B})}$ и совокупность $\left(\mathbb{X}^{\sim}\right)^{\wedge}$ всех “достоверных элементов" из $\mathbb{X} \sim$, см. п. 4b. Нашей целью будет построение такого отображения из $\left(\mathbb{X}^{\sim}\right)^{\wedge}$ в множество $\mathbf{C}^{\prime \prime}(\mathscr{S}, \mathbb{X})$ всех функций со значениями в $\mathbb{X}$, определенных и непрерывных на плотных $\mathbf{G}_{\delta}$-множествах стоунова пространства $\mathscr{S}$ алгебры $\mathbf{B}$, которое окажется биекцией с точностью до эквивалентности $\approx$ на $\left(\mathbb{X}^{\sim}\right)^{\wedge}$ и следующей эквивалентности на $\mathbf{C}^{\prime \prime}(\mathscr{S}, \mathbb{X})$ :

$$
f \equiv g, \quad \text { если множество }\{s \in \operatorname{dom} f \cap \operatorname{dom} g: f(s)=g(s)\} \text { плотно в } \mathscr{S} \text {. }
$$

Напомним, что стоуново пространство $\mathscr{S}=\mathscr{S}^{\mathbf{B}}$ булевой алгебры В образовано всеми ультрафильтрами $s \subseteq \mathbf{B}$ с топологией, порожденной множествами вида $\mathscr{S}_{b}=\{s \in \mathscr{S}: b \in s\}$, где $b \in \mathbf{B}$. Стоуновы пространства полных булевых алгебр обладают рядом специальных свойств, в частности, в них замыкания 
открытых множеств открыты. Хорошо известно, что булева алгебра В каноническим образом отождествляется с семейством всех открыто-замкнутых подмножеств пространства $\mathscr{S}$ посредством отображения $b \mapsto \mathscr{S}_{b} \cdot{ }^{34}$ При этом операции $\wedge, \vee, \mathcal{C}$ в алгебре $\mathbf{B}$ соответствуют теоретико-множественным операциям пересечения, объединения и дополнения на открыто-замкнутых множествах в $\mathscr{S}^{35}$ Например, $\mathscr{S}_{b \vee c}=\mathscr{S}_{b} \cup \mathscr{S}_{c}, \mathscr{S}_{\mathrm{C} b}=\mathscr{S} \backslash \mathscr{S}_{b}$. Также очевидно, что $\mathscr{S}_{\top}=\mathscr{S}_{\text {и }} \mathscr{S}_{\perp}=\varnothing$.

Через $\mathscr{F}$ обозначим семейство всех непустых замкнутых множеств $F \subseteq \mathbb{X}$.

Tеорема 4.2. Пусть В, $\mathbb{X}, \mathscr{S}$ таковы, как указано выше.

(i) Каждому $p \in\left(\mathbb{X}^{\sim}\right)^{\wedge}$ можно сопоставить функиию $\mathbf{f}_{p} \in \mathbf{C}^{\prime \prime}(\mathscr{S}, \mathbb{X})$ так, что $p \approx q \Rightarrow \mathbf{f}_{p}=\mathbf{f}_{q} u$, кроме того, для любых $b \in \mathbf{B} u F \in \mathscr{F}$ выполнено:

$$
\llbracket p \in F^{\sim} \rrbracket \geqslant b \Leftrightarrow \mathbf{f}_{p} "\left(\mathscr{S}_{b} \cap \operatorname{dom} \mathbf{f}_{\xi}\right) \subseteq F .
$$

(ii) Каждой функиии $f \in \mathbf{C}^{\prime \prime}(\mathscr{S}, \mathbb{X})$ можно сопоставить элемент $p=\mathbf{p}_{f} \in$ $\left(\mathbb{X}^{\sim}\right)^{\wedge}$ maк, что $f \equiv \mathbf{f}_{p} u$, кроме того, $f \equiv g \Rightarrow \mathbf{p}_{f} \approx \mathbf{p}_{g}$.

Это означает, что отображения $p \mapsto \mathbf{f}_{p} u f \mapsto \mathbf{p}_{f}$ индуцируют биекцию из $\left(\mathbb{X}^{\sim}\right)^{\wedge} / \approx$ на $\mathbf{C}^{\prime \prime}(\mathscr{S}, \mathbb{X}) / \equiv$ и обратную $к$ ней биекиию из $\mathbf{C}^{\prime \prime}(\mathscr{S}, \mathbb{X}) / \equiv н а$ $\left(\mathbb{X}^{\sim}\right)^{\wedge} / \approx$

ДокАЗАтельство. (i) Положим $\mathscr{F}_{k}=\left\{F \in \mathscr{F}:\right.$ диаметр $\left.F \leqslant k^{-1}\right\}$. Если $F \in \mathscr{F}_{k}$, то по простым соображениям $F^{\sim}$ является замкнутым множеством диаметра $\leqslant k^{-1}$ в $\mathbb{X}^{\sim}$ в $\mathbf{V}^{(\mathbf{B})}$ с оценкой $\top$. Каждое множество $G_{k}=$ $\bigvee_{F \in \mathscr{F}_{k}} \mathscr{S}_{\llbracket p \in F \sim \rrbracket}$ плотно в пространстве $\mathscr{S}$. В самом деле, рассмотрим произвольное $b \in \mathbf{B}, b \neq \perp$. Напомним, что $F^{\vee}$ плотно в $\mathbb{X}^{\sim}$ в $\mathbf{V}^{(\mathbf{B})}$ с оценкой $\boldsymbol{\top}$, а потому найдутся $c \in \mathbf{B}, c \leqslant b$, и точка $x \in \mathbb{X}$ такие, что, с оценкой $\geqslant c$ в $\mathbf{V}^{(\mathbf{B})}$, расстояние между $x^{\vee}$ и $p$ в $\mathbb{X}^{\sim}$ не превосходит $(2 k)^{-1}$. Но это означает, что $\llbracket \xi \in F^{\sim} \rrbracket \geqslant c$, где $F \in \mathscr{F}_{k}-$ замкнутый шар диаметра $k^{-1}$ с центром в $x$. Тем самым, $\mathscr{S}_{c} \subseteq G_{k}$, что и требовалось.

Итак, $S_{p}=\bigcap_{k} G_{k}$ есть плотное $\mathbf{G}_{\delta}$-множество в $\mathscr{S}$.

По определению, если $s \in S_{p}$, то множество $\mathscr{F}_{p}(s)=\left\{F \in \mathscr{F}: \llbracket p \in F^{\sim} \rrbracket \in s\right\}$ непусто пересекается с каждым $\mathscr{F}_{k}$. С другой стороны, если $R$ и $Q$ - дизъюнктные замкнутые множества в $\mathbb{X}$, то нетрудно вывести $\llbracket R^{\sim} \cap Q^{\sim} \neq \varnothing \rrbracket=\perp$, а потому оценки $\llbracket p \in R^{\sim} \rrbracket$ и $\llbracket p \in Q^{\sim} \rrbracket$ несовместимы в $\mathbf{B}$ и, следовательно, не могут обе вместе принадлежать ультрафильтру $s$. Это означает, что $\mathscr{F}_{p}(s)$ является фильтром Коши (при $s \in S_{p}$ ), так что пересечение $\bigcap \mathscr{F}_{p}(s)$ содержит единственную точку, которую мы обозначим через $\mathbf{f}_{p}(s)$. Этим определена непрерывная функция $\mathbf{f}_{p}: S_{p} \rightarrow \mathbb{X}$.

Остается доказать (16) для любых $b \in \mathbf{B}$ и $F \in \mathscr{F}$. Допустим, что $\llbracket p \in F^{\sim} \rrbracket$ $b$. Тогда $b^{\prime}=\llbracket p \notin F^{\sim} \rrbracket \wedge b \neq \perp$. По определению операции $\sim^{\sim}$ отсюда следует, что найдутся точка $x \in \mathbb{X}$ и число $k$ такие, что 1$)$ расстояние от $x$ до $F$ в $\mathbb{X}$ больше $\frac{2}{k}$ и 2) $b^{\prime \prime}=b^{\prime} \wedge \llbracket$ расстояние от $x^{\vee}$ до $p$ в $\mathbb{X} \sim<\frac{1}{k} \rrbracket \neq \perp$. Далее, по

\footnotetext{
${ }^{34}$ Каждое $\mathscr{S}_{b}$ открыто по определению и замкнуто, поскольку множество $\mathscr{S} \backslash \mathscr{S}_{b}=\mathscr{S}_{b}$ также открыто. Если $X \subseteq \mathscr{S}$ открыто-замкнуто, то найдутся множества $A, B \subseteq \mathbf{B}$ такие, что $X=\bigcup_{b \in A} \mathscr{S}_{b}$ и $\mathscr{S} \backslash X=\bigcup_{b \in B} \mathscr{S}_{b}$. Нетрудно проверить, что $X=\mathscr{S}_{a}$, где $a=\bigwedge A$ в $\mathbf{B}$.

35 Это свойство не сохраняется для бесконечных операций: например, бесконечной операции $\bigvee A$ в В соответствует, в $\mathscr{S}$, операция "замыкание $\bigcup_{a \in A} \mathscr{S}_{a}$ ", а не само объединение $\bigcup_{a \in A} \mathscr{S}_{a}$.
} 
построению, найдется множество $F_{1} \in \mathscr{F}_{k}$ такое, что $c=b^{\prime \prime} \wedge \llbracket p \in F_{1} \sim \rrbracket \neq \perp$. Понятно, что $F_{1}$ включено в $\frac{2}{k}$-окрестность точки $x$, а потому $F_{1} \cap F=\varnothing$. Однако, снова по построению, мы имеем $\mathbf{f}_{p}(s) \in F_{1}$ для всех $s \in \mathscr{S}_{c} \cap S_{p}$, а потому правая часть эквивалентности леммы не может иметь места (ведь $\left.\varnothing \neq \mathscr{S}_{c} \cap S_{p} \subseteq \mathscr{S}_{b}\right)$.

Обратно, допустим, что $\mathbf{f}_{p}(s) \notin F$ хотя бы для одного $s \in \mathscr{S}_{b} \cap S_{p}$. Согласно непрерывности $\mathbf{f}_{p}$ и замкнутости $F$, найдутся $b^{\prime} \in \mathbf{B}, x \in \mathbb{X}$ и число $k$ такие, что $\perp \neq b^{\prime} \leqslant b$, расстояние от $x$ до $F$ в $\mathbb{X}$ больше $\frac{2}{k}$ и $\mathbf{f}_{p}(s)$ принадлежит $\frac{1}{k}$-окрестности точки $x$ при любом $s \in \mathscr{S}_{b^{\prime}} \cap S_{p}$. Снова берем множество $F_{1} \in \mathscr{F}_{k}$ такое, что $c=b^{\prime} \wedge \llbracket p \in F_{1} \sim \rrbracket \neq \perp$. По построению, $\mathbf{f}_{p}(s) \in F_{1}$ для всех $s \in$ $\mathscr{S}_{c} \cap G_{p}$, а потому $F_{1} \cap F=\varnothing$. Значит, $\llbracket p \notin F^{\sim} \rrbracket \geqslant c$, а потому $\llbracket p \notin F^{\sim} \rrbracket \ngtr b$.

(ii) Пусть $G=\operatorname{dom} f=\bigcap_{k} G_{k}$, где каждое $G_{k}$ является плотным открытым подмножеством $\mathscr{S}$. Мы выполним, в сущности, эквивалентное построение фильтра стягивающихся замкнутых множеств. Именно, определим элемент $C_{f} \in \mathbf{V}^{(\mathbf{B})}$ так:

$$
C_{f}\left(R^{\sim}\right)=\bigvee B_{f}(R), \quad \text { где } \quad B_{f}(R)=\left\{b \in \mathbf{B}: f^{\prime \prime}\left(\mathscr{S}_{b} \cap \operatorname{dom} f\right) \subseteq R\right\}
$$

для любого множества $R \in \mathscr{F}$. Утверждается, что $\Phi \rrbracket=\top$, где $\Phi$ обозначает формулу " $C_{f}$ состоит из замкнутых подмножеств $\mathbb{X} \sim$, замкнуто относительно операции $\cap$ и, для всякого $k \in \mathbb{N}^{\vee}, C_{f}$ содержит множество $F$ диаметра $\leqslant k^{-1}$ ".

В самом деле, предположим, что, например, $\llbracket C_{f}$ не $\cap$-замкнуто $=b>\perp$. Существуют множества $R, Q \in \mathscr{F}$ такие, что $\llbracket R^{\sim}, Q^{\sim} \in C_{f} \wedge F^{\sim} \notin C_{f} \rrbracket=b^{\prime}>\perp$, где $F=R \cap Q$. По определению, $b \leqslant \bigvee B_{f}(R)$ и $b \leqslant \bigvee B_{f}(Q)$, так что найдутся элементы $a_{1} \in B_{f}(R)$ и $a_{2} \in B_{f}(Q)$, удовлетворяющие $b^{\prime \prime}=b^{\prime} \wedge a_{1} \wedge$ $a_{2}>\perp$. Однако, снова по определению, $a_{3}=a_{1} \wedge a_{2}$ принадлежит $B_{f}(F)$, откуда $\llbracket F^{\sim} \in C_{f} \rrbracket \geqslant a_{3}$. Наконец, $a_{1}$ и $b$ совместны в $\mathbf{B}$, так как $b \wedge a_{3} \geqslant b^{\prime \prime}>\perp-$ противоречие.

Из соотношения $\llbracket \Phi \rrbracket=\top$ следует, что найдется элемент $p \in \mathbf{V}^{(\mathbf{B})}$, удовлетворяющий $\llbracket p \in \mathbb{X} \sim \rrbracket=\top$ и $\llbracket R^{\sim} \in C_{f} \Rightarrow p \in R^{\sim} \rrbracket=\top$ для любого $R \in \mathscr{F}$. Отсюда следует $\llbracket p \in R^{\sim} \rrbracket=\bigvee B_{f}(R)=C_{f}\left(R^{\sim}\right)$ в алгебре $\mathbf{B}$ для каждого $R \in \mathscr{F}$.

Теперь следует доказать, что $\mathbf{f}_{p} \equiv f$. Мы утверждаем, что $f$ и $\mathbf{f}_{p}$ совпадают на пересечении $G \cap S_{p}$ плотных $\mathbf{G}_{\delta}$ множеств $G=\operatorname{dom} f$ и $S_{p}=\operatorname{dom} \mathbf{f}_{p}$. Предположим противное. Тогда найдется $b \in \mathbf{B}, b \neq \perp$, и пара дизъюнктных множеств $R, Q \in \mathscr{F}$ такие, что $\mathbf{f}_{p} "\left(\mathscr{S}_{b} \cap S_{p}\right) \subseteq R$ и $f^{\prime \prime}\left(\mathscr{S}_{b} \cap G\right) \subseteq Q$. Тогда $\llbracket p \in R^{\sim} \rrbracket \geqslant b$ по (16). С другой стороны, по определению, $b \in B_{f}(Q)$, а потому $b \leqslant C_{f}\left(Q^{\sim}\right)$ в $\mathbf{B}$, откуда $\llbracket p \in Q^{\sim} \rrbracket \geqslant \llbracket Q^{\sim} \in C_{f} \rrbracket \geqslant b$. Итак, $\llbracket R^{\sim} \cap Q^{\sim} \neq \varnothing \rrbracket \geqslant b>\perp$, противоречие.

Наконец, вследствие непрерывности наших функций, $f \equiv g$ влечет $B_{f}(R)=$ $B_{g}(R)$ для любого $R \in \mathscr{F}$, так что из $f \equiv g$ следует $\mathbf{p}_{f} \approx \mathbf{p}_{g}$. Теорема 4.2 доказана.

В некоторых случаях теорема 4.2 может быть уточнена в части выбора класса функции $f$, представляющей элемент $p \in\left(\mathbb{X}^{\sim}\right)^{\wedge}$.

1) Например, предположим, что $\mathbb{X}$ - компактное (метрическое) пространство. Тогда для любого $k$ найдется конечное покрытие $\mathbb{X}=R_{1}^{k} \cup \cdots \cup R_{n(k)}^{k}$ множествами $R_{i} \in \mathscr{F}$ диаметра $\leqslant k^{-1}$. Тогда $\top=\llbracket p \in \mathbb{X} \sim \rrbracket=\llbracket p \in R_{1}^{k \sim} \rrbracket \vee \cdots \vee$ $\llbracket p \in R_{n(k)}^{k} \stackrel{\sim}{\rrbracket}$, а потому, какова бы ни была точка $s \in \mathscr{S}$, имеем $\llbracket p \in R_{i}^{k} \rrbracket \in s$ 
для подходящего $1 \leqslant i \leqslant n(k)$. Получаем ситуацию, рассмотренную выше (доказательство (i)), с тем дополнением, что $G_{k}=\mathscr{S}$ для всех $k$, т.е. $S_{p}=\mathscr{S}$. Таким образом, в этом случае теорема 4.2 может быть усилена посредством замены $\mathbf{C}^{\prime \prime}(\mathscr{S}, \mathbb{X})$ на (много меньшее) множество $\mathbf{C}(\mathscr{S}, \mathbb{X})$ всех всюду определенных непрерывных функций $\mathscr{S} \rightarrow \mathbb{X}$.

2) Предположим теперь, что пространство $\mathbb{X}$ является только $\boldsymbol{\sigma}$-компактным, т.е. $\mathbb{X}=\bigcup_{k} F_{k}$, где каждое $F_{k}$ компактно. Можно считать, что каждое $F_{k}$ предсталяет собой замкнутый шар $F_{k}=\left\{x \in \mathbb{X}: \rho\left(x, x_{k}\right) \leqslant \varepsilon_{k}\right\}$, где $x_{k} \in \mathbb{X}$ и $\varepsilon_{k}>0$. Пусть теперь $p \in\left(\mathbb{X}^{\sim}\right)^{\wedge}$. Можно показать ${ }^{36}$, что $\bigvee_{k} \llbracket p \in F_{k} \sim=\top$,

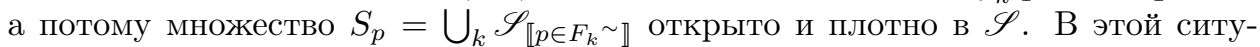
ации непрерывная функция $\mathbf{f}_{p}: S_{p} \rightarrow \mathbb{X}$ может быть определена отдельно на

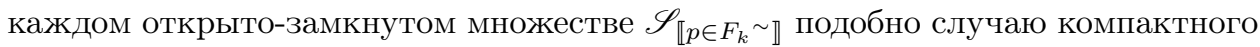
пространства. Итак, в $\sigma$-компактном случае теорема 4.2 может быть усилена заменой $\mathbf{C}^{\prime \prime}(\mathscr{S}, \mathbb{X})$ на множество $\mathbf{C}^{\prime}(\mathscr{S}, \mathbb{X})$ функций, определенных и непрерывных на плотных открытых подмножествах $\mathscr{S}$.

3) В случае локально компактного, но не сепарабельного пространства $\mathbb{X}$ возникают проблемы с тем рассуждением, о котором сказано в сноске 36 . Однако эта трудность может быть легко обойдена в предположении, что локальная компактность $\mathbb{X}$ равномерна в том смысле, что для некоторого $r>0$ все шары радиуса $r$ в $\mathbb{X}$ компактны. Отметим, что это условие выполняется в случаях, когда пространство $\mathbb{X}$ несет подходящую алгебраическую структуру, например, структуру топологической группы. Локально компактные, но лишенные этого свойства равномерности пространства уводят в специальную область общей топологии.

4) Что касается неметрических пространств, то работы [31], [32], [59] содержат результаты, аналогичные теореме 4.2 и, отчасти, ее вариантам 1) и 2), но для равномерных пространств в смысле Бурбаки [60].

4е. Пример: кольца функций. В ситуации рассмотренной в п. 4d, отображение $p \mapsto \mathbf{f}_{p}$ может быть и алгебраическим изоморфизмом в тех случаях, когда пространство $\mathbb{X}$ является носителем какой-то алгебраической структуры.

В качестве примера рассмотрим случай, когда исходное полное метрическое пространство $\mathbb{X}$ является топологическим кольцом (обе операции непрерывны как функции двух аргументов). Для простоты считаем, ито $\mathbb{X}$ сепарабельно. Тогда операции $\mathbb{X}$ продолжаются в $\mathbf{V}^{(\mathbf{B})}$ на $\mathbb{X}^{\sim}$ (см. сноску 33 ), так что $\mathbb{X}^{\sim}$ остается топологическим кольцом в $\mathbf{V}^{(\mathbf{B})}$ с оценкой $\top$. Это позволяет придать кольцевую структуру фактор-множеству $\left(\mathbb{X}^{\sim}\right)^{\wedge} / \approx$ уже в исходном универсуме: $\left[\xi_{1}\right]_{\approx}+\left[\xi_{2}\right]_{\approx}=[\xi]_{\approx}$, где $\xi-$ единственный с точностью до $\approx$ элемент $\left(\mathbb{X}^{\sim}\right)^{\wedge}$, удовлетворяющий условию $\llbracket \xi_{1}+\xi_{2}=\xi \rrbracket=\top$ (корректность следует из теоремы 4.1(i)), и то же для умножения. С другой стороны, приняв покомпонентные операции, т.е., например, $(f+g)(x)=f(x)+g(x)$, превращаем фактормножество $\mathbf{C}^{\prime \prime}(\mathscr{S}, \mathbb{X}) / \equiv$ в кольцо.

ТЕОрема 4.3. В указанной ситуации отображение $p \mapsto \mathbf{f}_{p}$ является не только биекцией, но и изоморфизмом колеи $\left(\mathbb{X}^{\sim}\right)^{\wedge} / \approx u \mathbf{C}^{\prime \prime}(\mathscr{S}, \mathbb{X}) / \equiv$.

\footnotetext{
36Это не сразу очевидное рассуждение. Для вывода равенства $\llbracket \mathbb{X} \sim=\bigcup_{k \in \mathbb{N} \vee} F_{k} \sim=\top$, из которого следует $\bigvee_{k} \llbracket p \in F_{k} \sim \rrbracket=\top$, нужно записать оцениваемую формулу в виде $\Pi_{1}^{1}$ формулы, а затем воспользоваться теоремой абсолютности Шенфилда.
} 
Если пространство $\mathbb{X}$ компактно или, соответственно, локально компактно, то $\mathbf{C}^{\prime \prime}(\mathscr{S}, \mathbb{X})$ можно заменить здесъ на $\mathbf{C}(\mathscr{S}, \mathbb{X})$, соответственно, на $\mathbf{C}^{\prime}(\mathscr{S}, \mathbb{X})$.

ДокАЗАТЕЛьСтво. Проверим только сохранение аддитивной операции при указанном отображении. Предположим, что $p, q, r \in\left(\mathbb{X}^{\sim}\right)^{\wedge}$ и $\llbracket p=q+r \rrbracket=\top$. Требуется доказать, что соответствующие функции $\mathbf{f}_{p}, \mathbf{f}_{q}, \mathbf{f}_{r} \in \mathbf{C}^{\prime \prime}(\mathscr{S}, \mathbb{X})$ удовлетворяют условию $\mathbf{f}_{p}(s)=\mathbf{f}_{q}(s)+\mathbf{f}_{r}(s)$ (в кольце $\mathbb{X}$ ) для всех $s$ из плотного открытого множества $D=\operatorname{dom} \mathbf{f}_{p} \cap \operatorname{dom} \mathbf{f}_{q} \cap \operatorname{dom} \mathbf{f}_{r}$. Вследствие непрерывности этих функций и операции + , предположение противного дает нам два дизъюнктных множества $R, S \in \mathscr{F}$, еще множества $F, F^{\prime} \in \mathscr{F}$ и открыто-замкнутое множество вида $\mathscr{S}_{b}, b \in \mathbf{B}$, такие, что $\mathbf{f}_{p}(s) \in S, \mathbf{f}_{q}(s) \in F$ и $\mathbf{f}_{r}(s) \in F^{\prime}$ для всех $s \in D \cap \mathscr{S}_{p}$ и, кроме того, $x+y \in R$ для всех $x \in F$ и $y \in F^{\prime}$. Тогда элементы $c_{1}=\llbracket p \in S^{\sim} \rrbracket, c_{2}=\llbracket q \in F^{\sim} \rrbracket, c_{3}=\llbracket r \in F^{\prime \sim} \rrbracket$ булевой алгебры В принадлежат $s$. Но $s$ - ультрафильтр, а потому $d=c_{1} \wedge c_{2} \wedge c_{3}>\perp$. Итак, $\llbracket q \in F^{\sim} \wedge r \in F^{\prime \sim} \rrbracket \geqslant d>\perp$ в $\mathbf{B}$.

$\mathrm{C}$ другой стороны, нетрудно показать, что указанное соотношение между множествами $R, F, F^{\prime}$ абсолютно в том смысле, что $\forall x \in F^{\sim} \forall y \in F^{\prime \sim}(x+y \in$ $\left.R^{\sim}\right) \rrbracket=\top$. Отсюда следует $\llbracket p \in R^{\sim} \rrbracket \geqslant d>\perp$. Но это противоречит тому, что $\llbracket p \in S^{\sim} \rrbracket=c_{1} \geqslant d$, поскольку $\llbracket R^{\sim} \cap S^{\sim}=\varnothing \rrbracket=\top$. Теорема 4.3 доказана.

Доказанная теорема позволяет положить, например, $\mathbb{X}=\mathbb{C}=$ поле комплексных чисел ${ }^{37}$ и $\mathfrak{A}=$ кольцо $\mathbf{C}^{\prime}(\mathscr{S}, \mathbb{C}) / \equiv$ в схеме п. 4c. Далее, имеется класс формул, для которых перенос (II) в п. 4c возможен по чисто синтаксическим причинам. Напомним, что хорновой формулой называется всякая формула вида П $A$, где П - произвольная кванторная приставка, а $A$ - конъюнкция формул вида $\left(\alpha_{1} \wedge \alpha_{2} \wedge \cdots \wedge \alpha_{n}\right) \Rightarrow \alpha$ и $\alpha, \alpha_{k}$ - атомарные формулы данного языка.

Лемма 4.4. Пусть $\Phi$ - замкнутая хорнова формула языка теории колеи, с параметрами из $\left(\mathbb{C}^{\sim}\right)^{\wedge}$. Если $\llbracket \Phi$ истинна в $\mathbb{C}^{\sim} \rrbracket=\top$, то $\Phi$ истинна в $\left(\mathbb{C}^{\sim}\right)^{\wedge}$.

ДоказАтельство. Рассуждаем индукцией по сложности Ф. Индуктивные шаги для квантора $\forall$ и конъюнкции тривиальны. Индуктивный шаг для $\exists$ легко проверяется на основе теоремы 4.1(ii). Остается рассмотреть случай, когда $\Phi$ имеет вид $\left(\alpha_{1} \wedge \cdots \wedge \alpha_{n}\right) \Rightarrow \alpha$, где $\alpha$ и $\alpha_{k}$ - атомарные формулы языка теории колец. Предположим $\llbracket \mathbb{C}^{\sim} \models \Phi \rrbracket=\top$. Тогда $\llbracket \mathbb{C}^{\sim}=\alpha \rrbracket \geqslant \llbracket \mathbb{C}^{\sim} \models \alpha_{1} \wedge$ $\cdots \wedge \alpha_{n} \rrbracket$. Предполагая, что каждая $\alpha_{k}$ истинна в $\left(\mathbb{C}^{\sim}\right)^{\wedge}$, выведем то же для $\alpha$. Однако $\alpha_{k}$ - атомарная формула, например, $r+s=t$, где $r, s, t$ принадлежат $\left(\mathbb{C}^{\sim}\right)^{\wedge}$. По определению истинности в $\left(\mathbb{C}^{\sim}\right)^{\wedge}$, мы имеем $\llbracket \mathbb{C}^{\sim} \models \alpha_{k} \rrbracket=\top$, так что и $\llbracket \mathbb{C}^{\sim}=\alpha_{1} \wedge \cdots \wedge \alpha_{n} \rrbracket=\top$, откуда $\llbracket \mathbb{C}^{\sim} \models \alpha \rrbracket=\top$, т.е. атомарная формула $\alpha$ истинна в $\left(\mathbb{C}^{\sim}\right)^{\wedge}$, что и требовалось. Лемма 4.4 доказана.

СлЕДСтвиЕ 4.5. Предположим, что $\Phi$ - хорнова формула языка теории колеи, истинная в поле $\mathbb{C}$ комплексных чисел. Тогда в наших условиях $\Phi$ истинна в $\mathbf{C}^{\prime}(\mathscr{S}, \mathbb{C}) / \equiv$. В частности, в $\mathbf{C}^{\prime}(\mathscr{S}, \mathbb{C}) / \equiv$ любое алгебраическое уравнение степени $\geqslant 1$ имеет решение.

\footnotetext{
${ }^{37}$ Отметим важный факт: $\mathbb{C}^{\sim}$ - поле комплексных чисел в $\mathbf{V}^{(\mathbf{B})}$ в том смысле, что $\llbracket \mathbb{C}^{\sim}$ - поле комплексных чисел $=\top$. Аналогичное утверждение имеет место и для вещественных чисел $\mathbb{R}$. Для алгебры $\mathbf{C}^{\prime}(\mathscr{S}, \mathbb{R})$ и алгебраических уравнений нечетной степени легко доказать утверждение, аналогичное следствию 4.5 .
} 
Понятно, что хорновость играет ключевую роль в элиминации общего шага для дизъюнкции в доказательстве леммы 4.4. Мы не знаем достаточно общих результатов о таком переносе для нехорновых формул. Тем не менее, для некоторых примеров, указанных в [29], [59], “нехорнов” перенос оказывается возможным. Суть в том, что если в терминах доказательства леммы 4.4 $\llbracket \mathbb{C}^{\sim}=\alpha_{1} \vee \cdots \vee \alpha_{n} \rrbracket=\top$, то имеется "разложение единицы" $\top=b_{1} \vee \cdots \vee b_{n}$ со свойством $\llbracket \mathbb{C}^{\sim} \models \alpha_{k} \rrbracket=b_{k}, \forall k$. Другими словами, $\llbracket \mathbb{C}^{\sim} \models \alpha_{k} \rrbracket=\top$ уже в смысле булевых подалгебр $\mathbf{B}_{k}=\left\{b \in \mathbf{B}: b \leqslant b_{k}\right\}$. В упомянутых примерах оказывается возможным склеивание результатов, полученных для отдельных $k$, давая в результате искомую теорему переноса.

\section{4f. Нестандартное представление стандартного гомоморфизма} групп. Понятно, что ключевым моментом схемы переноса п. 4c является построение нестандартного представления заданной математической структуры $\mathfrak{A}$ в виде $(\mathbb{X} \sim)^{\wedge}$ для подходящей более простой структуры $\mathbb{X}$. Здесь имеется ряд интересных задач, рассмотренных в [59], [32], из которых мы кратко обсудим одну.

Предположим, что $\mathbb{A}$ и $\mathbb{X}$ - полные метрические пространства, а $\mathbf{B}$ - полная булева алгебра со стоуновым пространством $\mathscr{S}$. Предположим, что каждому $a \in \mathbb{A}$ сопоставлена функция $f_{a}: S_{a} \rightarrow \mathbb{X}$, определенная и непрерывная на плотном $\mathbf{G}_{\delta}$-множестве $S_{a} \subseteq \mathscr{S}$. Другими словами, $f_{a} \in \mathbf{C}^{\prime \prime}(\mathscr{S}, \mathbb{X})$. По теореме 4.2, каждому $a \in \mathbb{A}$ сопоставляется $p_{a}=p_{\mathbf{f}_{a}} \in\left(\mathbb{X}^{\sim}\right)^{\wedge}$ так, что

$$
\llbracket p_{a} \in F^{\sim} \rrbracket \geqslant b \Leftrightarrow f_{a} "\left(\mathscr{S}_{p} \cap S_{a}\right) \subseteq F \quad-\text { для всех } b \in \mathbf{B} \text { и } F \in \mathscr{F} .
$$

(Как и выше, $\mathscr{F}$ - все непустые замкнутые множества $F \subseteq \mathbb{X}$.) Итак, $a \mapsto p_{a}$ отображает $\mathbb{A}$ в $\left(\mathbb{X}^{\sim}\right)^{\wedge}$. В этом случае существует $t \in \mathbf{V}^{(\mathbf{B})}$ такое, что с оценкой $\top$ в $\mathbf{V}^{(\mathbf{B})}$ выполнено " $t-$ функция из $\mathbb{A}^{\vee}$ в $\mathbb{X}^{\sim}$ и $t\left(a^{\vee}\right)=p_{a}$ " для каждого $a \in \mathbb{A}$.

Когда $t$ продолжается в $\mathbf{V}^{(\mathbf{B})}$ (с оценкой $丁$ ) до непрерывной $t^{\prime}: \mathbb{A}^{\sim} \rightarrow \mathbb{X}^{\sim}$ ? Эта задача решается в общем виде, но мы расссмотрим ее при следующих упрощающих предположениях.

1) $\mathbb{A}$ является непрерывной абелевой группой, а метрика $\rho$ пространства $\mathbb{X}$ инвариантна: $\rho(a, b)=\rho(a+c, b+c)$ для всех $a, b, c \in A$.

$2) \mathbb{X}=\mathbb{C}-$ комплексные числа. Вследствие $\sigma$-компактности, множества $S_{a}=\operatorname{dom} f_{a}$ считаются открытыми плотными в $\mathscr{S}$, так что $f_{a} \in \mathbf{C}^{\prime}(\mathscr{S}, \mathbb{X})$ (см. замечание 2) в конце п. $4 \mathrm{~d}$ ).

3) Отображение $a \mapsto f_{a}$ является гомоморфизмом в том смысле, что для любой пары $a, b \in \mathbb{A}$ равенство $f_{a}(s)+f_{b}(s)=f_{a+b}(s)$ выполнено для всех $s$ из некоторого плотного открытого множества $D=D_{a b} \subseteq \mathscr{S}$.

Определим $\|a\|=\rho(\mathbf{0}, a)$, где $\mathbf{0}-$ нейтральный элемент группы $\mathbb{A}$, так что $\rho(a, b)=\|a-b\|$. Через $|z|$ обозначается модуль $z \in \mathbb{C}$.

Tеорема 4.6. В этих обозначениях и условиях необходимым и достаточным условием для существования $t^{\prime} \in \mathbf{V}^{(\mathbf{B})}$ такого, что с оценкой $\top$ в $\mathbf{V}^{(\mathbf{B})}$ выполнено " $t$ ' - непрерывный гомоморфизм $\mathbb{A}^{\sim} \rightarrow \mathbb{C}^{\sim}$ и продолжение $t$ ", является условие: 
для всякого $k \geqslant 1$ найдется функиия $g_{k}: D_{k} \rightarrow \mathbb{N}$, определенная и непрерывная на некотором открытом плотном множестве $D_{k} \subseteq \mathscr{S}$ и такал, что для любьх $s \in \mathscr{S}$ u $a \in \mathbb{A}$ uз $s \in S_{a} \cap D_{k} u\|a\|<g_{k}(s)^{-1}$ следует $\left|f_{a}(s)\right|<k^{-1}$.

Это условие, очевидно, слабее равномерной по $s$ сходимости $f_{a}(s)$ к 0 при $a \rightarrow \mathbf{0}$ (которая получается при $g_{k}$, равных подходящим константам), но сильнее поточечной сходимости "почти везде", т.е. на некотором открытом плотном множестве.

ДокАЗАтЕльство. Достаточность. Оставим читателю несложное доказательство того, что " $t$ - гомоморфизм из $\mathbb{A}^{\vee}$ в $\mathbb{C}^{\sim}$ в $\mathbf{V}^{(\mathbf{B})}$ с оценкой Т. Из-за свойства инвариантности метрики $\mathbb{A}$ (которое переносится и в $\mathbf{V}^{(\mathbf{B})}$ ), задача сводится к доказательству “непрерывности $t$ в $\mathbf{0}$ " в $\mathbf{V}^{(\mathbf{B})}$, которое мы сейчас приведем.

Фиксируем $k \geqslant 1$. По теореме $4.2(\mathrm{ii})$, функция $g_{k}$ из условия теоремы порождает элемент $\nu_{k}=\mathbf{p}_{g_{k}} \in \mathbf{V}^{(\mathbf{B})}$ такой, что $\llbracket \nu_{k} \in \mathbb{N}^{\vee} \rrbracket=\top$ и $\mathbf{f}_{\nu_{k}} \equiv g_{k}$. Так что имеем для всех $b \in \mathbb{B}$ и $n \in \mathbb{N}$

$$
\llbracket \nu_{k}=n \rrbracket \geqslant b \Leftrightarrow \forall s \in \mathscr{S}_{b} \cap D_{k}\left(g_{k}(s)=n\right) .
$$

Утверждаем, что $\llbracket \forall a \in \mathbb{A}^{\vee}\left(\|a\|<\nu_{k}^{-1} \Rightarrow\left|p_{a}\right|<\left(k^{\vee}\right)^{-1}\right) \rrbracket=\top$. Пусть, напротив, $a \in \mathbb{A}$ таково, что $\llbracket\left\|a^{\vee}\right\|<\nu_{k}^{-1} \wedge\left|p_{a}\right| \geqslant\left(k^{\vee}\right)^{-1} \rrbracket=b>\perp$. Найдется $n$ такое, что $c=b \cap \llbracket \nu_{k}=n \rrbracket>\perp$, и тогда $\llbracket\left\|a^{\vee}\right\|<\left(n^{\vee}\right)^{-1} \wedge\left|p_{a}\right| \geqslant\left(k^{\vee}\right)^{-1} \rrbracket=c$. Тогда $g_{k}(s)=n$ для всех $s \in D_{k} \cap \mathscr{S}_{c}$ по (18), а потому $\left|f_{a}(s)\right|<k^{-1}$ для всех $s \in D_{k} \cap S_{a} \cap \mathscr{S}_{c}$ согласно условию теоремы, откуда $\llbracket\left|p_{a}\right|<\left(k^{\vee}\right)^{-1} \rrbracket \geqslant c$ по (17), что противоречит выбору $b$.

Необходимость. Предположим, что $t^{\prime} \in \mathbf{V}^{(\mathbf{B})}$ и

$\llbracket t^{\prime}$ есть непрерывный гомоморфизм $\mathbb{A}^{\sim} \rightarrow \mathbb{C}^{\sim}$ и продолжение $t \rrbracket=\top$.

$\mathrm{B}$ частности, $\llbracket t^{\prime}$ непрерывно в $\mathbf{0} \rrbracket=\top$. Поэтому для каждого $k$ найдется элемент $\nu_{k} \in \mathbf{V}^{(\mathbf{B})}$ такой, что оценка " $\nu_{k} \in \mathbb{N}^{\vee}$ и $\forall a \in \mathbb{A}^{\sim}\left(\|a\|<\nu_{k}{ }^{-1} \Rightarrow\left|t^{\prime}(a)\right|<\right.$ $\left(k^{\vee}\right)^{-1}$ )" равна $\top$. Остается взять $g_{k}=\mathbf{f}_{\nu_{k}}$ (теорема 4.2(i)). Теорема 4.6 доказана.

В статье [31] предложен иной подход к рассматриваемой задаче. Оказывается, что если в качестве $\mathbf{B}$ взять полную булеву алгебру проекторов в подходящем банаховом пространстве, то условие интегрируемости по Бохнеру становится достаточным для непрерывной продолжаемости в духе теоремы 4.6 (теорема 16 в [31], и ранее аналогичный результат в [58]). В качестве $\mathfrak{A}$ берется локально компактная абелева группа.

Исторические и библиографические замечания к $\$ 4$. Основы форсинга были заложены в исследованиях П. Дж. Коэна [35] по континуум-гипотезе. Систематическое изложение обобщенного варианта форсинга в форме булевозначного универсума (включая теорему 4.1) впервые появилось, вероятно, в [34], [50], где отмечено, что это обобщение основано на более ранних работах Д. Скотта, Р. М. Соловея [61], и П. Вопенки [62], см. также [63], [64]. В этих публикациях [34], [50] появились обозначения $x^{\vee}$ и $X^{\wedge}$, в книге [29] $X^{\wedge}$ переобозначено через $X \downarrow$ ("спуск" $X)$. 
Теорема 4.2 вместе с модификациями для компактных и $\sigma$-компактных пространств (и даже в более общей форме для равномерных по Бурбаки [60], а не метрических пространств) появилась в работах В. А. Любецкого и Е. И. Гордона [58], [65], [59] (а некоторые предварительные идеи в [66]). Попытки усилить результат до непрерывных функций на открытых плотных множествах для случая полных равномерных топологических пространств требуют дальнейших усилий. В некоторой мере предшественником этих результатов является представление вещественных чисел в случайных, генерических (по Коэну) и некоторых других моделях теории множеств как значений непрерывных функций от расширяющего числа.

Определение $X^{\sim}$ для полных топологических пространств и другие связанные с $X^{\sim}$ понятия и результаты с полными доказательствами получены в [58].

О хорновых формулах см., например, [39; 6.2]. Можно усмотреть некоторое сходство леммы 4.4 с теоремой 6.2 .2 в [39; 6.2$]$ об устойчивости хорновых формул.

Теорема 4.6 доказана авторами.

\section{Список литературы}

[1] А. Робинсон, Введение в теорию моделей и метаматематику алгебры, Наука, М., 1967; пер. с англ.: А. Robinson, Introduction to model theory and to the metamathematics of algebra, North-Holland, Amsterdam, 1963.

[2] В. А. Успенский, Нестандартный, или неархимедов анализ, Новое в жизни, науке, технике. Сер. "Математика, кибернетика", 8, Знание, М., 1983.

[3] В. А. Успенский, Что такое нестандартный анализ?, Наука, М., 1987.

[4] Е.И. Гордон, А.Г. Кусраев, С. С. Кутателадзе, Инфинитезимальный анализ, ч. 1, 2, Изд-во Ин-та матем. СО РАН, Новосибирск, 2001; англ. пер.: Е. I. Gordon, A. G. Kusraev, S. S. Kutateladze, Infinitesimal analysis, Math. Appl., 544, Kluwer Acad. Publ., Dordrecht, 2002.

[5] М. Девис, Прикладной нестандартный анализ, Мир, М., 1980; пер. с англ.: М. Davis, Applied nonstandard analysis, Pure Appl. Math., Wiley-Intersci., New York-London-Sydney, 1977.

[6] А. К. Звонкин, М. А. Шубин, "Нестандартный анализ и сингулярные возмущения обыкновенных дифференциальных уравнений”, УМН, 39:2 (1984), 77-127; англ. пер.: A. K. Zvonkin, M. A. Shubin, "Non-standard analysis and singular perturbations of ordinary differential equations", Russian Math. Surveys, 39:2 (1984), 69-131.

[7] P. A. Loeb, M. Wolff (eds.), Nonstandard analysis for the working mathematician, Math. Appl., 510, Kluwer Acad. Publ., Dordrecht, 2000.

[8] E. Nelson, "Internal set theory: A new approach to nonstandard analysis", Bull. Amer. Math. Soc., 83:6 (1977), 1165-1198.

[9] E. Nelson, "The syntax of nonstandard analysis", Ann. Pure Appl. Logic, 38 (1988), 123-134.

[10] K. Hrbáček, "Axiomatic foundations for nonstandard analysis", Fund. Math., 98:1 (1978), 1-19.

[11] K. Hrbáček, "Nonstandard set theory", Amer. Math. Monthly, 86:8 (1979), 659-677.

[12] T. Kawai, "Axiom systems of nonstandard set theory", Logic Symposia (Hakone, 1979/1980), Lecture Notes in Math., 891, Springer, Berlin, 1981, 57-65.

[13] T. Kawai, "Nonstandard analysis by axiomatic methods", Southeast Asia Conference on Logic (Singapore, 1981), Stud. Logic Found. Math., 111, North-Holland, Amsterdam, 1983, 55-76. 
[14] В.Г. Кановей, "Неразрешимые гипотезы в теории внутренних множеств Эдварда Нельсона", УМH, 46:6 (1991), 3-50; англ. пер.: V. G. Kanovei, "Undecidable hypotheses in Edward Nelson's internal set theory", Russian Math. Surveys, 46:6 (1991), 1-54.

[15] P. V. Andreev, E. I. Gordon, "An axiomatics for nonstandard set theory, based on von Neumann-Bernays-Gödel theory", J. Symbolic Logic, 66:3 (2001), 1321-1341.

[16] P. Andreev, K. Hrbacek, "Standard sets in nonstandard set theory", J. Symbolic Logic, 69:1 (2004), 165-182.

[17] D. Ballard, K. Hrbáček, "Standard foundations for nonstandard analysis", J. Symbolic Logic, 57:2 (1992), 741-748.

[18] K. Hrbáček, "Realism, nonstandard set theory, and large cardinals", Ann. Pure Appl. Logic, 109:1-2 (2001), 15-48.

[19] K. Hrbáček, "Internally iterated ultrapowers", Nonstandard models of arithmetic and set theory, Proceedings of the Special Session on Nonstandard Methods (Baltimore, 2003), Contemp. Math., 361, eds. A. Enayat, R. Kossak, Amer. Math. Soc., Providence, RI, 2004, 87-120.

[20] К. Хрбачек, "Заметки о нестандартной теории классов", Фундам. и прикл. матем., 11:5 (2005), 233-255.

[21] V. Kanovei, M. Reeken, "Internal approach to external sets and universes. I: Bounded set theory", Studia Logica, 55:2 (1995), 229-257; "II: External universes over the universe of bounded set theory", 55:3 (1995), 347-376; "III: Partially saturated universes", 56:3 (1996), 293-322.

[22] V. Kanovei, M. Reeken, "Mathematics in a nonstandard world. I, II", Math. Japon., 45:2 (1997), 369-408; 45:3, 555-571.

[23] V. Kanovei, M. Reeken, "Isomorphism property in nonstandard extensions of the ZFC universe", Ann. Pure Appl. Logic, 88:1 (1997), 1-25.

[24] V. Kanovei, S. Shelah, "A definable nonstandard model of the reals", J. Symbolic Logic, 69:1 (2004), 159-164.

[25] V. Kanovei, M. Reeken, Nonstandard analysis, axiomatically, Springer Monogr. Math., Springer-Verlag, Berlin, 2004.

[26] A.S. Kechris, Classical descriptive set theory, Grad. Texts in Math., 156, SpringerVerlag, Berlin, 1995.

[27] В. Г. Кановей, В. А. Любецкий, "О некоторых классических проблемах дескриптивной теории множеств", УМH, 58:5 (2003), 3-88; англ. пер.: V. G. Kanovei, "On some classical problems of descriptive set theory", Russian Math. Surveys, 58:5 (2003), 839-927.

[28] В. Г. Кановей, В. А. Успенский, “О единственности нестандартных расширений”, Вестн. МГУ. Сер. 1. Матем., мех., 5 (2006), 3-10.

[29] А.Г. Кусраев, С. С. Кутателадзе, Нестандартные методы анализа, Наука, Новосибирск, 1990; англ. пер.: А. G. Kusraev, S. S. Kutateladze, Nonstandard methods of analysis, Math. Appl., 291, Kluwer Acad. Publ., Dordrecht, 1994.

[30] А.Г. Кусраев, С. С. Кутателадзе, Булевозначный анализ, Изд-во Ин-та матем. CO PAН, Новосибирск, 1999; англ. пер.: А. G. Kusraev, S. S. Kutateladze, Boolean valued analysis, Kluwer Acad. Publ., Dordrecht, 1999.

[31] В. А. Любецкий, "Некоторые применения теории топосов к изучению алгебраических систем", Дополнение к кн.: П. Т. Джонстон, Теория топосов, Наука, М., 1986, 376-433.

[32] В.А. Любецкий, "Оценки и пучки. О некоторых вопросах нестандартного анализа", УМН, 44:4 (1989), 99-153; англ. пер.: V. A. Lyubetskii, "Valuations and sheaves. On some questions of non-standard analysis", Russian Math. Surveys, 44:4 (1989), 37-112. 
[33] В. А. Любецкий, “Теоремы переноса и алгебра модальных операторов”, Алгебра и логика, 36:3 (1997), 282-303; англ. пер.: V. A. Lyubetskii, "Transfer theorems and the algebra of modal operators", Algebra Logic, 36:3 (1997), 169-181.

[34] Т. Йех, Теория множестө и метод форсинга, Мир, М., 1973; пер. с англ.: T. J. Jech, Lectures in set theory, with particular emphasis on the method of forcing, Lecture Notes in Math., 217, Springer-Verlag, Berlin-New York, 1971.

[35] П. Дж. Коэн, Теория множеств и континуум-гипотеза, Мир, М., 1969; пер. с англ.: P. J. Cohen, Set theory and the continuum hypothesis, W. A. Benjamin, Inc., New York-Amsterdam, 1966.

[36] А.А. Френкель, И. Бар-Хиллел, Основания теории множеств, Мир, М., 1966; пер. с англ.: А. A. Fraenkel, Y. Bar-Hillel, Foundations of set theory, North-Holland, Amsterdam, 1958.

[37] T. Jech, Set theory, Pure Appl. Math., Academic Press, New York-London, 1978.

[38] K. Kunen, Set theory. An introduction to independence proofs, Stud. Logic Found. Math., 102, North-Holland, Amsterdam-New York, 1980.

[39] C. C. Chang, H. J. Keisler, Model theory, 3rd ed., Stud. Logic Found. Math., 73, North-Holland, Amsterdam, 1992; рус. пер. 1-го изд.: Г. Кейслер, Ч. Ч. Чэн, Теория моделей, Мир., М., 1977.

[40] R. Goldblatt, Lectures on the hyperreals. An introduction to nonstandard analysis, Grad. Texts in Math., 188, Springer-Verlag, New York, 1998.

[41] C. W. Henson, "Foundations of nonstandard analysis. A gentle introduction to nonstandard extension", Nonstandard analysis: theory and applications (Edinburgh, 1996), NATO Adv. Sci. Inst. Ser. C: Math. Phys. Sci., 493, Kluwer Acad. Publ., Dordrecht, 1997, 1-49.

[42] C. W. Henson, H. J. Keisler, "On the strength of nonstandard analysis", J. Symbolic Logic, 51:2 (1986), 377-386.

[43] V. Kanovei, M. Reeken, "Borel and countably determined reducibility in nonstandard domain", Monatsh. Math., 140:3 (2003), 197-231.

[44] H. J. Keisler, "The hyperreal line", Real numbers, generalizations of the reals, and theories of continua, Synth. Libr., 242, Kluwer Acad. Publ., Dordrecht, 1994, $207-$ 237.

[45] T. Lindstrøm, "An invitation to nonstandard analysis", Nonstandard analysis and its application (Hull, 1986), London Math. Soc. Stud. Texts, 10, Cambridge Univ. Press, Cambridge, 1988, 1-105.

[46] P. A. Loeb, "An introduction to nonstandard analysis", Nonstandard analysis for the working mathematician, Math. Appl., 510, Kluwer Acad. Publ., Dordrecht, 2000, $1-95$.

[47] В. Г. Кановей, "О корректности эйлерова метода разложения синуса в бесконечное произведение", УМH, 43:4 (1988), 57-81; англ. пер.: V. G. Kanovei, "The correctness of Euler's method for the factorization of the sine function into an infinite product", Russian Math. Surveys, 43:4 (1988), 65-94.

[48] Дж. Шенфилд, Математическая логика, Наука, М., 1975; пер. с англ.: J. R. Shoenfield, Mathematical logic, Addison-Wesley, London, 1967.

[49] U. Felgner, "Comparison of the axioms of local and universal choice", Fund. Math., 71:1 (1971), 43-62.

[50] R. M. Solovay, S. Tennenbaum, "Iterated Cohen extensions and Souslin's problem", Ann. of Math. (2), 94:2 (1971), 201-245.

[51] P. Vopĕnka, P. Hájek, The theory of semisets, Stud. Logic Found. Math., 70, NorthHolland, Amsterdam-London, 1972.

[52] P. Vopĕnka, Mathematics in the alternative set theory, Teubner, Leipzig, 1979. 
[53] Х. Ван, Р. Мак-Нотон, Аксиоматические системы теории множеств, ИЛ, М., 1963; пер. с фр.: H. Wang, R. McNaughton, Les systèmes axiomatiques de la théorie des ensembles, Gauthier-Villars, Paris, 1953.

[54] К. Гёдель, “Совместимость аксиомы выбора и обобщенной континуум-гипотезы с аксиомами теории множеств", УМH, 3:1 (1948), 96-149; пер. с англ.: K. Gödel, The consistency of the continuum hypothesis, Ann. of Math. Stud., 3, Princeton Univ. Press, Princeton, NJ, 1940.

[55] Дж. Л. Келли, Общая топология, Наука, M., 1968; пер. с англ.: J.L. Kelley, General topology, Van Nostrand, Toronto, 1955.

[56] K. Devlin, The joy of sets. Fundamentals of contemporary set theory, Undergrad. Texts Math., Springer-Verlag, New York, 1993.

[57] Е.И. Гордон, "Вещественные числа в булевозначных моделях теории множеств и $K$-пространства", Докл. АН СССР, 237:4 (1977), 773-775; англ. пер.: E. I. Gordon, "Real numbers in Boolean-valued models of set theory, and $K$-spaces", Soviet Math. Dokl., 18:6 (1977-1978), 1481-1484.

[58] Е.И. Гордон, В.А. Любецкий, Булевозначные расширения равномерных струкmур, І, Деп. в ВИНИТИ, 711-80, М., 1980.

[59] В.А.Любецкий, Е. И. Гордон, "Булевы расширения равномерных структур", Исследования по неклассическим логикам и формальным системам, Наука, М., $1983,82-153$.

[60] Н. Бурбаки, Общая топология, основные структуры, Наука, М., 1968; пер. c фp.: N. Bourbaki, Éléments de mathématique. Première partie. (Fascicule II). Livre III: Topologie générale. Chapitre 1: Structures topologiques. Chapitre 2: Structures uniformes, Troisiéme édition entiérement refondue. Actualités Sci. Indust., 1142, Hermann, Paris, 1961.

[61] R. M. Solovay, "A model of set-theory in which every set of reals is Lebesgue measurable", Ann. of Math. (2), 92:1 (1970), 1-56.

[62] P. Vopĕnka, "General theory of $\nabla$-models", Comment. Math. Univ. Carolin., 8 (1967), 145-170.

[63] J. L. Bell, Boolean-valued models and independence proofs in set theory, Oxford Logic Guides, Clarendon Press, Oxford, 1977.

[64] J.R. Shoenfield, "Unramified forcing", Axiomatic set theory (Univ. California, Los Angeles, Calif., 1967), Proc. Sympos. Pure Math., 13, part 1, 1971, 357-381.

[65] Е. И. Гордон, В. А. Любецкий, "Некоторые применения нестандартного анализа в теории булевозначных мер", Докл. АН CCCP, 256:5 (1981), 1037-1041; англ. пер.: E.I. Gordon, V.A. Lyubetskij, "Some applications of nonstandard analysis in the theory of Boolean-valued measures", Soviet Math. Dokl., 23 (1981), 142-146.

[66] В. А. Любецкий, "Булевозначные расширения структур", Математические методы решения инженерных задач, Академия Дзержинского, М., 1979, 67-81.

\section{В. Г. Кановей}

Институт проблем передачи информации

E-mail: kanovei@mccme.ru

\section{В. А. Любецкий}

Институт проблем передачи информации

E-mail: lyubetsk@ippi.ru
Поступила в редакцию 15.12 .2006 